\title{
Catalytic Enantioselective Synthesis of Flavanones and Chromanones
}

\author{
Margaret M. Biddle, Michael Lin and Karl A. Scheidt** \\ Department of Chemistry, Northwestern University, \\ 2145 Sheridan Road, Evanston, Illinois 60208

\section{Supporting Information}

Table of Contents

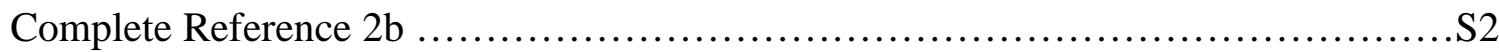

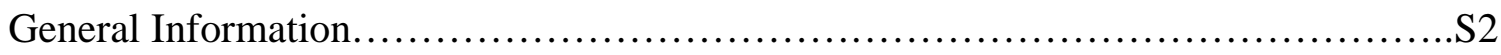

General Procedure to Access tert-Butylketoesters................................ 2

General Procedure to Access Alkene..........................................S3

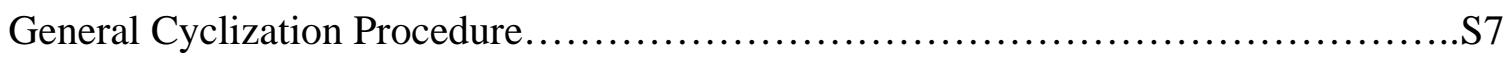

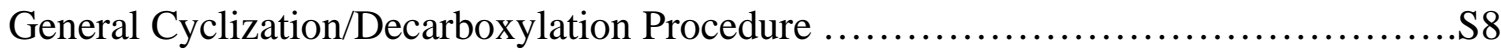

One-Pot Knoevenagel/Cyclization........................................... 11

Selected NMR Spectra.................................................S13

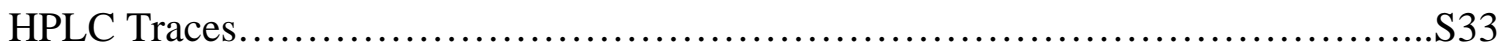

X-Ray Crystal structure for (E)-tert-butyl 3-(4-bromophenyl)-2-(2-

hydroxyphenylcarbonyl)prop-2-enoate. 
Complete Reference 2b: Tan, Q. et al.; Blizzard, T. A.; Jerry, D.; Morgan, I.; Birzin, E. T.; Chan, W.; Yang, Y. T.; Pai, L.-Y.; Hayes, E. C.; DaSilva, C. A.; Warrier, S.; Yudkovitz, J.; Wilkinson, H. A.; Sharma, N.; Fitzgerald, P. M. D.; Li, S.; Colwell, L.; Fisher, J. E.; Adamski, S.; Reszka, A. A.; Kimmel, D.; DiNinno, F.; Rohrer, S. P.; Freedman, L. P.; Schaeffer, J. M.; Hammond, M. L. Bioorg. Med. Chem. Lett. 2005, 15, 1675-1681.

\section{General Information}

All reactions were carried out under a nitrogen atmosphere in flame-dried glassware with magnetic stirring. Toluene was purified by passage through a bed of activated alumina. ${ }^{1}$ Reagents were purified prior to use unless otherwise stated following the guidelines of Perrin and Armarego. ${ }^{2}$ Purification of reaction products was carried out by flash chromatography using EM Reagent silica gel 60 (230-400 mesh). Analytical thin layer chromatography was performed on EM Reagent $0.25 \mathrm{~mm}$ silica gel $60-\mathrm{F}$ plates. Visualization was accomplished with UV light and ceric ammonium nitrate stain followed by heating. Infrared spectra were recorded on a Perkin Elmer 1600 series FT-IR spectrometer. ${ }^{1} \mathrm{H}-\mathrm{NMR}$ spectra were recorded on a Varian Inova $500(500 \mathrm{MHz})$ spectrometer and are reported in ppm using solvent as an internal standard $\left(\mathrm{CDCl}_{3}\right.$ at 7.26 $\mathrm{ppm}$ ). Data are reported as ( $\mathrm{ap}=$ apparent, $\mathrm{s}=$ singlet, $\mathrm{d}=$ doublet, $\mathrm{t}=$ triplet, $\mathrm{q}=$ quartet, $\mathrm{m}=$ multiplet, $\mathrm{b}=$ broad; coupling constant(s) in $\mathrm{Hz}$; integration. Proton-decoupled ${ }^{13} \mathrm{C}$ NMR spectra were recorded on a Varian Inova $500(125 \mathrm{MHz})$ spectrometer and are reported in $\mathrm{ppm}$ using solvent as an internal standard $\left(\mathrm{CDCl}_{3}\right.$ at $\left.77.0 \mathrm{ppm}\right)$. Mass spectra data were obtained on a Varian 1200 Quadrupole Mass Spectrometer and Micromass Quadro II Spectrometer.

Thiourea catalyst I was prepared according to the procedure of Soós. ${ }^{3}$ Thiourea catalyst II was prepared according to the procedure of Takemoto. ${ }^{4}$ Thiourea catalyst III was prepared according to the procedure of Hiemstra. ${ }^{5}$

\section{General Procedure to Access tert-Butyl 3-(2-hydroxyphenyl)-3-oxopropanoates (18)}

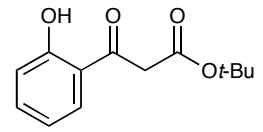

tert-butyl 3-(2-hydroxyphenyl)-3-oxopropanoate (18): To a $\mathrm{N}_{2}$ purged $250 \mathrm{~mL} \mathrm{RBF}$ is added $30 \mathrm{~mL}$ of THF and diisopropylamine $(12.0 \mathrm{~mL}$, $86 \mathrm{mmol})$. The solution is cooled to $-78^{\circ} \mathrm{C}$ and $n \mathrm{BuLi}(50 \mathrm{~mL}, 1.6 \mathrm{M})$ is added and the solution is warmed to $0{ }^{\circ} \mathrm{C}$ for $45 \mathrm{~min}$. The solution is cooled to $-78{ }^{\circ} \mathrm{C}$ and $t$-butyl acetate $(7.1 \mathrm{~mL}, 53 \mathrm{mmol})$ in $12 \mathrm{~mL}$ THF is added dropwise over 10 minutes. After $90 \mathrm{~min}$, ethyl salicylate $(2.2 \mathrm{~mL}, 15 \mathrm{mmol})$ in $15 \mathrm{~mL}$ of THF is added. The solution is allowed to warm to RT overnight and quenched with $90 \mathrm{~mL}$ of aq. $\mathrm{NH}_{4} \mathrm{Cl}$ (sat.), extracted with EtOAc $(2 \times 25 \mathrm{~mL})$, washed brine $(40 \mathrm{~mL})$, dried $\mathrm{Na}_{2} \mathrm{SO}_{4}$, filtered,

1. Pangborn, A. B.; Giardello, M. A.; Grubbs, R. H.; Rosen, R. K.; Timmers, F. J. Organometal. 1996, 15, 1518-1520.

2. Perrin, D. D. and Armarego, W. L. Purification of Laboratory Chemicals; 3rd Ed., Pergamon Press, Oxford. 1988.

3. Vakulya, B.; Varga, S.; Csampai, A.; Soos, T. Org. Lett. 2005, 7, 1967-1969.

4. Okino, T.; Hoashi, Y.; Takemoto, Y. J. Am. Chem. Soc. 2003, 125, 12672-12673.

5. Marcelli, T.; van der Haas, R. N. S.; van Maarseveen, J. H.; Hiemstra, H. Angew. Chem., Int. Ed. 2006, $45,929-931$. 
concentrated in vacuo. Purified via column chromatography (silica gel, 10\% EtOAc/hex) to give $2.65 \mathrm{~g}$ of pale yellow oil (75\% yield). IR (film) 2980.2; 2934.9; 1733.6; 1643.7; 1146.6; $757.0 \mathrm{~cm}^{-1} ;{ }^{1} \mathrm{H}$ NMR $\left(500 \mathrm{MHz}, \mathrm{CDCl}_{3}\right) \delta 11.93(\mathrm{~s}, 1 \mathrm{H}) ; 7.68(\mathrm{~d}, J=7.9 \mathrm{~Hz}$, $1 \mathrm{H}) ; 7.51(\mathrm{t}, J=7.3,1 \mathrm{H}) ; 7.01(\mathrm{~d}, J=8.2 \mathrm{~Hz}, 1 \mathrm{H}) ; 6.93(\mathrm{t}, J=7.3 \mathrm{~Hz}, 1 \mathrm{H}) ; 3.93(\mathrm{~s}, 2 \mathrm{H})$; 1.47 (s, 9H); ${ }^{13} \mathrm{C}$ NMR $\left(125 \mathrm{MHz}, \mathrm{CDCl}_{3}\right) \delta 198.7,165.8,162.3,136.6,130.3,118.8$, 118.7, 118.3, 82.1, 46.9, 27.6; LRMS (electrospray): Mass calculated for $\mathrm{C}_{13} \mathrm{H}_{16} \mathrm{O}_{4}$, [M] 236.10. Found, $[\mathrm{M}+23]^{+} 259.5$.

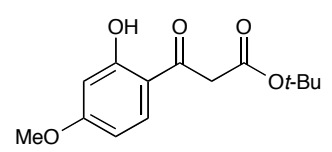

tert-butyl 3-(2-hydroxy-4-methoxyphenyl)-3-oxopropanoate: Prepared according to above procedure using diisopropylamine $(8.0$ $\mathrm{mL}, 58 \mathrm{mmol}), \mathrm{nBuLi}(34 \mathrm{~mL}, 1.6 \mathrm{M})$, tert-butyl acetate $(4.7 \mathrm{~mL}, 35$ mmol), methyl 4-methoxysalicylate $(1.83 \mathrm{~g}, 10 \mathrm{mmol})$ yielding $2.04 \mathrm{~g}(76 \%)$ as white needles. $\mathrm{mp}=40-42{ }^{\circ} \mathrm{C}$; IR (film) $2978.7 ; 2936.4 ; 1731.9 ; 1633.5 ; 1357.0 ; 1129.3 \mathrm{~cm}^{-1}$; ${ }^{1} \mathrm{H}$ NMR $\left(500 \mathrm{MHz}, \mathrm{CDCl}_{3}\right) \delta 12.42(\mathrm{~s}, 1 \mathrm{H}) ; 7.57(\mathrm{~d}, J=9.0 \mathrm{~Hz}, 1 \mathrm{H}) ; 6.45(\mathrm{~d}, J=9.0$, $1 \mathrm{H}) ; 6.44(\mathrm{~s}, 1 \mathrm{H}) ; 3.85(\mathrm{~s}, 3 \mathrm{H}) ; 3.84(\mathrm{~s}, 2 \mathrm{H}) ; 1.46(\mathrm{~s}, 9 \mathrm{H}) ;{ }^{13} \mathrm{C} \mathrm{NMR}\left(125 \mathrm{MHz}, \mathrm{CDCl}_{3}\right) \delta$ 197.1, 166.7, 166.6, 165.9, 132.3, 113.5, 108.3, 101.2, 82.5, 55.9, 47.2, 28.2; LRMS (electrospray): Mass calculated for $\mathrm{C}_{14} \mathrm{H}_{18} \mathrm{O}_{5},[\mathrm{M}]^{+}, 266.12$. Found $[\mathrm{M}+23]^{+}, 289.5$.

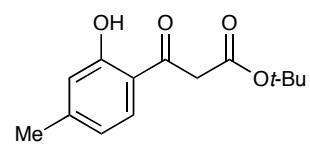

tert-butyl

3-(2-hydroxy-4-methylphenyl)-3-oxopropanoate:

Prepared according to above procedure using diisopropylamine (3.8 $\mathrm{mL}, 28 \mathrm{mmol}), \mathrm{nBuLi}(21 \mathrm{~mL}, 1.2 \mathrm{M})$, tert-butyl acetate $(2.1 \mathrm{~mL}, 16$ mmol), 2-hydroxy -4-methylbenzoyl chloride (5 mmol, prepared from $760 \mathrm{mg}$ 4-methylsalicylic acid and $\mathrm{SOCl}_{2}, 1.2 \mathrm{~mL}$, heated to $47^{\circ} \mathrm{C}$ in $12 \mathrm{~mL}$ toluene for $4 \mathrm{~h}$ ) yielding 327 $\mathrm{mg}(26 \%)$ as white needles. $\mathrm{mp}=88-90{ }^{\circ} \mathrm{C}$; IR (film) $1733.8 ; 1652.6 ; 1145.0 \mathrm{~cm}^{-1} ;{ }^{1} \mathrm{H}$ NMR (500 MHz, CDCl $) \delta 11.95(\mathrm{~s}, 1 \mathrm{H}) ; 7.54(\mathrm{~d}, J=8.2 \mathrm{~Hz}, 1 \mathrm{H}) ; 6.81(\mathrm{~s}, 1 \mathrm{H}) ; 6.72(\mathrm{~d}$, $J=8.2,1 \mathrm{H}) ; 3.88(\mathrm{~s}, 2 \mathrm{H}) ; 2.36(\mathrm{~s}, 3 \mathrm{H}) ; 1.45(\mathrm{~s}, 9 \mathrm{H}) ;{ }^{13} \mathrm{C} \mathrm{NMR}\left(125 \mathrm{MHz}, \mathrm{CDCl}_{3}\right) \delta$ 198.5, 166.5, 163.0, 148.9, 130.5, 120.7, 118.8, 117.2, 82.6, 47.4, 28.1, 22.2; LRMS (electrospray): Mass calculated for $\mathrm{C}_{14} \mathrm{H}_{18} \mathrm{O}_{4},[\mathrm{M}]^{+}, 250.12$. Found $[\mathrm{M}+23]^{+}, 272.9$.

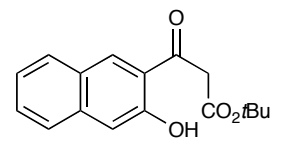

tert-butyl 3-(3-hydroxynaphthalen-2-yl)-3-oxopropanoate: Prepared according to above procedure using diisopropylamine $(2.0 \mathrm{~mL}, 15$ $\mathrm{mmol}), \mathrm{nBuLi}(11.4 \mathrm{~mL}, 1.2 \mathrm{M})$, tert-butyl acetate $(3.4 \mathrm{~mL}, 12 \mathrm{mmol})$, 3-hydroxynaphthalene-2-carbonyl chloride $(3.9 \mathrm{mmol}$, prepared from $730 \mathrm{mg}$ of 3hydroxy-2-naphthoic acid and $\mathrm{SOCl}_{2}, 0.9 \mathrm{~mL}$, heated to $40{ }^{\circ} \mathrm{C}$ in $9 \mathrm{~mL}$ toluene for $4 \mathrm{~h}$ ) yielding $666 \mathrm{mg}(57 \%)$ as yellow needles. $\mathrm{mp}=80-84{ }^{\circ} \mathrm{C}$; IR (film) $2981.9 ; 2919.0$; $1732.1 ; 1642.5 ; 1261.4 ; 1147.6 \mathrm{~cm}^{-1} ;{ }^{1} \mathrm{H}$ NMR $\left(500 \mathrm{MHz}, \mathrm{CDCl}_{3}\right) \delta 11.17(\mathrm{~s}, 1 \mathrm{H}) ; 8.29$ $(\mathrm{s}, 1 \mathrm{H}) ; 7.78(\mathrm{~d}, J=8.0 \mathrm{~Hz}, 1 \mathrm{H}) ; 7.65(\mathrm{~d}, J=8.2 \mathrm{~Hz}, 1 \mathrm{H}) ; 7.50(\mathrm{t}, J=7.0,1 \mathrm{H}) ; 7.33-7.27$ (m, 2H); 4.05 (s, 2H); 1.42 (s, 9H); ${ }^{13} \mathrm{C}$ NMR $\left(125 \mathrm{MHz}, \mathrm{CDCl}_{3}\right) \delta$ 199.3, 166.2, 157.2, 138.5, 133.7, 130.2, 129.6, 127.1, 126.5, 124.5, 120.9, 112.7, 82.9, 47.8, 28.1; LRMS (electrospray): Mass calculated for $\mathrm{C}_{17} \mathrm{H}_{18} \mathrm{O}_{4},[\mathrm{M}]^{+}, 286.12$. Found $[\mathrm{M}]^{+}, 285.8$.

Two general procedures were followed for the synthesis of alkene substrates:

General Procedure A for the preparation of alkylidene substrates: To a $25 \mathrm{~mL}$ RBF was added tert-butyl 3-(2-hydroxyphenyl)-3-oxopropanoate $\mathbf{1 8}(618 \mathrm{mg}, 2.6 \mathrm{mmol})$, benzaldehyde ( $265 \mu \mathrm{L}, 2.6 \mathrm{mmol}) 12.0 \mathrm{~mL}$ benzene, piperidine $(13 \mu \mathrm{L}, 0.13 \mathrm{mmol})$ and glacial acetic acid $(7.5 \mu \mathrm{L}, 0.13 \mathrm{mmol})$. The flask was equipped with a Dean-Stark trap 
and water condenser and heated to reflux. The reaction was allowed to cool to RT, taken up in EtOAc $(40 \mathrm{~mL})$ and washed with brine $(25 \mathrm{~mL})$. The organic layer was dried with $\mathrm{Na}_{2} \mathrm{SO}_{4}$, filtered concentrated to give a yellow oil which was recrystallized from hexanes $/ \mathrm{CH}_{2} \mathrm{Cl}_{2}$.

General Procedure B for the preparation of alkylidene substrates: To a $25 \mathrm{~mL}$ RBF was added tert-butyl 3-(2-hydroxyphenyl)-3-oxopropanoate (18) (259 mg, $1.1 \mathrm{mmol}), 2$ naphthaldehyde $(172 \mathrm{mg}, 1.1 \mathrm{mmol})$ and $\mathrm{Na}_{2} \mathrm{SO}_{4}(2.34 \mathrm{~g}, 16.5 \mathrm{mmol})$. The flask was purged with $\mathrm{N}_{2}$. Toluene $(12 \mathrm{~mL})$ and piperidinium acetate $(0.025 \mathrm{M}$ in toluene, $2.2 \mathrm{~mL})$ were added and the heterogenous mixture was stirred at RT for $7 \mathrm{~d}$. The reaction was taken up in EtOAc $(40 \mathrm{~mL})$ and washed with brine $(25 \mathrm{~mL})$. The organic layer was dried with $\mathrm{Na}_{2} \mathrm{SO}_{4}$, filtered concentrated to give a tan oil which was recrystallized from hexanes $/ \mathrm{CH}_{2} \mathrm{Cl}_{2}$.

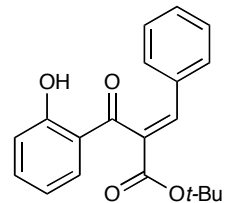

(E)-tert-butyl 2-(2-hydroxyphenylcarbonyl)-3-phenylprop-2-enoate (5): Prepared according to general procedure A using tert-butyl 3-(2hydroxyphenyl)-3-oxopropanoate (18) $(618 \mathrm{mg}, 2.6 \mathrm{mmol})$, benzaldehyde $(265 \mu \mathrm{L}, 2.6 \mathrm{mmol}), 12.0 \mathrm{~mL}$ benzene, piperidine $(13 \mu \mathrm{L}, 0.13 \mathrm{mmol})$ and glacial acetic acid $(7.5 \mu \mathrm{L}, 0.13 \mathrm{mmol})$, refluxed for $2 \mathrm{~h}$. Purified via recrystallization from hexanes $/ \mathrm{CH}_{2} \mathrm{Cl}_{2}$, yielding $708 \mathrm{mg}(83 \%)$ of $\mathbf{5}$ as clear crystals. mp $=109-110{ }^{\circ} \mathrm{C}$; IR (film) 2978.4; 1737.4; 1692.4; 1643.3; $1145.8 \mathrm{~cm}^{-1} ;{ }^{1} \mathrm{H}$ NMR (500 $\left.\mathrm{MHz}, \mathrm{CDCl}_{3}\right) \delta 11.92(\mathrm{bs}, 1 \mathrm{H}), 7.86(\mathrm{~s}, 1 \mathrm{H}), 7.55(\mathrm{~d}, J=8.0 \mathrm{~Hz}, 1 \mathrm{H}), 7.49(\mathrm{t}, J=8.4 \mathrm{~Hz})$, $1 \mathrm{H}, 7.37-7.28(\mathrm{~m}, 5 \mathrm{H}), 7.04(\mathrm{~d}, J=8.4 \mathrm{~Hz} .1 \mathrm{H}), 6.83(\mathrm{t}, J=7.3 \mathrm{~Hz}, 1 \mathrm{H}), 1.42(\mathrm{~s}, 9 \mathrm{H}) ;{ }^{13} \mathrm{C}$ NMR $\left(125 \mathrm{MHz}, \mathrm{CDCl}_{3}\right) \delta$ 201.6, 163.8, 162.8, 142.3, 137.2, 133.0, 131.9, 131.6, 130.7, 130.3, 129.1, 120.2, 119.6, 118.5, 82.8, 28.1; LRMS (electrospray): Mass calculated for $\mathrm{C}_{20} \mathrm{H}_{20} \mathrm{O}_{4},[\mathrm{M}]^{+}$, 324.13. Found $[\mathrm{M}+23]^{+}$, 347.3. An NOE difference experiment was conducted for this compound and a positive NOE was seen for the alkene proton when the tert-butyl signal was irradiated and a positive NOE was seen for the tert-butyl signal upon irradiation of the alkene proton.

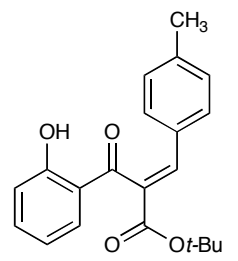

(E)-tert-butyl 2-(2-hydroxyphenylcarbonyl)-3-p-tolylprop-2-enoate: Prepared according to general procedure A using tert-butyl 3-(2hydroxyphenyl)-3-oxopropanoate (18) $(354 \mathrm{mg}, 1.5 \mathrm{mmol}), \quad p$ tolualdehyde $(150 \mu \mathrm{L}, 1.5 \mathrm{mmol}), 15.0 \mathrm{~mL}$ benzene, piperidine $(15 \mu \mathrm{L}$, $0.15 \mathrm{mmol})$ and glacial acetic acid $(8.6 \mu \mathrm{L}, 0.15 \mathrm{mmol})$, refluxed for $3 \mathrm{~h}$. Purified via recrystallization from hexanes $/ \mathrm{CH}_{2} \mathrm{Cl}_{2}$, yielding $350 \mathrm{mg}$ $(69 \%)$ of clear prisms. $\mathrm{mp}=86-89{ }^{\circ} \mathrm{C}$; IR (film) $2978.2 ; 1712.8 ; 1624.5 ; 1155.2 \mathrm{~cm}^{-1} ;{ }^{1} \mathrm{H}$ NMR (100.7 MHz, CDCl $\left.)_{3}\right) \delta 11.93(\mathrm{bs}, 1 \mathrm{H}), 7.82(\mathrm{~s}, 1 \mathrm{H}), 7.52(\mathrm{~d}, J=8.0 \mathrm{~Hz}, 1 \mathrm{H}), 7.48$ (t, $J=8.2 \mathrm{~Hz}, 1 \mathrm{H}), 7.25-7.08\left(\mathrm{AA}^{\prime} \mathrm{BB}^{\prime}, 4 \mathrm{H}\right), 7.03(\mathrm{~d}, J=8.2 \mathrm{~Hz}, 1 \mathrm{H}), 6.81(\mathrm{t}, J=7.6 \mathrm{~Hz}$, $1 \mathrm{H}), 2.30(\mathrm{~s}, 3 \mathrm{H}), 1.40(\mathrm{~s}, 9 \mathrm{H}) ;{ }^{13} \mathrm{C} \mathrm{NMR}\left(125 \mathrm{MHz}, \mathrm{CDCl}_{3}\right) \delta 202.0,163.9,162.8$, 142.3, 141.3, 137.1, 131.9, 130.4 (x2), 130.1, 129.9, 120.2, 119.5, 118.4, 82.6, 28.1, 21.6; LRMS (electrospray): Mass calculated for $\mathrm{C}_{21} \mathrm{H}_{22} \mathrm{O}_{4},[\mathrm{M}]^{+}, 338.14$. Found $[\mathrm{M}+23]^{+}$, 361.4 . 


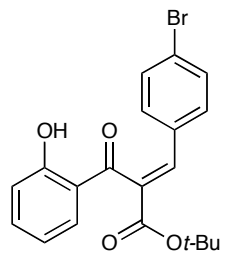

(E)-tert-butyl 3-(4-bromophenyl)-2-(2-hydroxyphenylcarbonyl)prop2-enoate : Prepared according to general procedure A using tert-butyl 3(2-hydroxyphenyl)-3-oxopropanoate (18) (289 mg, 1.2 mmol), 4bromobenzaldehyde ( $226 \mathrm{mg}, 1.2 \mathrm{mmol}$ ), $20.0 \mathrm{~mL}$ benzene, piperidine (10 $\mu \mathrm{L}, 0.1 \mathrm{mmol})$ and glacial acetic acid $(5.6 \mu \mathrm{L}, 0.1 \mathrm{mmol})$, refluxed for $3 \mathrm{~h}$. Purified via recrystallization from hexanes/ $\mathrm{CH}_{2} \mathrm{Cl}_{2}$, yielding $278 \mathrm{mg}(57 \%)$ of clear crystals. $\mathrm{mp}=98-100{ }^{\circ} \mathrm{C}$; IR (film) $2978.4 ; 1712.8 ; 1629.0 ; 1155.7 \mathrm{~cm}^{-1} ;{ }^{1} \mathrm{H}$ NMR $(500$ $\left.\mathrm{MHz} \mathrm{CDCl}_{3}\right) \delta 11.84(\mathrm{bs}, 1 \mathrm{H}) ; 7.78(\mathrm{~s}, 1 \mathrm{H}) ; 7.52-7.48(\mathrm{~m}, 2 \mathrm{H}) ; 7.43-7.21$ (AA'BB', $4 \mathrm{H}) ; 7.04(\mathrm{~d}, J=7.0 \mathrm{~Hz}, 1 \mathrm{H}) ; 6.83(\mathrm{t}, J=7.3 \mathrm{~Hz}, 1 \mathrm{H}) ; 1.41(\mathrm{~s}, 9 \mathrm{H}) ;{ }^{13} \mathrm{C}$ NMR $(125 \mathrm{MHz}$, $\left.\mathrm{CDCl}_{3}\right) \delta 201.3,163.5,162.9,140.8,137.4,132.5,132.3,131.9,131.8,131.6,125.3$, 120.0, 119.7, 118.7, 83.1, 28.1; LRMS (electrospray): Mass calculated for $\mathrm{C}_{20} \mathrm{H}_{19} \mathrm{BrO}_{4}$, $[\mathrm{M}]^{+}, 402.1,404.0$. Found $[\mathrm{M}+23]^{+}, 424.7$, 426.8. An NOE difference experiment was conducted for this compound and a positive NOE was seen for the alkene proton when the tert-butyl signal was irradiated and a positive NOE was seen for the tert-butyl signal upon irradiation of the alkene proton. This compound was further characterized by X-ray crystal structure, see $S 56$.

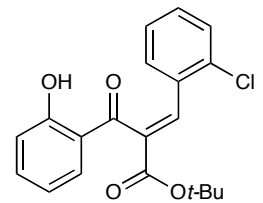

(E)-tert-butyl 3-(2-chlorophenyl)-2-(2-hydroxyphenylcarbonyl)prop2-enoate: Prepared according to general procedure A using tert-butyl 3(2-hydroxyphenyl)-3-oxopropanoate (18) (236 mg, $1.0 \mathrm{mmol}), 2-$ chlorobenzaldehyde $(113 \mu \mathrm{L}, 1.0 \mathrm{mmol}) 15 \mathrm{~mL}$ benzene, piperidine $(10$ $\mu \mathrm{L}, 0.1 \mathrm{mmol})$ and glacial acetic acid $(5.6 \mu \mathrm{L}, 0.1 \mathrm{mmol})$, refluxed for 90 min. Purified via recrystallization from hexanes $/ \mathrm{CH}_{2} \mathrm{Cl}_{2}$, yielding $72 \mathrm{mg}(20 \%)$ of a white solid. $\mathrm{mp}=75-78{ }^{\circ} \mathrm{C}$ IR (film) $2979.0 ; 1720.5 ; 1630.9 ; 1156.3 \mathrm{~cm}^{-1 ; 1} \mathrm{H}$ NMR (500 $\left.\mathrm{MHz}, \mathrm{CDCl}_{3}\right) \delta 11.80$ (bs, $\left.1 \mathrm{H}\right) ; 8.19(\mathrm{~s}, 1 \mathrm{H}) ; 7.50$ (d, J=7.9 Hz, 1H); 7.44 (t, J=7.9 Hz, $1 \mathrm{H}) ; 7.36(\mathrm{~d}, J=7.9 \mathrm{~Hz}, 1 \mathrm{H}) ; 7.26(\mathrm{~d}, J=7.9 \mathrm{~Hz}, 1 \mathrm{H}) ; 7.21(\mathrm{t}, J=7.6 \mathrm{~Hz}, 1 \mathrm{H}) ; 7.08(\mathrm{t}, J=$ 7.06, Hz, 1H); $6.97(\mathrm{~d}, J=7.9 \mathrm{~Hz}, 1 \mathrm{H}) ; 6.82(\mathrm{t}, J=7.6 \mathrm{~Hz}, 1 \mathrm{H}) ; 1.43(\mathrm{~s}, 9 \mathrm{H}) ;{ }^{13} \mathrm{C} \mathrm{NMR}$ $\left(125 \mathrm{MHz}, \mathrm{CDCl}_{3}\right) \delta 200.6,163.3,162.8,139.1,137.2,135.0,134.0,131.9$ (double intensity), 131.3, 130.2, 130.1, 127.2, 120.0, 119.5, 118.5, 83.1, 28.1; LRMS (electrospray): Mass calculated for $\mathrm{C}_{20} \mathrm{H}_{19} \mathrm{ClO}_{4}[\mathrm{M}]^{+}, 358.09$. Found $[\mathrm{M}+23]^{+}, 381.5$.

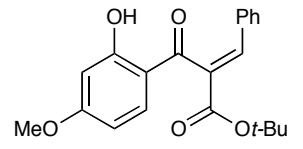

(E)-tert-butyl

2-(2-hydroxy-4-methoxyphenylcarbonyl)-3phenylprop-2-enoate: Prepared according to general procedure A using tert-butyl 3-(2-hydroxy4-methoxyphenyl)-3-oxopropanoate (532 $\mathrm{mg}, 2.0 \mathrm{mmol})$, benzaldehyde $(200 \mu \mathrm{L}, 2.00 \mathrm{mmol}), 12.0 \mathrm{~mL}$ benzene, piperidine $(10 \mu \mathrm{L}$, $0.10 \mathrm{mmol})$ and glacial acetic acid $(5.7 \mu \mathrm{L}, 0.10 \mathrm{mmol})$, refluxed for $3 \mathrm{~h}$. Purified via recrystallization from hexanes $/ \mathrm{CH}_{2} \mathrm{Cl}_{2}$, yielding $321 \mathrm{mg}(46 \%)$ of white solid. $\mathrm{mp}=105$ $107{ }^{\circ} \mathrm{C}$; IR (film) 2977.5; 1712.8; 1624.0; $1155.6 \mathrm{~cm}^{-1} ;{ }^{1} \mathrm{H} \mathrm{NMR}\left(500 \mathrm{MHz}, \mathrm{CDCl}_{3}\right) \delta$ 12.41 (bs, 1H), $7.81(\mathrm{~s}, 1 \mathrm{H}), 7.41(\mathrm{~d}, J=9.1 \mathrm{~Hz}, 1 \mathrm{H}), 7.37-7.25(\mathrm{~m}, 5 \mathrm{H}), 6.46$ (d, $J=2.1$ $\mathrm{Hz}, 1 \mathrm{H}), 6.34(\mathrm{dd}, J=8.8,2.1 \mathrm{~Hz}, 1 \mathrm{H}), 3.83(\mathrm{~s}, 3 \mathrm{H}), 1.42(\mathrm{~s}, 9 \mathrm{H}) ;{ }^{13} \mathrm{C}$ NMR $(125 \mathrm{MHz}$, $\left.\mathrm{CDCl}_{3}\right) \delta 199.4,166.8,165.8,164.0,141.8,133.6,133.1,131.6,130.5,130.3,129.1$, 114.3, 108.4, 101.1, 82.6, 55.8, 28.1; LRMS (electrospray): Mass calculated for $\mathrm{C}_{21} \mathrm{H}_{22} \mathrm{O}_{5}$ $[\mathrm{M}]^{+}, 354.14$. Found $[\mathrm{M}+23]^{+}, 377.5$. 


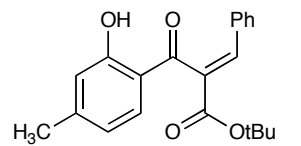

(E)-tert-butyl

2-(2-hydroxy-4-methylphenylcarbonyl)-3phenylprop-2-enoate: Prepared according to general procedure A using tert-butyl 3-(2-hydroxy4-methyphenyl)-3-oxopropanoate (250 $\mathrm{mg}, 1.0 \mathrm{mmol})$, benzaldehyde $(100 \mu \mathrm{L}, 1.00 \mathrm{mmol}), 12.0 \mathrm{~mL}$ benzene, piperidine $(10 \mu \mathrm{L}$, $0.10 \mathrm{mmol})$ and glacial acetic acid $(5.7 \mu \mathrm{L}, 0.10 \mathrm{mmol})$, refluxed for $3 \mathrm{~h}$. Purified via recrystallization from hexanes/ $\mathrm{CH}_{2} \mathrm{Cl}_{2}$, yielding $154 \mathrm{mg}(46 \%)$ of white solid. $\mathrm{mp}=92$ $95{ }^{\circ} \mathrm{C}$; IR (film) 2977.9; 1718.2; 1627.9; $1155.0 \mathrm{~cm}^{-1} ;{ }^{1} \mathrm{H}$ NMR $\left(500 \mathrm{MHz}, \mathrm{CDCl}_{3}\right) \delta$ 11.95 (bs, $1 \mathrm{H}), 7.82(\mathrm{~s}, 1 \mathrm{H}), 7.40(\mathrm{~d}, J=9.6 \mathrm{~Hz}, 1 \mathrm{H}), 7.36-7.29(\mathrm{~m}, 5 \mathrm{H}), 6.83(\mathrm{~s}, 1 \mathrm{H})$, $6.61(\mathrm{~d}, J=8.0 \mathrm{~Hz}, 1 \mathrm{H}), 2.34(\mathrm{~s}, 3 \mathrm{H}), 1.41(\mathrm{~s}, 9 \mathrm{H}) ;{ }^{13} \mathrm{C} \mathrm{NMR}\left(125 \mathrm{MHz}, \mathrm{CDCl}_{3}\right) \delta 200.8$, 163.9, 163.0, 149.0, 142.0, 133.0, 131.8, 131.7, 130.6, 130.3, 129.1, 120.9, 118.5, 118.0, 82.7, 28.1, 22.3; LRMS (electrospray): Mass calculated for $\mathrm{C}_{21} \mathrm{H}_{22} \mathrm{O}_{4}[\mathrm{M}]^{+}, 338.15$. Found $[\mathrm{M}+23]^{+}, 361.2$. An NOE difference experiment was conducted for this compound and a positive NOE was seen for the alkene proton when the tert-butyl signal was irradiated and a positive NOE was seen for the tert-butyl signal upon irradiation of the alkene proton.

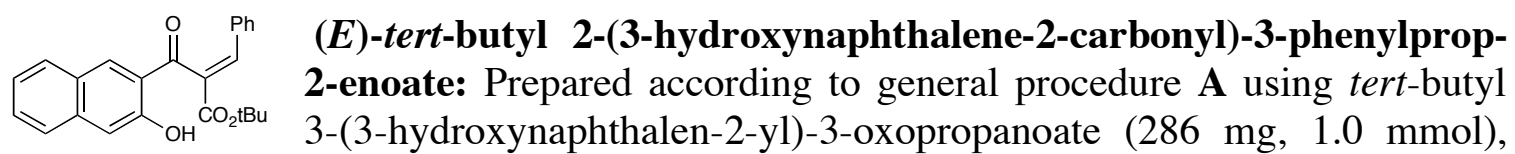
benzaldehyde (100 $\mu \mathrm{L}, 1.00 \mathrm{mmol}), 15.0 \mathrm{~mL}$ benzene, piperidine $(10 \mu \mathrm{L}, 0.10 \mathrm{mmol})$ and glacial acetic acid $(5.7 \mu \mathrm{L}, 0.10 \mathrm{mmol})$, refluxed for $4 \mathrm{~h}$. Purified via recrystallization from hexanes $/ \mathrm{CH}_{2} \mathrm{Cl}_{2}$, yielding $213 \mathrm{mg}(57 \%)$ of yellow solid. $\mathrm{mp}=132-135{ }^{\circ} \mathrm{C}$; IR (film) 2977.6; 1716.6; 1639.5; $1154.5 \mathrm{~cm}^{-1} ;{ }^{1} \mathrm{H}$ NMR $\left(500 \mathrm{MHz}, \mathrm{CDCl}_{3}\right) \delta 11.20$ (bs, $1 \mathrm{H}), 8.19(\mathrm{~s}, 1 \mathrm{H}) ; 7.96(\mathrm{~s}, 1 \mathrm{H}), 7.70(\mathrm{t}, J=7.3 \mathrm{~Hz}, 2 \mathrm{H}), 7.51(\mathrm{t}, J=8.0 \mathrm{~Hz}, 1 \mathrm{H}), 7.40-7.25$ (m, 7H), $1.38(\mathrm{~s}, 9 \mathrm{H}) ;{ }^{13} \mathrm{C}$ NMR $\left(125 \mathrm{MHz}, \mathrm{CDCl}_{3}\right) \delta$ 201.9, 163.9, 157.1, 142.8, 138.7, 135.1, 132.8, 131.6, 130.8, 130.4, 130.2, 129.8, 129.2, 127.3, 126.6, 124.4, 121.7, 112.6, 83.0, 28.1; LRMS (electrospray): Mass calculated for $\mathrm{C}_{24} \mathrm{H}_{22} \mathrm{O}_{4}[\mathrm{M}]^{+}$, 374.15. Found $[\mathrm{M}+23]^{+}, 397.0$.

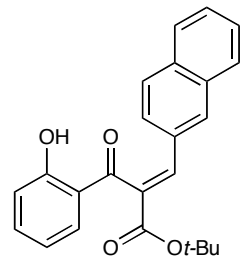

(E)-tert-butyl 2-(2-hydroxyphenylcarbonyl)-3-(naphthalen-2-yl)prop2-enoate: Prepared according to general procedure B using tert-butyl 3(2-hydroxyphenyl)-3-oxopropanoate (18) (259 mg, $1.1 \mathrm{mmol}), 2$ naphthaldehyde (172 mg, $1.1 \mathrm{mmol}), 12.0 \mathrm{~mL}$ toluene, piperidinium acetate $(2.2 \mathrm{~mL}, 0.025 \mathrm{M}$ in toluene) and sodium sulfate $(2.3 \mathrm{~g})$, stirred at $22{ }^{\circ} \mathrm{C}$ for $7 \mathrm{~d}$. Purified via recrystallization from hexanes $/ \mathrm{CH}_{2} \mathrm{Cl}_{2}$, yielding $240 \mathrm{mg}(58 \%)$ of beige crystals. $\mathrm{mp}=111-112{ }^{\circ} \mathrm{C}$; IR (film) 2978.0; 1712.8; 1624.1; $1154.1 \mathrm{~cm}^{-1} ;{ }^{1} \mathrm{H}$ NMR $\left(500 \mathrm{MHz}, \mathrm{CDCl}_{3}\right) \delta 11.97$ (bs, $\left.1 \mathrm{H}\right) ; 8.02$ (s, $\left.1 \mathrm{H}\right) ; 7.91$ (s, $1 \mathrm{H}) ; 7.79(\mathrm{~d}, J=7.6 \mathrm{~Hz}, 1 \mathrm{H}) ; 7.78(\mathrm{~d}, J=7.3 \mathrm{~Hz}, 1 \mathrm{H}) ; 7.70(\mathrm{~d}, J=8.5 \mathrm{~Hz}, 1 \mathrm{H}) ; 7.58$ (dd, $J=8.0,2.5 \mathrm{~Hz}, 1 \mathrm{H}) ; 7.51-7.46(\mathrm{~m}, 3 \mathrm{H}) ; 7.37(\mathrm{dd}, J=8.5,1.5 \mathrm{~Hz}, 1 \mathrm{H}) ; 7.05(\mathrm{~d}, J=8.2$ $\mathrm{Hz}, 1 \mathrm{H}) ; 6.81$ (t, $J=7.3 \mathrm{~Hz}, 1 \mathrm{H}) ; 1.44(\mathrm{~s}, 9 \mathrm{H}) ;{ }^{13} \mathrm{C} \mathrm{NMR}\left(125 \mathrm{MHz}, \mathrm{CDCl}_{3}\right) \delta 201.9$, $163.9,162.9,142.4,137.3,134.1,133.2,132.05,132.03,131.6,130.6,129.0$ (x2), 128.0, $127.9,127.0,125.9,120.3,119.6,118.6,82.8,28.2$; LRMS (electrospray): Mass calculated for $\mathrm{C}_{24} \mathrm{H}_{22} \mathrm{O}_{4}[\mathrm{M}]^{+}, 374.14$. Found $[\mathrm{M}+23]^{+}$, 397.2. 


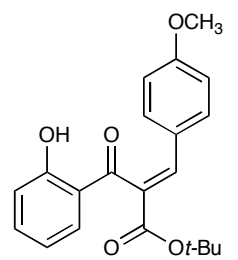

(E)-tert-butyl 2-(2-hydroxyphenylcarbonyl)-3-(4-methoxyphenyl)prop2-enoate: Prepared according to general procedure B using tert-butyl 3(2-hydroxyphenyl)-3-oxopropanoate (18) (236 $\mathrm{mg}, 1.0 \mathrm{mmol}), \quad p$ anisaldehyde $(115 \mu \mathrm{L}, 1.0 \mathrm{mmol}), 12.0 \mathrm{~mL}$ toluene, piperidinium acetate $\left(0.3 \mathrm{~mL}, 0.025 \mathrm{M}\right.$ in toluene) and sodium sulfate $(2.0 \mathrm{~g})$, stirred at $22{ }^{\circ} \mathrm{C}$ for $7 \mathrm{~d}$. Purified via recrystallization from hexanes $/ \mathrm{CH}_{2} \mathrm{Cl}_{2}$, yielding 250 $\operatorname{mg}(71 \%)$ of white crystals. $\mathrm{mp}=129-132{ }^{\circ} \mathrm{C}$; IR (film) $2982.5 ; 1708.9 ; 1603.8 ; 1246.8$; $1155.0 \mathrm{~cm}^{-1} ;{ }^{1} \mathrm{H}$ NMR $\left(500 \mathrm{MHz}, \mathrm{CDCl}_{3}\right) \delta 11.97$ (bs, $\left.1 \mathrm{H}\right) ; 7.80(\mathrm{~s}, 1 \mathrm{H}) ; 7.56(\mathrm{~d}, J=8.0$ $\mathrm{Hz}, 1 \mathrm{H}) ; 7.48$ (t, $J=7.4 \mathrm{~Hz}, 1 \mathrm{H}) ; 7.32-6.79$ (AA'BB', 4H), 7.04 (t, $J=8.6 \mathrm{~Hz}, 1 \mathrm{H}), 6.82$ $(\mathrm{m}, 1 \mathrm{H}), 3.78(\mathrm{~s}, 3 \mathrm{H}) ; 1.40(\mathrm{~s}, 9 \mathrm{H}) ;{ }^{13} \mathrm{C} \mathrm{NMR}\left(125 \mathrm{MHz}, \mathrm{CDCl}_{3}\right) \delta$ 202.3, 164.1, 162.9, 161.6, 142.0, 137.1, 132.4, 132.0, 128.9, 125.3, 120.2, 119.5, 118.5, 114.7, 82.5, 55.6, 28.1; LRMS (electrospray): Mass calculated for $\mathrm{C}_{21} \mathrm{H}_{22} \mathrm{O}_{5}[\mathrm{M}]^{+}, 354.14$. Found $[\mathrm{M}+23]^{+}$, 377.5. An NOE difference experiment was conducted for this compound and a positive NOE was seen for the alkene proton when the tert-butyl signal was irradiated and a positive NOE was seen for the tert-butyl signal upon irradiation of the alkene proton.

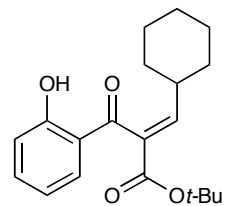

\section{(E)-tert-butyl}

3-cyclohexyl-2-(2-hydroxyphenylcarbonyl)prop-2enoate: Prepared according to general procedure B using tert-butyl 3-(2hydroxyphenyl)-3-oxopropanoate (18) (236 $\mathrm{mg}, \quad 1.0 \mathrm{mmol})$, cyclohexanecarboxaldehyde $(120 \mu \mathrm{L}, 1.0 \mathrm{mmol}) \quad 7.0 \mathrm{~mL}$ benzene, piperidinium acetate $(2.0 \mathrm{~mL}, 0.025 \mathrm{M}$ in toluene) and sodium sulfate $(2.1$ g), stirred at $22{ }^{\circ} \mathrm{C}$ for $8 \mathrm{~d}$. Purified via column chromatography with $5 \%$ EtOAc/hexanes, yielding $165 \mathrm{mg}(50 \%)$ of a white solid. $\mathrm{R}_{f}=0.59$ (10\% ether/hexanes); $\mathrm{mp}=62-65{ }^{\circ} \mathrm{C}$; IR (film) 2929.2; 1716.6; 1631.9; 1257.5; $1153.6 \mathrm{~cm}^{-1} ;{ }^{1} \mathrm{H}$ NMR (500 $\left.\mathrm{MHz}, \mathrm{CDCl}_{3}\right) \delta$ $11.92(\mathrm{~s}, 1 \mathrm{H}) ; 7.51-7.46(\mathrm{~m}, 2 \mathrm{H}) ; 7.02(\mathrm{~d}, \mathrm{~J}=8.2 \mathrm{~Hz}, 1 \mathrm{H}) ; 6.90-6.86(\mathrm{~m}, 2 \mathrm{H}) ; 2.11(\mathrm{~m}$, $1 \mathrm{H}) ; 1.68-1.60(\mathrm{~m}, 5 \mathrm{H}) ; 1.35(\mathrm{~s}, 9 \mathrm{H}) ; 1.20-1.15(\mathrm{~m}, 5 \mathrm{H}) .{ }^{13} \mathrm{C} \mathrm{NMR}\left(125 \mathrm{MHz}, \mathrm{CDCl}_{3}\right) \delta$ 200.8, 163.7, 162.6, 152.2, 136.9, 132.3, 124.6, 120.5, 119.2, 118.4, 82.3, 31.9, 28.5, 28.0, 25.8, 25.1; LRMS (electrospray): Mass calculated for $\mathrm{C}_{20} \mathrm{H}_{26} \mathrm{O}_{4}[\mathrm{M}]^{+}, 330.18$. Found $[\mathrm{M}+23]^{+}, 353.6$.

\section{General Cyclization Procedure:}

To a $10 \mathrm{~mL}$ round bottom flask containing a magnetic stirring bar was added alkylidene compound, 10 mol\% thiourea catalyst III. The flask is purged with $\mathrm{N}_{2}$. The solids were dissolved in toluene and the resulting solution was stored at $-25^{\circ} \mathrm{C}$. Reaction progress was monitored by RP HPLC. After completion of reaction, the solution was diluted with EtOAc $(20 \mathrm{~mL})$, poured into a separatory funnel and washed with brine. The organic layer was dried over anhydrous $\mathrm{Na}_{2} \mathrm{SO}_{4}$, filtered and concentrated to provide the crude flavanone. The crude mixture was run through a silica gel column (10\% EtOAc/hexanes).

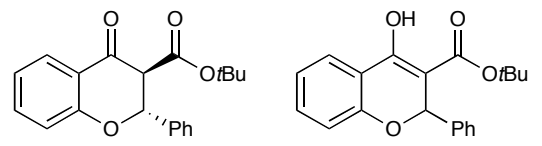

$(2 R, 3 S)-t$-butyl

4-oxo-2-phenylchroman-3carboxylate and $t$-butyl 4-hydroxy-2-phenyl-2Hchromene-3-carboxylate 6: Prepared according to general procedure using (E)-tert-butyl 2-(2-hydroxyphenylcarbonyl)-3-phenylprop-2enoate 5 (65 mg, $0.2 \mathrm{mmol})$ and thiourea catalyst III (14 mg, $0.02 \mathrm{mmol})$ in $2 \mathrm{~mL}$ toluene for 36 hours to afford $55 \mathrm{mg}(85 \%)$ of $\mathbf{6}$ as a pink solid in $92 \%$ ee. Analytical data for $\mathbf{6}$, ${ }^{1} \mathrm{H}$ NMR $\left(500 \mathrm{MHz}, \mathrm{CDCl}_{3}\right) \delta$ for trans: $7.95(\mathrm{~d}, J=7.3 \mathrm{~Hz}, 1 \mathrm{H}), 7.53-7.24(\mathrm{~m}, 6 \mathrm{H}), 7.08$ 
(t, $J=7.3 \mathrm{~Hz}, 1 \mathrm{H}), 7.03(\mathrm{~d}, J=8.3 \mathrm{~Hz}, 1 \mathrm{H}), 5.62(\mathrm{~d}, J=12.2 \mathrm{~Hz}, 1 \mathrm{H}), 3.98(\mathrm{~d} J=12.2$ $\mathrm{Hz}, 1 \mathrm{H}), 1.30(\mathrm{~s}, 9 \mathrm{H})$; some enol tautomer may be present, depending on concentration: 12.49 (bs, $1 \mathrm{H}), 7.66(\mathrm{~d}, J=7.3,1 \mathrm{H}), 7.53-7.24(\mathrm{~m}, 6 \mathrm{H}) 6.94(\mathrm{t}, J=7.6 \mathrm{~Hz}, 1 \mathrm{H}), 6.79(\mathrm{~d}$, $J=8.2 \mathrm{~Hz}, 1 \mathrm{H}), 6.18(\mathrm{~s}, 1 \mathrm{H}), 1.42(\mathrm{~s}, 9 \mathrm{H}) .{ }^{13} \mathrm{C} \mathrm{NMR}\left(125 \mathrm{MHz}, \mathrm{CDCl}_{3}\right) \delta 188.5,166.2$, $161.3,136.78,136.76,129.7,128.9,127.9,127.7,122.2,120.3,118.3,82.6,81.8,60.6$, 28.0 .

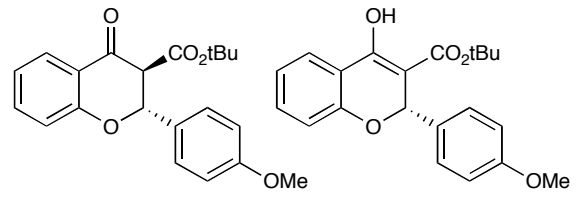

$(2 R, 3 S)-t$-butyl

4-0xo-2-(4methoxyphenyl)chroman-3-carboxylate and $t$ butyl 4-hydroxy-2-4-methoxyphenyl)-2Hchromene-3-carboxylate: Prepared according to general procedure using (E)-tert-butyl 2-(2-hydroxyphenylcarbonyl)-3-phenylprop-2enoate $(27 \mathrm{mg}, 0.08 \mathrm{mmol})$ and thiourea catalyst III $(5 \mathrm{mg}, 0.008 \mathrm{mmol})$ in $0.8 \mathrm{~mL}$ toluene for 24 hours to afford $25 \mathrm{mg}(94 \%)$ of a white solid in $91 \%$ ee. Analytical data for 12, ${ }^{1} \mathrm{H}$ NMR $\left(500 \mathrm{MHz}, \mathrm{CDCl}_{3}\right) \delta$ for trans: $7.95(\mathrm{~d}, J=7.6 \mathrm{~Hz}, 1 \mathrm{H}), 7.50(\mathrm{t}, J=7.9$ $\mathrm{Hz}, 1 \mathrm{H}), 7.46-6.95$ (AA'BB', 4H), 7.06 (t, $J=7.3 \mathrm{~Hz}, 1 \mathrm{H}), 7.01(\mathrm{~d}, J=8.2 \mathrm{~Hz}, 1 \mathrm{H}), 5.57$ (d, $J=12.5 \mathrm{~Hz}, 1 \mathrm{H}), 3.98(\mathrm{~d} J=12.5 \mathrm{~Hz}, 1 \mathrm{H}), 3.82(\mathrm{~s}, 3 \mathrm{H}), 1.32(\mathrm{~s}, 9 \mathrm{H})$; enol tautomer: 12.53 (bs, $1 \mathrm{H}), 7.67$ (d, $J=7.6,1 \mathrm{H}), 7.30-6.79\left(\mathrm{AA}^{\prime} \mathrm{BB}^{\prime}, 4 \mathrm{H}\right) 7.23$ (t, $\left.J=8.0 \mathrm{~Hz}, 1 \mathrm{H}\right)$, $6.92(\mathrm{~m}, 1 \mathrm{H}), 6.13(\mathrm{~s}, 1 \mathrm{H}), 3.74,(\mathrm{~s}, 3 \mathrm{H}), 1.42(\mathrm{~s}, 9 \mathrm{H})$.

\section{General Cyclization/Decarboxylation Procedure:}

To a $10 \mathrm{~mL}$ round bottom flask containing a magnetic stirring bar was added alkylidene compound, $10 \mathrm{~mol} \%$ thiourea catalyst III. The flask is purged with $\mathrm{N}_{2}$. The solids were dissolved in toluene and the resulting solution was stored at $-25^{\circ} \mathrm{C}$. Reaction progress was monitored by RP HPLC. After complete cyclization, $50 \mathrm{~mol} \%$-toluenesulfonic acid was added to the flask and the solution was heated to $80^{\circ} \mathrm{C}$, and the reaction was monitored by TLC. Upon completion of the reaction, the solution was allowed to cool and the solution was diluted with EtOAc $(20 \mathrm{~mL})$, poured into a separatory funnel and washed with brine. The organic layer was dried over anhydrous $\mathrm{Na}_{2} \mathrm{SO}_{4}$, filtered and concentrated to provide the crude flavanone. The crude mixture was purified via silica gel chromatography (10\% EtOAc/hexanes) and concentrated in vacuo.

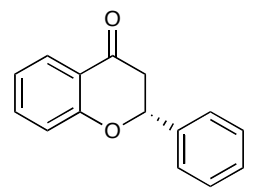

(R)-flavanone (7): Prepared according to general procedure using $(E)$ tert-butyl 2-(2-hydroxyphenylcarbonyl)-3-phenylprop-2-enoate (5) (65 $\mathrm{mg}, 0.2 \mathrm{mmol})$ and thiourea catalyst III $(14 \mathrm{mg}, 0.02 \mathrm{mmol})$ in $2.0 \mathrm{~mL}$ toluene for $36 \mathrm{~h}$ at $-25{ }^{\circ} \mathrm{C}$ and $p$-toluenesulfonic acid $(19 \mathrm{mg}, 0.10 \mathrm{mmol})$ for $24 \mathrm{~h}$. Purification via column chromatography with $10 \% \mathrm{EtOAc} /$ hexanes afforded 41 $\mathrm{mg}(92 \%)$ of 7 as a white solid in $94 \%$ ee. $[\alpha]_{\mathrm{D}}:+55.6(\mathrm{EtOH}, \mathrm{c}=0.5)$. Analytical data match those reported in the literature. ${ }^{6}$

\footnotetext{
${ }^{6}$ Ramadas, S.; Krupadanam, G. L. D. Tetrahedron: Asymmetry 2004, 15, 3381-3391.
} 


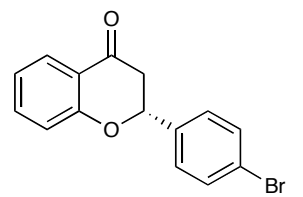

2-(4-bromophenyl)chroman-4-one (8): Prepared according to general procedure using E-tert-butyl 3-(4-bromophenyl)-2-(2hydroxyphenylcarbonyl)prop-2-enoate $(81.0 \mathrm{mg}, 0.20 \mathrm{mmol})$, thiourea catalyst III (14 mg, $0.020 \mathrm{mmol})$ in $2.0 \mathrm{~mL}$ toluene for $38 \mathrm{~h}$ at $-25^{\circ} \mathrm{C}$ and $p$-toluenesulfonic acid $(19 \mathrm{mg}, 0.10 \mathrm{mmol})$ for $36 \mathrm{~h}$. Purification via column chromatography with $10 \%$ EtOAc/hexanes afforded $39.5 \mathrm{mg}(65 \%)$ of $\mathbf{8}$ as a white solid in $92 \%$ ee. $\mathrm{mp}=115-116{ }^{\circ} \mathrm{C} .[\alpha]_{\mathrm{D}}:+50.4(\mathrm{EtOH}, \mathrm{c}=0.5)$. Analytical data for 8: IR (film) 1691.8, 1604.8, 1465.1, 1304.6, $1222.9 \mathrm{~cm}^{-1} ;{ }^{1} \mathrm{H}$ NMR $\left(500 \mathrm{MHz}, \mathrm{CDCl}_{3}\right) \delta 7.93$ $(\mathrm{d}, J=7.5 \mathrm{~Hz}, 1 \mathrm{H}), 7.58-7.36\left(\mathrm{AA}^{\prime} \mathrm{BB}^{\prime}, 4 \mathrm{H}\right), 7.52(\mathrm{t}, J=8.0 \mathrm{~Hz}, 1 \mathrm{H}), 7.09-7.05(\mathrm{~m}, 2 \mathrm{H})$, $5.45(\mathrm{dd}, J=13.2,2.4 \mathrm{~Hz}, 1 \mathrm{H}), 3.00(\mathrm{dd}, J=16.8,13.3 \mathrm{~Hz}, 1 \mathrm{H}), 2.88(\mathrm{dd}, J=16.8,2.6$ $\mathrm{Hz}, 1 \mathrm{H}) ;{ }^{13} \mathrm{C}$ NMR $\left(125 \mathrm{MHz}, \mathrm{CDCl}_{3}\right) \delta 191.7,161.5,138.0,136.6,132.3,128.1,127.3$, 123.0, 122.1, 121.1, 118.3, 79.1, 44.8; LRMS (electrospray): Exact mass calcd for $\mathrm{C}_{15} \mathrm{H}_{11} \mathrm{BrO}_{2}[\mathrm{M}]^{+}, 301.99$, 303.99. Found [M+1], 305.5.

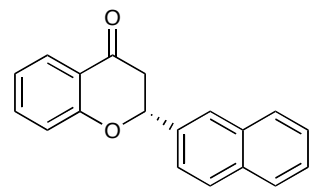

(R)-2-(2-naphthyl)chroman-4-one (9): Prepared according to general procedure using $(E)$-tert-butyl 2-(2-hydroxyphenylcarbonyl)3-(naphthalen-2-yl)prop-2-enoate (77 $\mathrm{mg}, 0.20 \mathrm{mmol}$ ), thiourea catalyst III (14 mg, $0.020 \mathrm{mmol})$ in $2.0 \mathrm{~mL}$ toluene for $38 \mathrm{~h}$ at -25 ${ }^{\circ} \mathrm{C}$ and $p$-toluenesulfonic acid $(19 \mathrm{mg}, 0.10 \mathrm{mmol})$ for $10 \mathrm{~h}$. Purification via column chromatography with $10 \%$ EtOAc/hexanes afforded $49 \mathrm{mg}(89 \%)$ of $\mathbf{9}$ as a white solid in $91 \%$ ee. $[\alpha]_{\mathrm{D}}:+56.3(\mathrm{EtOH}, \mathrm{c}=0.5)$. Analytical data for 9: IR (film) 1690.9, 1607.9, 1464.8, 1304.4, $1226.9 \mathrm{~cm}^{-1} ;{ }^{1} \mathrm{H}$ NMR (500 MHz, $\mathrm{CDCl}_{3}$ ) $\delta$ 7.99-7.89 (m, 5H), 7.63-7.54 $(\mathrm{m}, 4 \mathrm{H}), 7.13-7.09(\mathrm{~m}, 2 \mathrm{H}), 5.68(\mathrm{dd}, J=13.1,2.4 \mathrm{~Hz}, 1 \mathrm{H}), 3.22(\mathrm{dd}, J=16.8,13.4 \mathrm{~Hz}$, $1 \mathrm{H}), 3.00(\mathrm{dd}, J=16.8,2.7 \mathrm{~Hz}, 1 \mathrm{H}) ;{ }^{13} \mathrm{C} \mathrm{NMR}\left(125 \mathrm{MHz}, \mathrm{CDCl}_{3}\right) \delta 192.2,161.8,136.5$, 136.3, 133.6, 133.4, 129.0, 128.4, 128.0, 127.3, 126.8 (x2), 125.6, 123.9, 121.9, 121.2, 118.4, 79.9, 44.9; LRMS (electrospray): Exact mass calcd. for $\mathrm{C}_{19} \mathrm{H}_{14} \mathrm{O}_{2}[\mathrm{M}]^{+}, 274.10$. Found $[\mathrm{M}]^{+}, 273.8$.

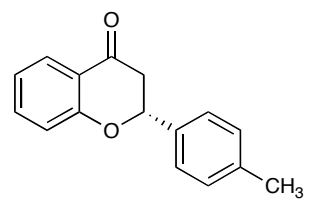

(R)- 2-p-tolylchroman-4-one (10): Prepared according to general procedure using (E)-tert-butyl 2-(2-hydroxyphenylcarbonyl)-3-ptolylprop-2-enoate $(54 \mathrm{mg}, 0.16 \mathrm{mmol})$, thiourea catalyst III $(11 \mathrm{mg}$, $0.016 \mathrm{mmol})$ in $1.6 \mathrm{~mL}$ toluene for $36 \mathrm{~h}$ at $-25{ }^{\circ} \mathrm{C}$ and $p$ toluenesulfonic acid (15 mg, $0.08 \mathrm{mmol}$ ) for $25 \mathrm{~h}$. Purification via column chromatography with $10 \%$ EtOAc/hexanes afforded $32 \mathrm{mg}(83 \%)$ of $\mathbf{1 0}$ as a white solid in $90 \%$ ee. Analytical data match those reported in the literature. ${ }^{6}$

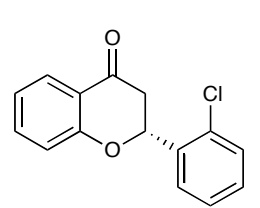

(R)-2-(2-chlorophenyl)chroman-4-one (11): Prepared according to general procedure using (E)-tert-butyl 3-(2-chlorophenyl)-2-(2hydroxyphenylcarbonyl)prop-2-enoate $(36 \mathrm{mg}, 0.10 \mathrm{mmol})$, thiourea catalyst III $(7 \mathrm{mg}, 0.010 \mathrm{mmol})$ in $1.0 \mathrm{~mL}$ toluene for $36 \mathrm{~h}$ at $-25^{\circ} \mathrm{C}$ and p-toluenesulfonic acid $(10 \mathrm{mg}, 0.05 \mathrm{mmol})$ for $50 \mathrm{~h}$. Purification via column chromatography with 10\% EtOAc/hexanes afforded $19 \mathrm{mg}(67 \%)$ of 11 as a white solid in $88 \%$ ee. Analytical data match those reported in the literature. ${ }^{6}$ 


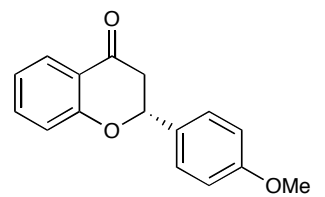

(R)-2-(4-methoxyphenyl)chroman-4-one: Prepared according to general procedure using $(E)$-tert-butyl 2-(2-hydroxyphenylcarbonyl)3-(4-methoxyphenyl)prop-2-enoate $(35.0 \mathrm{mg}, 0.10 \mathrm{mmol})$, thiourea catalyst III ( $7 \mathrm{mg}, 0.01 \mathrm{mmol})$ in $1.0 \mathrm{~mL}$ toluene for $3 \mathrm{~d}$ at $-25{ }^{\circ} \mathrm{C}$. After completion of reaction, $100 \mathrm{~mL}$ sample was withdrawn to determine ee of 3-tertbutylcarboxy-(4-methoxyphenyl)chromanone 12 (90\% ee). Anhydrous $\mathrm{MgBr}_{2} \bullet \mathrm{OEt}_{2}$ (170 $\mathrm{mg}$ ) was added to the remaining solution and stirred at RT for $48 \mathrm{~h}$. Purification via column chromatography with $10 \%$ EtOAc/hexanes afforded $11 \mathrm{mg}(49 \%)$ as a yellow solid in $78 \%$ ee. Analytical data match those reported in the literature. ${ }^{6}$

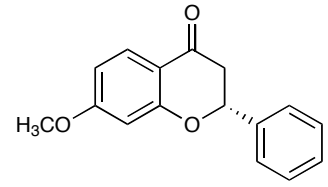

(R)-7-methoxy-2-phenylchroman-4-one (13): Prepared according to general procedure using (E)-tert-butyl 2-(2-hydroxy-4methoxyphenylcarbonyl)-3-phenylprop-2-enoate $(71 \mathrm{mg}, \quad 0.20$ $\mathrm{mmol})$, thiourea catalyst III (14 $\mathrm{mg}, 0.020 \mathrm{mmol})$ in $2.0 \mathrm{~mL}$ toluene for $42 \mathrm{~h}$ at $-25^{\circ} \mathrm{C}$ and $p$-toluenesulfonic acid $(19 \mathrm{mg}, 0.10 \mathrm{mmol})$ for $18 \mathrm{~h}$. Purification via column chromatography with $10 \%$ EtOAc/hexanes afforded $36 \mathrm{mg}(71 \%)$ of 13 as a white solid in $89 \%$ ee. $[\alpha]_{\mathrm{D}}:+58.5(\mathrm{EtOH}, \mathrm{c}=0.5)$. Analytical data for 13: IR (film) 1683.3, 1608.6, 1442.7, $1258.9 \mathrm{~cm}^{-1} ;{ }^{1} \mathrm{H}$ NMR $\left(500 \mathrm{MHz}, \mathrm{CDCl}_{3}\right) \delta 7.89(\mathrm{~d}, J=8.5 \mathrm{~Hz}$, $1 \mathrm{H}), 7.50-7.39(\mathrm{~m}, 5 \mathrm{H}), 6.63(\mathrm{dd}, J=8.8,2.1 \mathrm{~Hz}, 1 \mathrm{H}), 6.52(\mathrm{~d}, J=1.5 \mathrm{~Hz}, 1 \mathrm{H}), 5.48$ (dd, $J=13.4,2.4 \mathrm{~Hz}, 1 \mathrm{H}), 3.85$ (s, 3H), $3.06(\mathrm{dd}, J=16.8,13.4 \mathrm{~Hz}, 1 \mathrm{H}), 2.85(\mathrm{dd}, J=16.8$, $2.8 \mathrm{~Hz}, 1 \mathrm{H}) ;{ }^{13} \mathrm{C} \mathrm{NMR}\left(125 \mathrm{MHz}, \mathrm{CDCl}_{3}\right) \delta 190.8,166.4,163.7,139.0,129.1,129.0$ (x2), 126.4, 115.4, 110.5, 101.1, 80.2, 55.9, 44.5; LRMS (electrospray): Exact mass calcd for $\mathrm{C}_{16} \mathrm{H}_{14} \mathrm{O}_{3}[\mathrm{M}]^{+}, 254.09$. Found [M+1], 255.6.

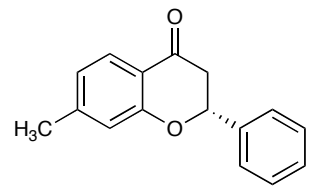

(R)-7-methyl-2-phenylchroman-4-one (14): Prepared according to general procedure using (E)-tert-butyl 2-(2-hydroxy-4methylphenylcarbonyl)-3-phenylprop-2-enoate $(51 \mathrm{mg}, 0.15 \mathrm{mmol})$, thiourea catalyst III $(10 \mathrm{mg}, 0.020 \mathrm{mmol})$ in $1.5 \mathrm{~mL}$ toluene for $6 \mathrm{~d}$ at $-25^{\circ} \mathrm{C}$ and $p$-toluenesulfonic acid $(15 \mathrm{mg}, 0.08 \mathrm{mmol})$ for $9 \mathrm{~h}$. Purification via column chromatography with $10 \%$ EtOAc/hexanes afforded $35 \mathrm{mg}(97 \%)$ of $\mathbf{1 4}$ as a white solid in $90 \%$ ee. $[\alpha]_{\mathrm{D}}:+54.8(\mathrm{EtOH}, \mathrm{c}=0.52)$. Analytical data for 14: IR (film) 1688.6, 1616.6, 1295.2, $1242.3 \mathrm{~cm}^{-1}$; ${ }^{1} \mathrm{H}$ NMR $\left(500 \mathrm{MHz}, \mathrm{CDCl}_{3}\right) \delta 7.83(\mathrm{~d}, J=8.4 \mathrm{~Hz}, 1 \mathrm{H}), 7.49-7.38$ $(\mathrm{m}, 5 \mathrm{H}), 6.89-6.87(\mathrm{~m}, 2 \mathrm{H}), 5.46(\mathrm{dd}, J=13.3,2.7 \mathrm{~Hz}, 1 \mathrm{H}), 3.06(\mathrm{dd}, J=16.9,13.3 \mathrm{~Hz}$, $1 \mathrm{H}), 2.87(\mathrm{dd}, J=16.9,2.7 \mathrm{~Hz}, 1 \mathrm{H}) 2.34(\mathrm{~s}, 3 \mathrm{H}) ;{ }^{13} \mathrm{C} \mathrm{NMR}\left(125 \mathrm{MHz}, \mathrm{CDCl}_{3}\right) \delta$ 192.0, 161.8, 148.0, 139.1, 129.1, 129.0, 127.2, 126.4, 123.2, 118.9, 118.4, 79.8, 44.9, 22.2; LRMS (electrospray): Exact mass calcd for $\mathrm{C}_{16} \mathrm{H}_{14} \mathrm{O}_{2}[\mathrm{M}]^{+}, 238.10$. Found [M+1], 239.1.

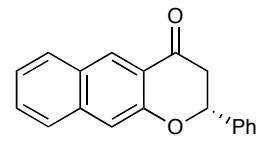

(R)-6,7-benzo-2-phenylchroman-4-one (15): Prepared according to general procedure using (E)-tert-butyl 2-(3-hydroxynaphthalene-2carbonyl)-3-phenylprop-2-enoate $(59 \mathrm{mg}, 0.20 \mathrm{mmol})$, thiourea catalyst III (10 mg, $0.016 \mathrm{mmol})$ in $1.5 \mathrm{~mL}$ toluene for $8 \mathrm{~d}$ at $-25{ }^{\circ} \mathrm{C}$ and $p$-toluenesulfonic acid $(15 \mathrm{mg}, 0.015 \mathrm{mmol}$ ) for $10 \mathrm{~h}$. Purification via column chromatography with $10 \%$ EtOAc/hexanes afforded $34 \mathrm{mg}(78 \%)$ of $\mathbf{1 5}$ as a yellow solid in $89 \%$ ee. $[\alpha]_{\mathrm{D}}:+31.9$ $(\mathrm{EtOH}, \mathrm{c}=0.5)$. Analytical data for 15: IR (film) $1722.1,1639.5,1255.2,1154.6 \mathrm{~cm}^{-1} ;{ }^{1} \mathrm{H}$ NMR $\left(500 \mathrm{MHz} \mathrm{CDCl}_{3}\right) \delta 8.52(\mathrm{~s}, 1 \mathrm{H}) ; 7.88(\mathrm{~d}, J=8.0 \mathrm{~Hz}, 1 \mathrm{H}) ; 7.69(\mathrm{~d}, J=8.5 \mathrm{~Hz}$, 
$1 \mathrm{H})$; 7.52-7.34 (m, 8H), $5.51(\mathrm{~d}, J=12.2 \mathrm{~Hz}, 1 \mathrm{H}), 3.19(\mathrm{dd}, J=17.1,12.8 \mathrm{~Hz}, 1 \mathrm{H}), 3.01$ (dd, $J=17.0,3.0 \mathrm{~Hz}, 1 \mathrm{H}) ;{ }^{13} \mathrm{C}$ NMR $\left(125 \mathrm{MHz}, \mathrm{CDCl}_{3}\right) \delta 192.9,156.9,139.2,138.2$, 130.3, 129.5, 129.4, 129.1, 129.0, 128.8, 127.0, 126.4, 125.1, 121.8, 113.3, 79.4, 45.8; LRMS (electrospray): Exact mass calcd for $\mathrm{C}_{19} \mathrm{H}_{14} \mathrm{O}_{2}[\mathrm{M}]^{+}$, 274.10. Found [M+1], 275.2.

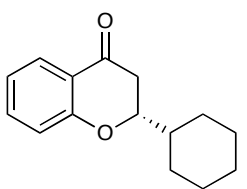

(R)-2-(cyclohexyl)chroman-4-one (16): Prepared according to general procedure using (E)-tert-butyl 3-cyclohexyl-2-(2hydroxyphenylcarbonyl)prop-2-enoate $(66 \mathrm{mg}, 0.20 \mathrm{mmol})$, thiourea catalyst III $(14 \mathrm{mg}, 0.020 \mathrm{mmol})$ in $2.0 \mathrm{~mL}$ toluene for $5 \mathrm{~d}$ at $-25^{\circ} \mathrm{C}$ and $p$-toluenesulfonic acid $(19 \mathrm{mg}, 0.10 \mathrm{mmol})$ for $4 \mathrm{~h}$. Purification via column chromatography with $10 \%$ EtOAc/hexanes afforded $30.0 \mathrm{mg}(65 \%)$ of $\mathbf{1 6}$ as a white solid in $80 \%$ ee. $[\alpha]_{\mathrm{D}}:-53.0(\mathrm{EtOH}, \mathrm{c}=0.5)$. Analytical data for 16: IR (film) 2928.8, 1689.6, 1608.8, 1467.1, 1310.1, $1229.6 \mathrm{~cm}^{-1} ;{ }^{1} \mathrm{H}$ NMR (500 MHz, $\left.\mathrm{CDCl}_{3}\right) \delta 7.87$ (d, $J=7.3 \mathrm{~Hz}, 1 \mathrm{H}), 7.47(\mathrm{t}, J=7.1 \mathrm{~Hz}, 1 \mathrm{H}), 7.01-6.97(\mathrm{~m}, 2 \mathrm{H}), 4.23-4.19(\mathrm{~m}, 1 \mathrm{H}), 2.77-$ $2.65(\mathrm{~m}, 2 \mathrm{H}), 2.01-1.99(\mathrm{~m}, 1 \mathrm{H}), 1.84-1.72(\mathrm{~m}, 5 \mathrm{H}), 1.35-1.11(\mathrm{~m}, 5 \mathrm{H}) ;{ }^{13} \mathrm{C}$ NMR $(125$ $\mathrm{MHz}, \mathrm{CDCl}_{3}$ ) $\delta 193.4,162.1,136.1,127.1,121.2$ (x2), 118.1, 82.2, 42.0, 40.4, 28.5, 28.4, 26.5, 26.15, 26.09; LRMS (electrospray): Exact mass calcd for $\mathrm{C}_{15} \mathrm{H}_{18} \mathrm{O}_{2}[\mathrm{M}]^{+}, 230.13$. Found [M+1], 231.7.

One-Pot Knoevenagel/Cyclization: To a $25 \mathrm{~mL}$ RBF was added tert-Butyl 3-(2hydroxyphenyl)-3-oxopropanoate $\mathbf{1 8}(71 \mathrm{mg}, 0.3 \mathrm{mmol})$, thiourea catalyst I, (36 $\mathrm{mg}, 20$ mol\%), and $4 \AA \mathrm{MS}(0.50 \mathrm{~g})$. The flask was purged with $\mathrm{N}_{2}$. Toluene $(9.0 \mathrm{~mL})$, hydrocinnamaldehyde $(40 \mu \mathrm{L}, 0.3 \mathrm{mmol})$ and piperidinium acetate $(0.025 \mathrm{M}$ in toluene, $0.6 \mathrm{~mL}$ ) were added and the heterogenous mixture was stirred at $22{ }^{\circ} \mathrm{C}$ for $2 \mathrm{~d}$. The reaction was taken up in EtOAc $(40 \mathrm{~mL})$ and washed with brine $(25 \mathrm{~mL})$. The organic layer was dried with $\mathrm{Na}_{2} \mathrm{SO}_{4}$, filtered concentrated to give $93 \mathrm{mg}$ of a pink oil (88\%) which was decarboxylated with $6.0 \mathrm{~mL}$ toluene, $15 \mathrm{mg} p$-TsOH, heating to $80{ }^{\circ} \mathrm{C}$ for $9 \mathrm{~h}$. The reaction was taken up in EtOAc $(30 \mathrm{~mL})$ and washed with brine $(15 \mathrm{~mL})$. The organic layer was dried with $\mathrm{Na}_{2} \mathrm{SO}_{4}$, filtered, concentrated. Purification via column chromatography with $10 \%$ EtOAc/hexanes afforded $57 \mathrm{mg} 20$ as a yellow solid (88\%) in $80 \%$ ee. $[\alpha]_{D}:-59.0(\mathrm{EtOH}, \mathrm{c}=0.62)$.

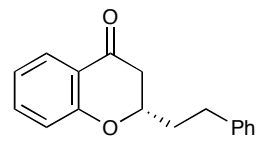

(S)-2-Phenethylchroman-4-one

((S)-Flindersiachromanone)

(20). Analytical data match those reported in the literature. ${ }^{7}$ Previously unreported ${ }^{13} \mathrm{C}$ NMR $\left(125 \mathrm{MHz}, \mathrm{CDCl}_{3}\right) \delta 192.5,161.8,141.2,136.3,128.8,128.7,127.2,126.4,121.6,121.3$, $118.2,77.0,43.3,36.8,31.4$.

General Procedure for Racemic Cyclizations for HPLC Traces: To a $10 \mathrm{~mL}$ round bottom flask containing a magnetic stirring bar was added alkylidene compound 0.1 mmol, $30 \mathrm{~mol} \%$ 1,8-diazabicyclo[5.4.0]undec-7-ene and $2 \mathrm{~mL}$ acetonitrile. The solution is stirred at $23{ }^{\circ} \mathrm{C}$ for 3-12 h. Reaction progress was monitored by TLC. After complete

\footnotetext{
${ }^{7}$ Kawasaki, M.; Yoshikai, H.; Kakuda, H.; Toyooka, N.; Tanaka, A.; Goto, M.; Kometani, T. Heterocycles 2006, 68, 483-493.
} 
cyclization, $50 \mathrm{~mol} \%$-toluenesulfonic acid was added to the flask and the solution was heated to $80{ }^{\circ} \mathrm{C}$, and the reaction was monitored by TLC. Upon completion of the reaction, the solution was allowed to cool and the solution was diluted with EtOAc (20 $\mathrm{mL}$ ), poured into a separatory funnel and washed with brine. The organic layer was dried over anhydrous $\mathrm{Na}_{2} \mathrm{SO}_{4}$, filtered and concentrated to provide the crude flavanone. The crude mixture was purified via silica gel chromatography (10\% EtOAc/hexanes) and concentrated in vacuo. 


\section{Selected NMR Spectra}

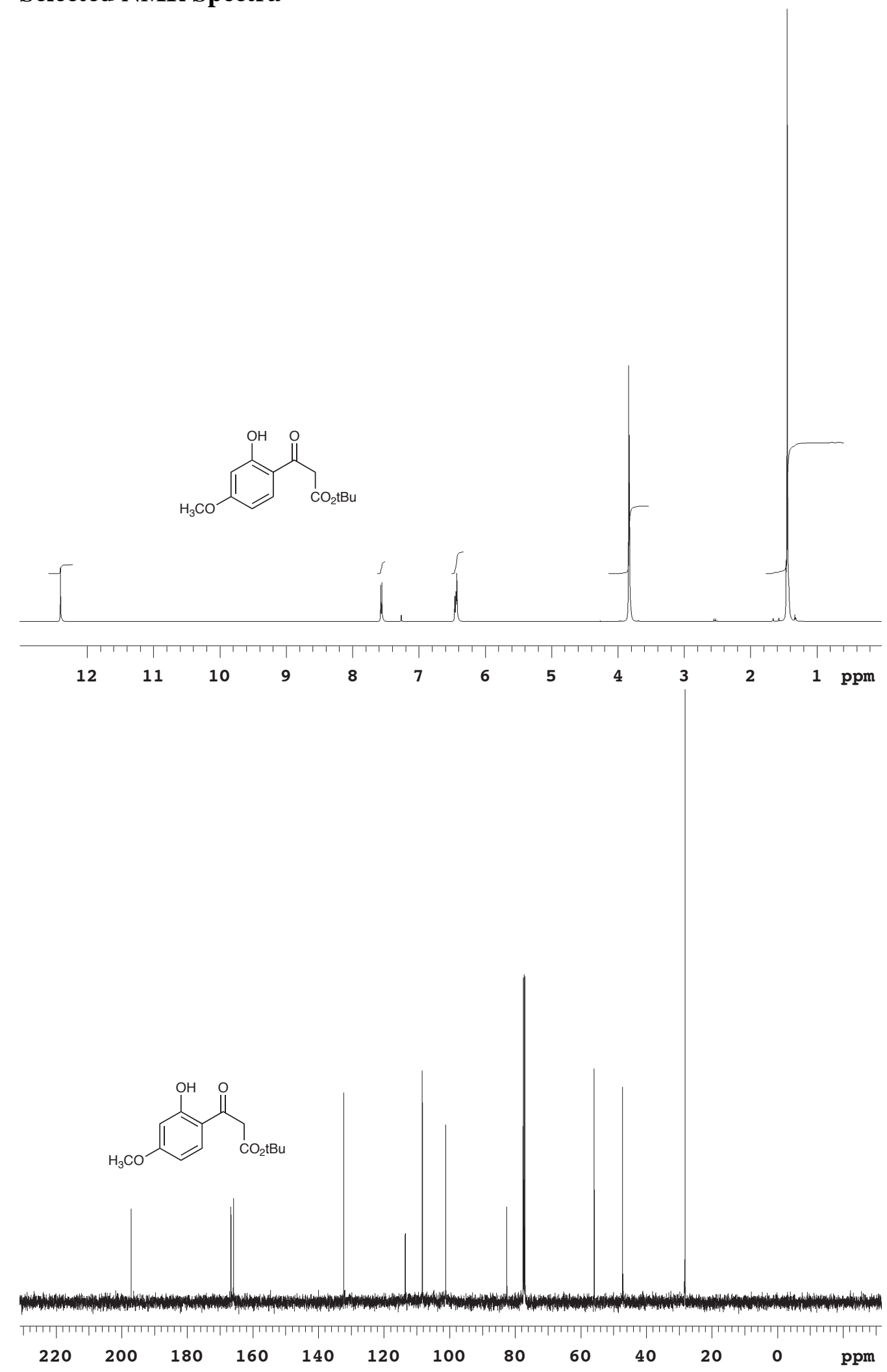




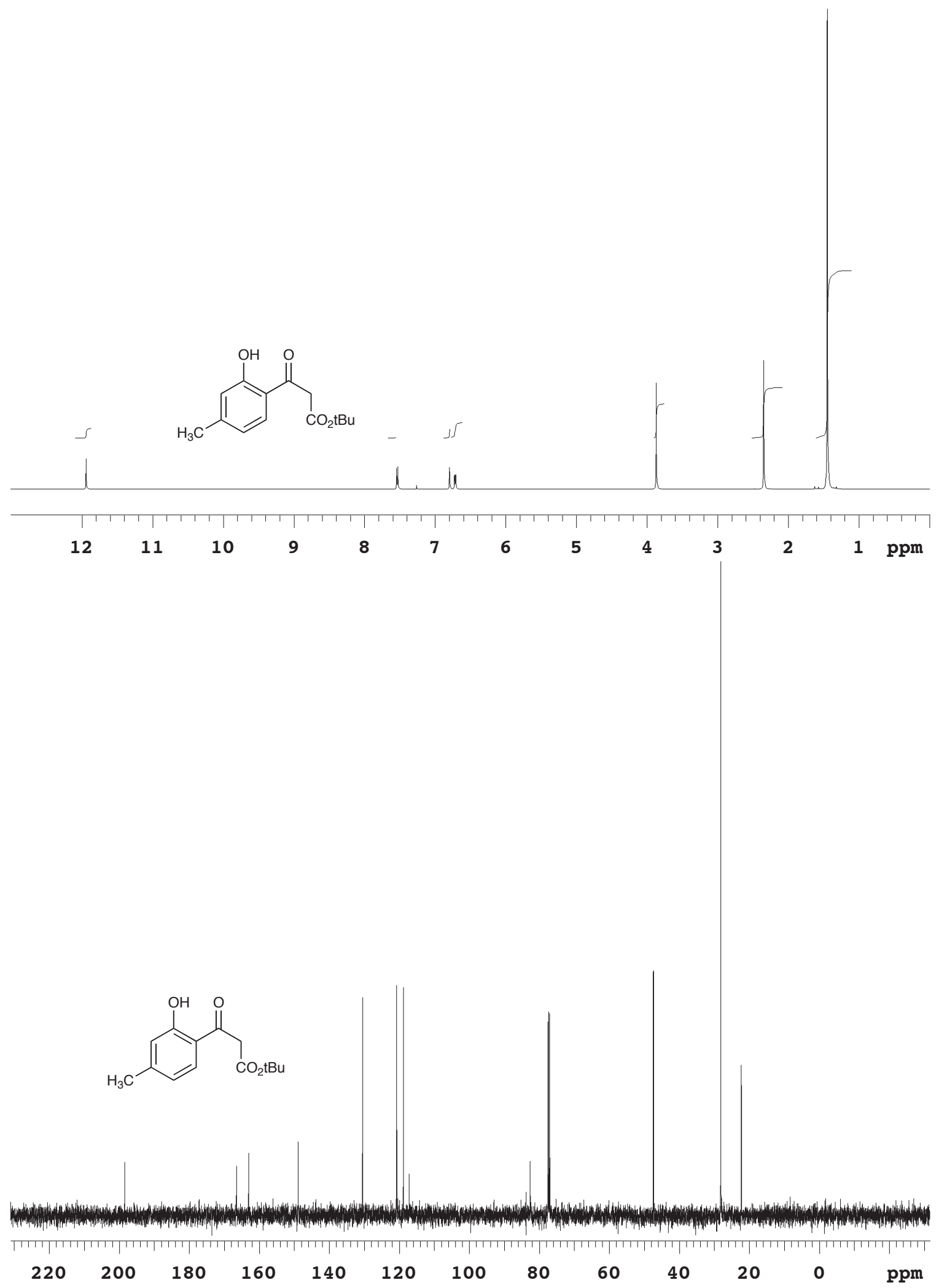




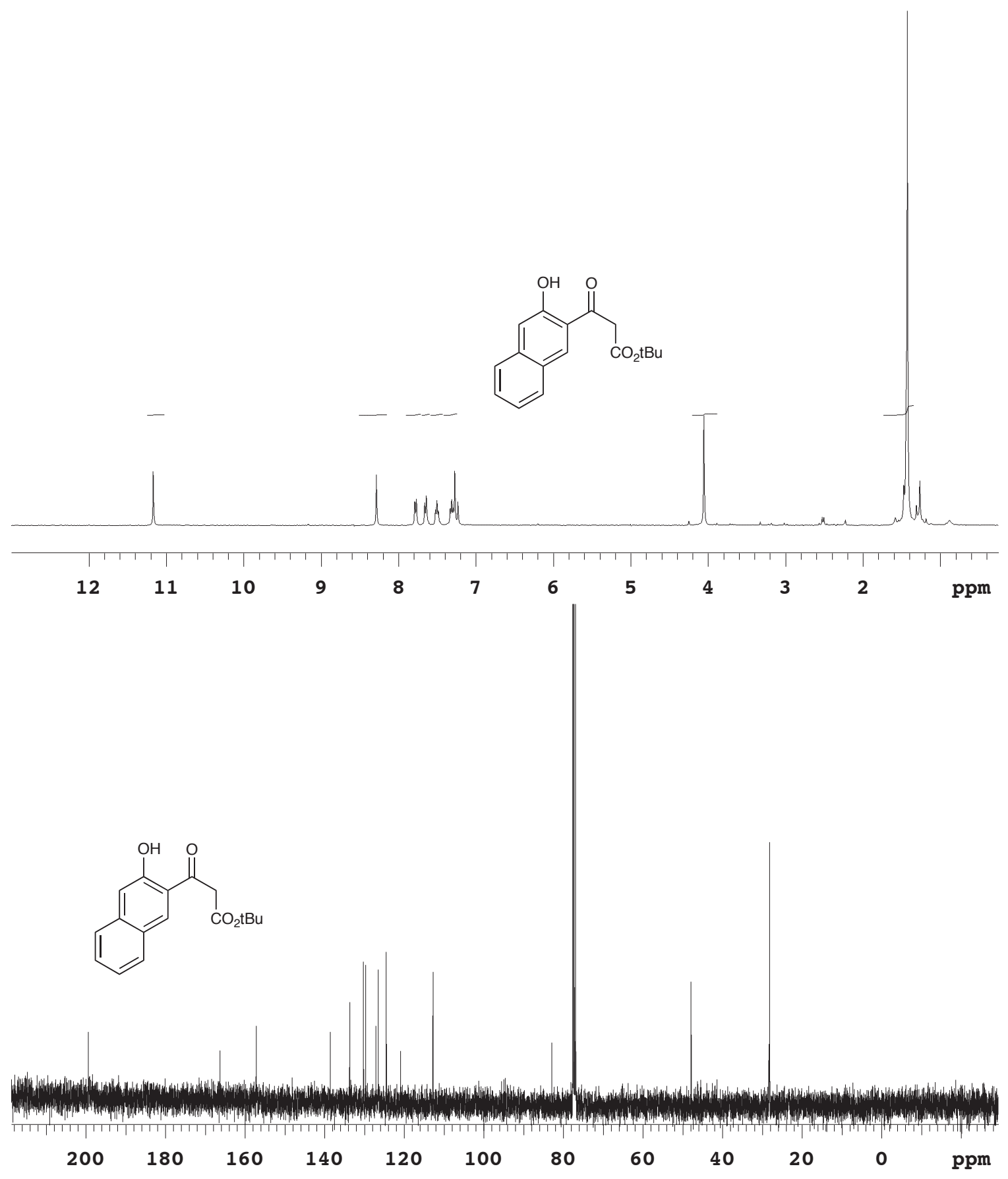




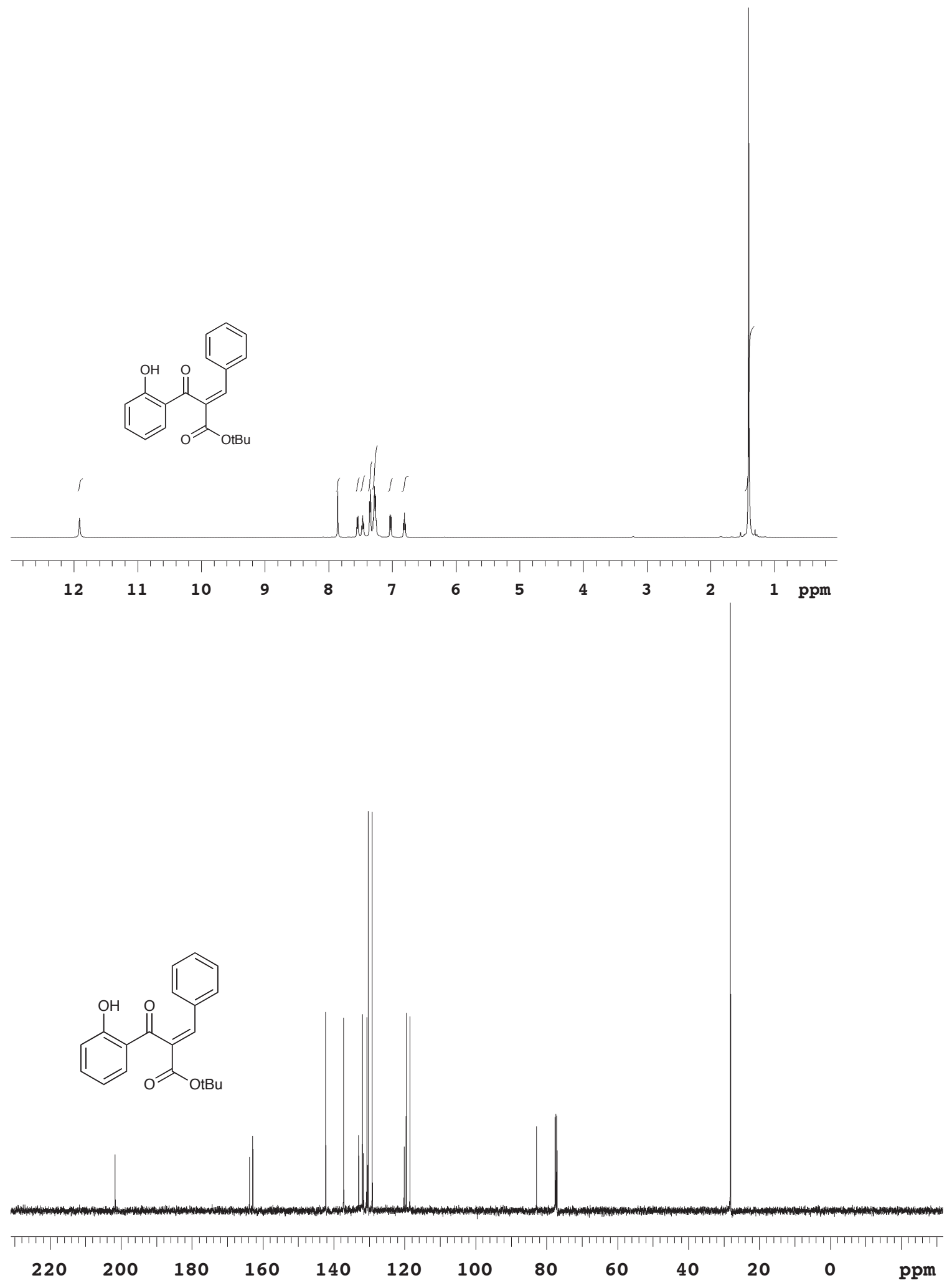




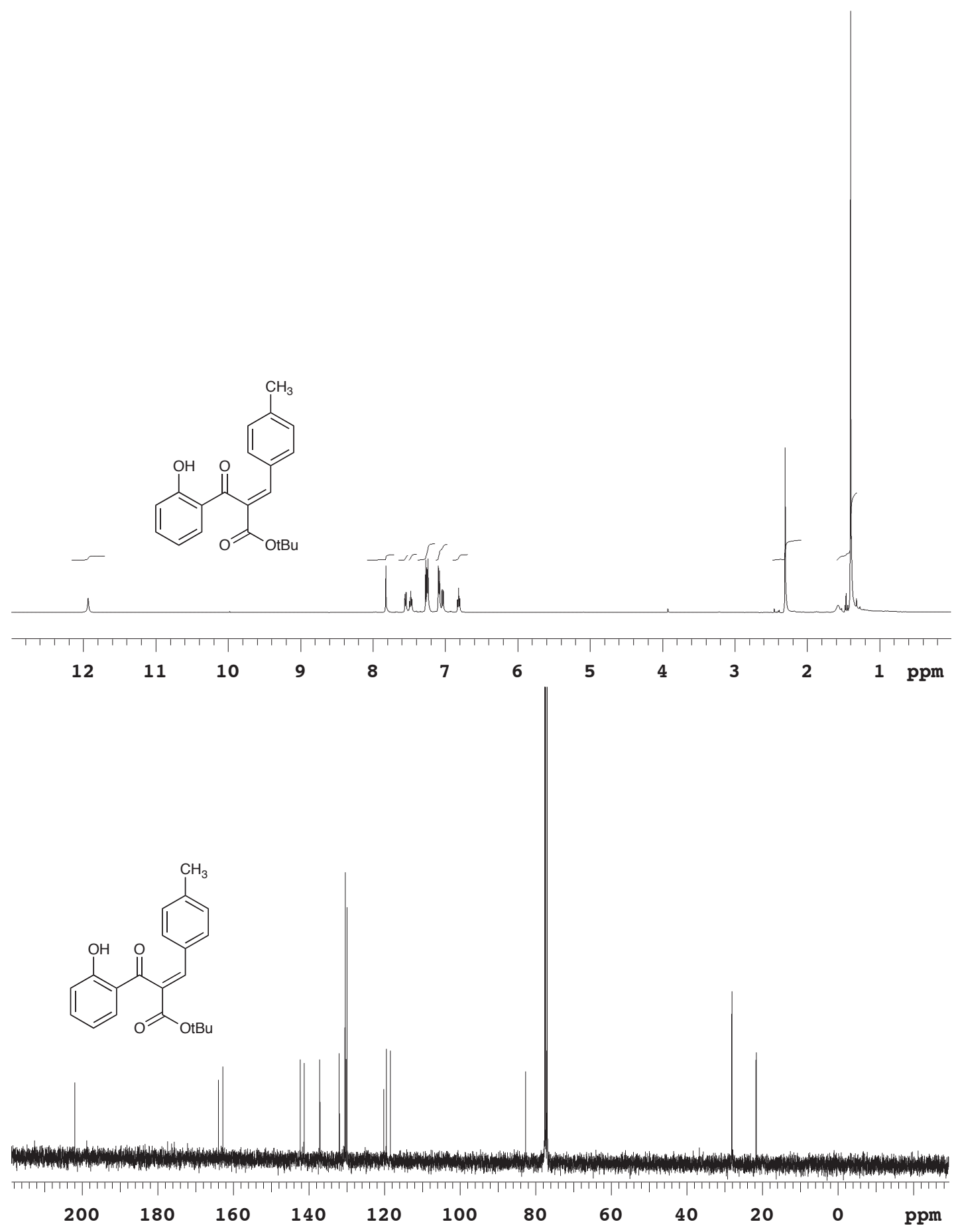




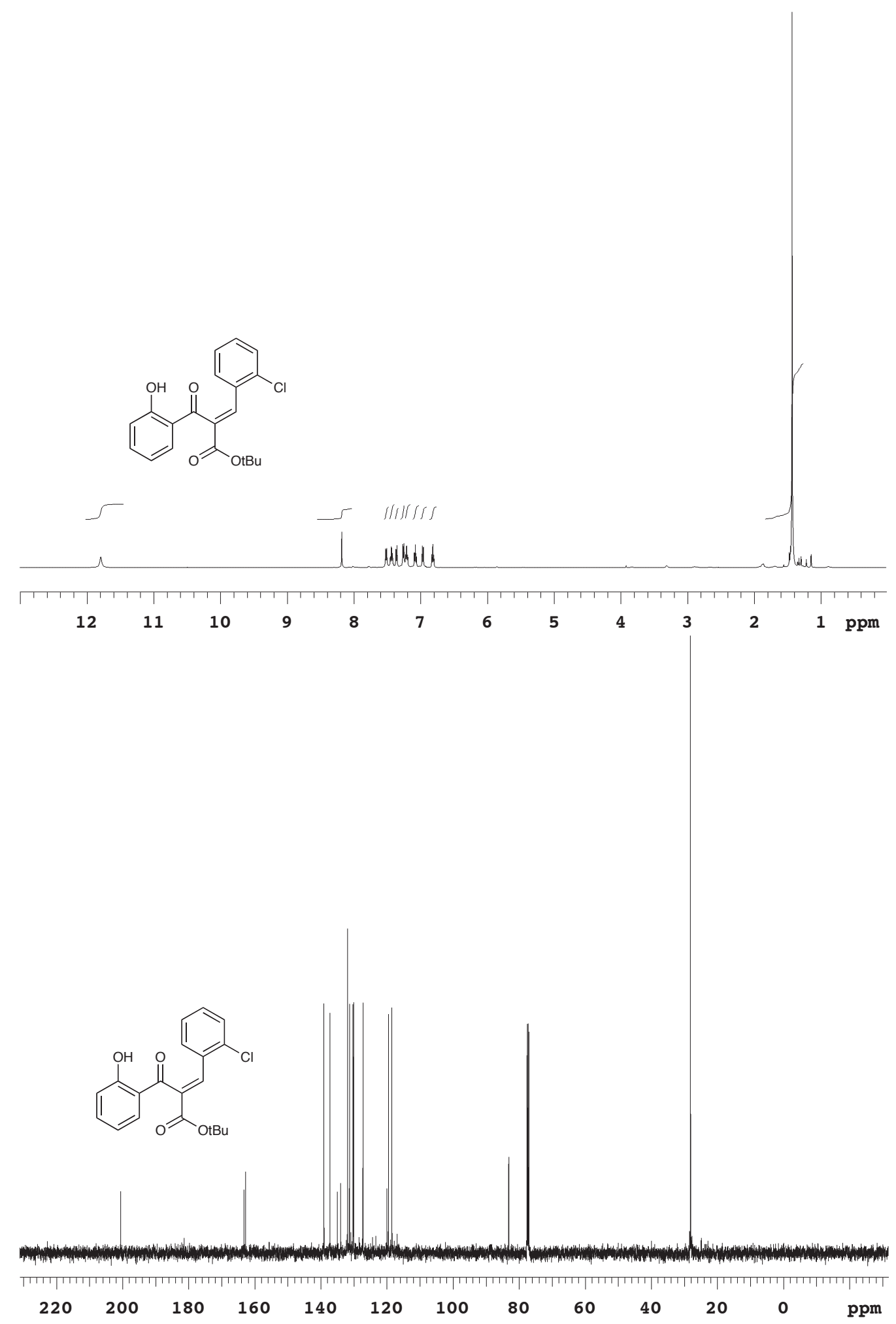



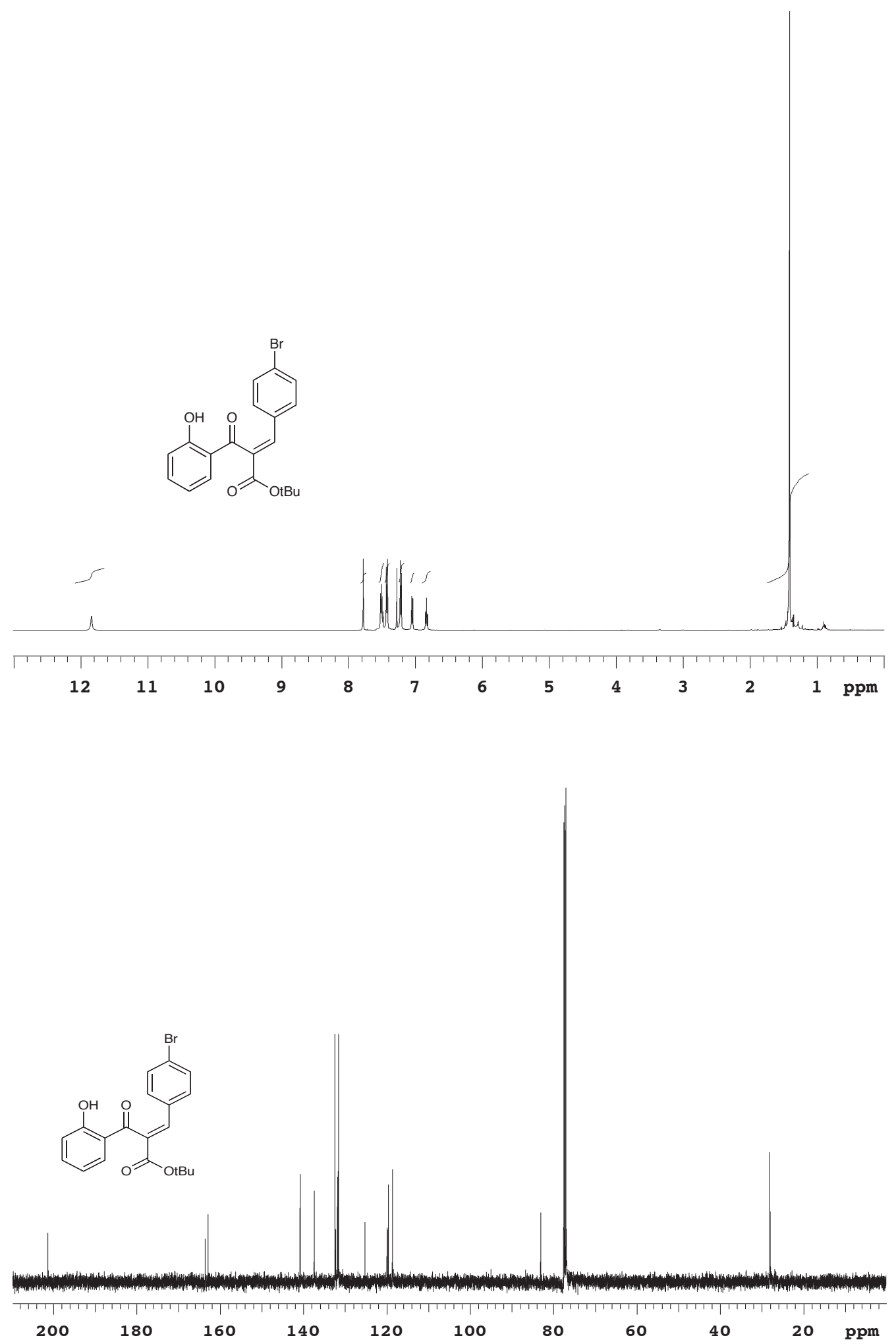

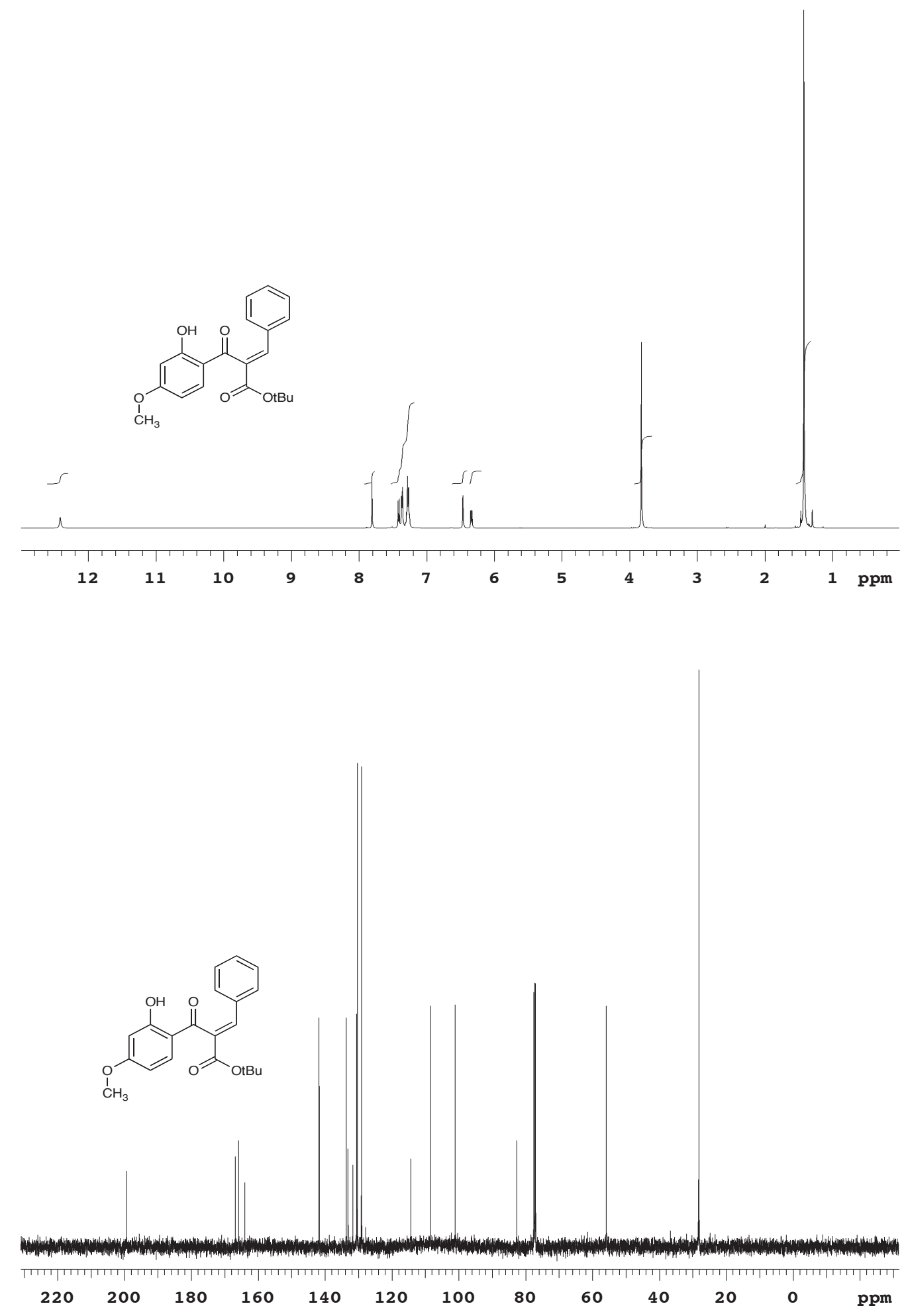

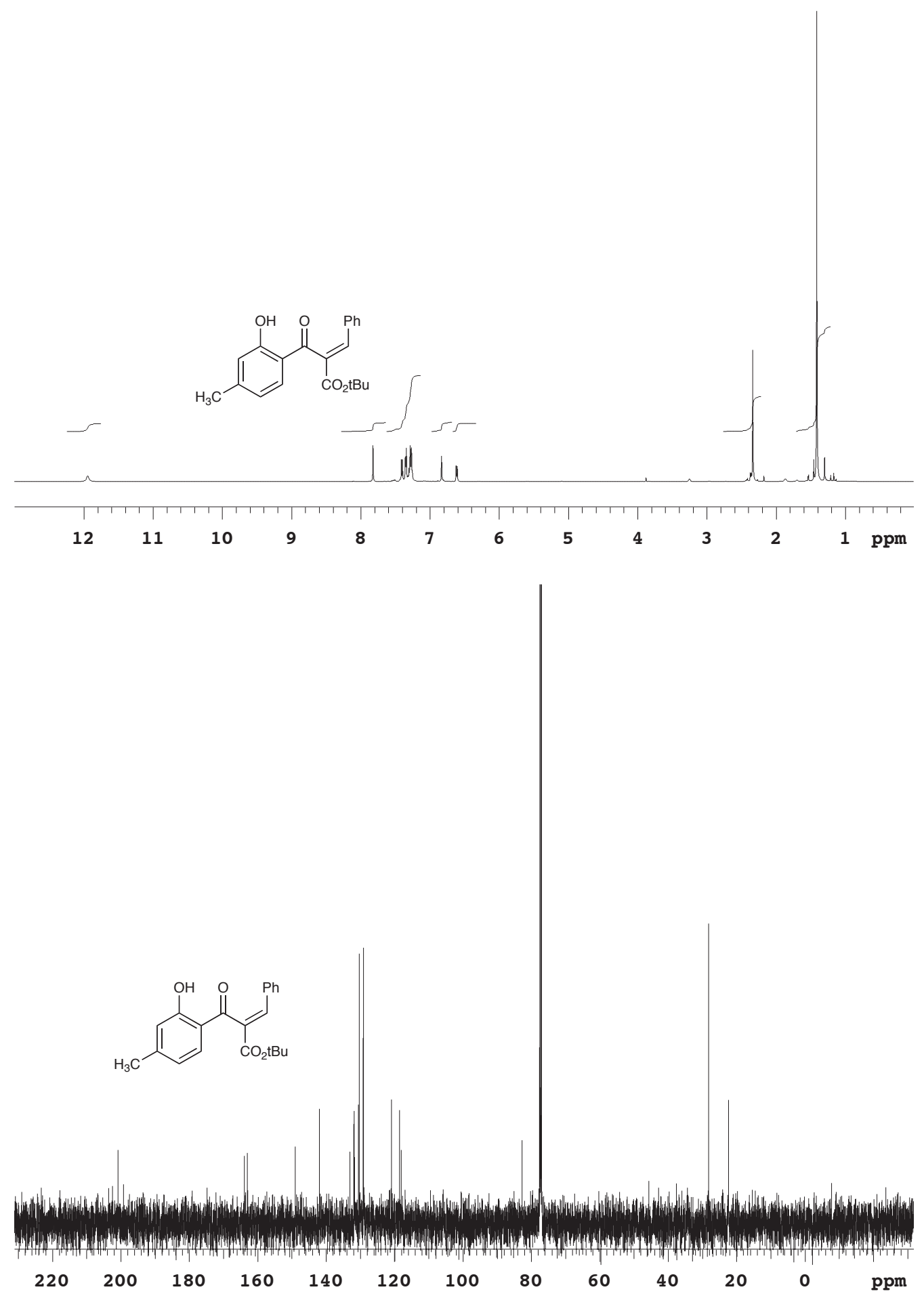

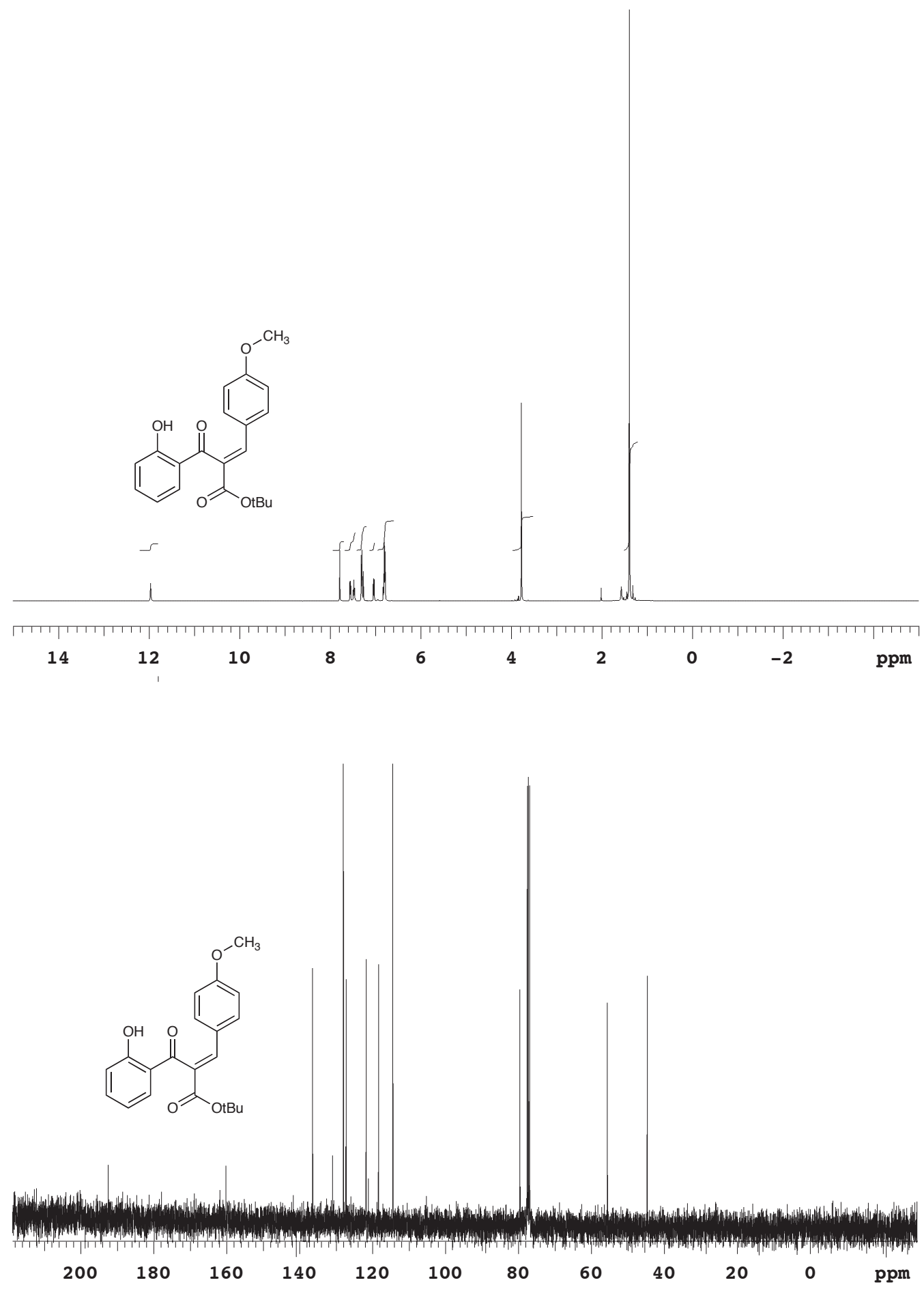


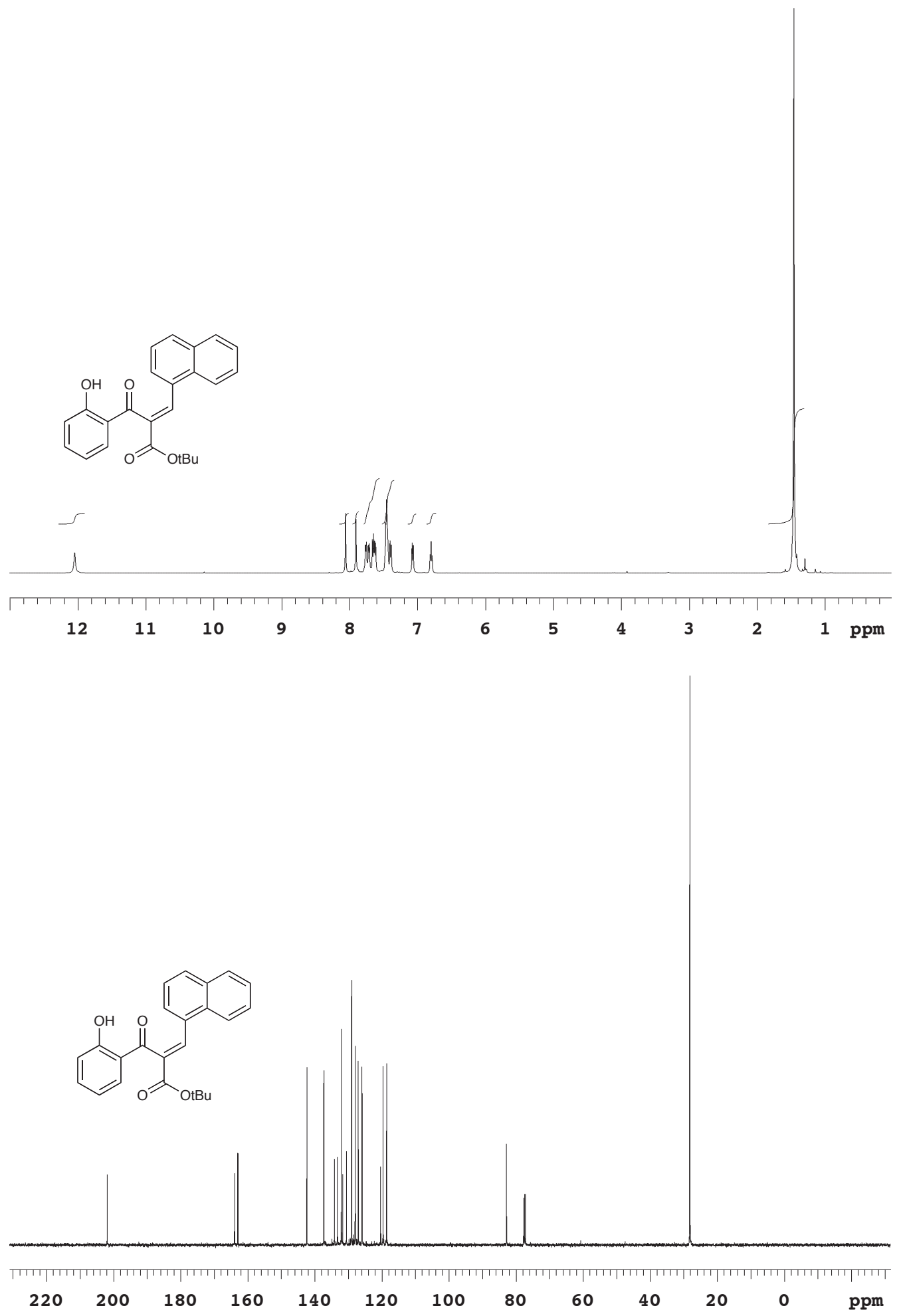




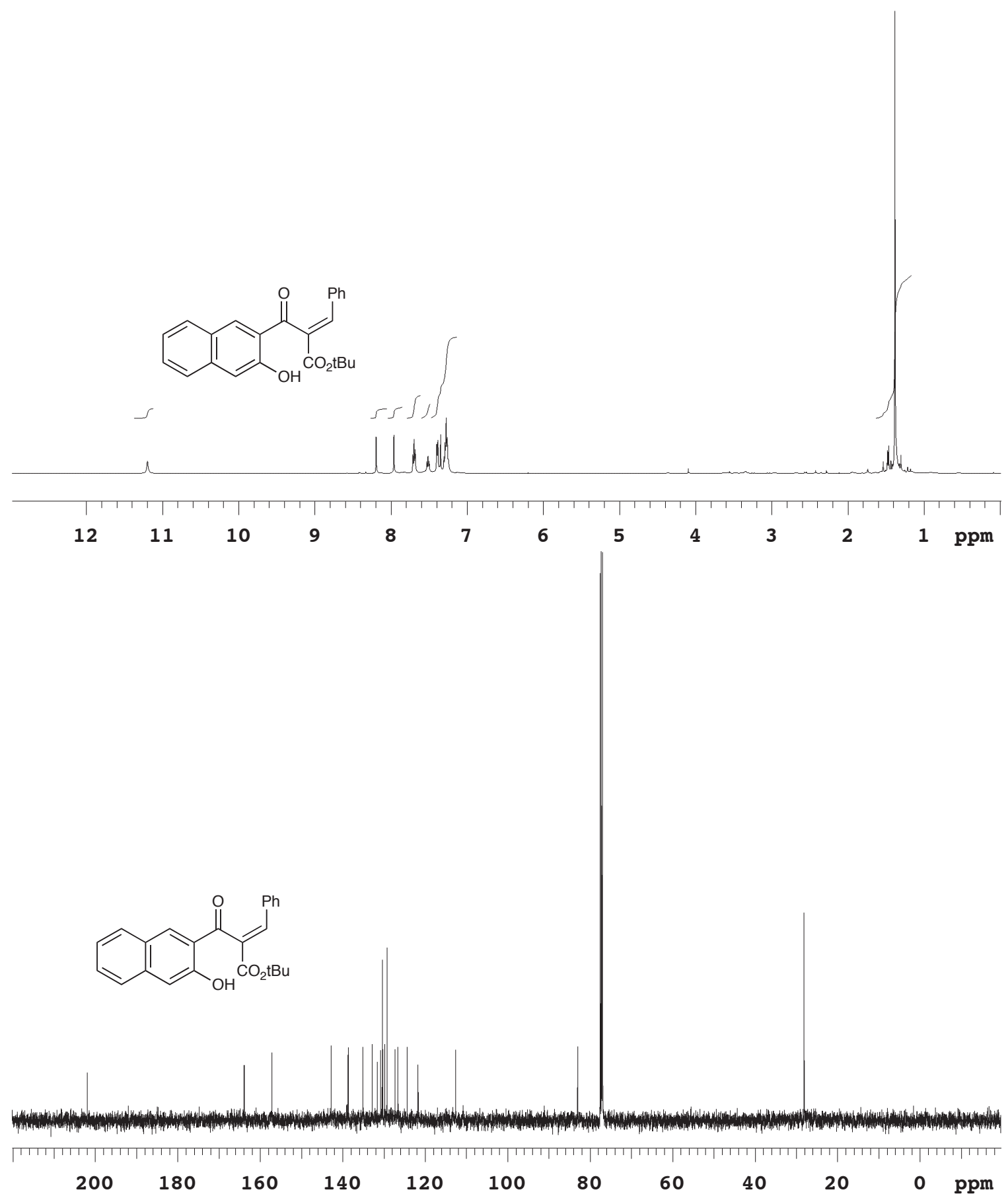




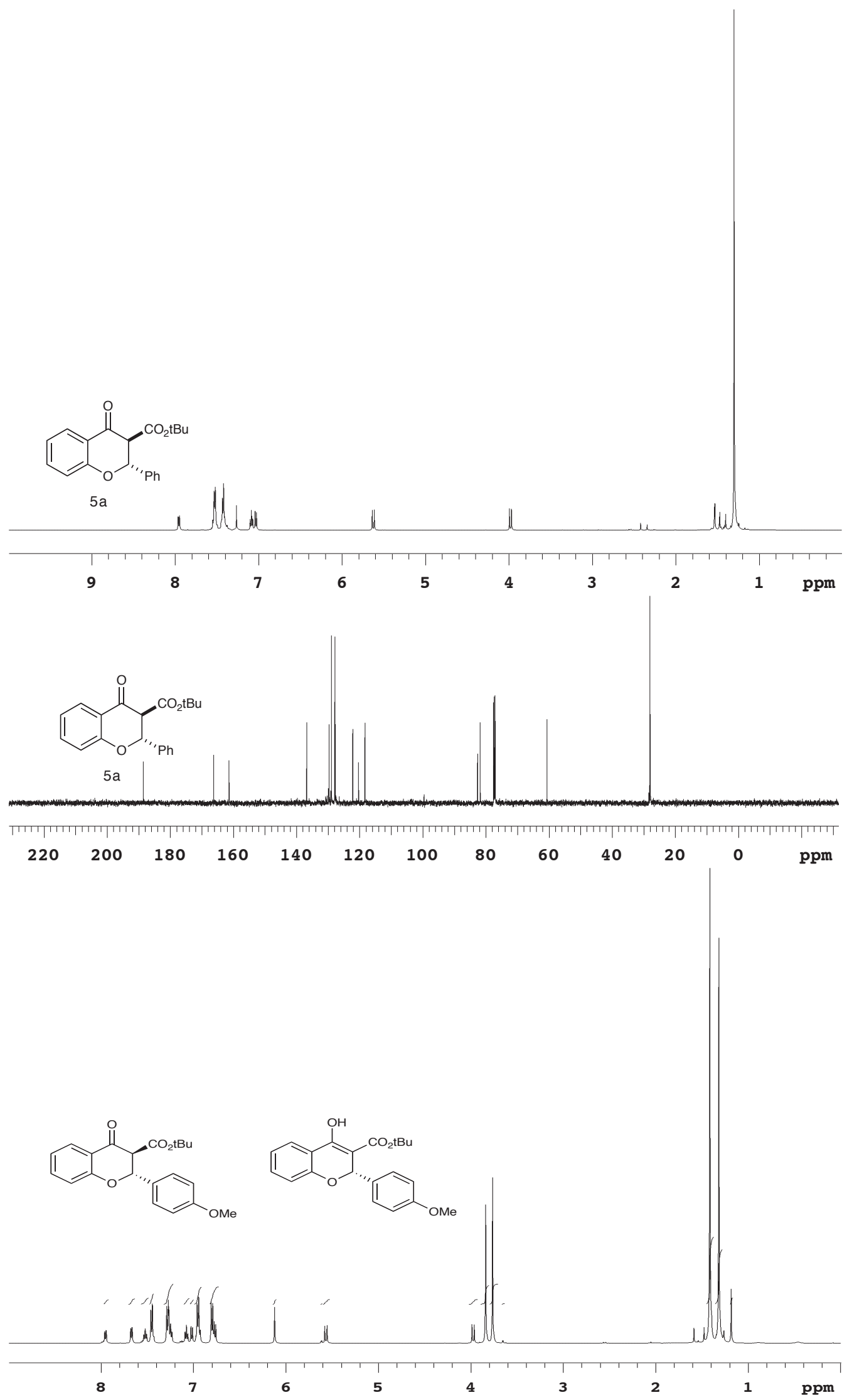




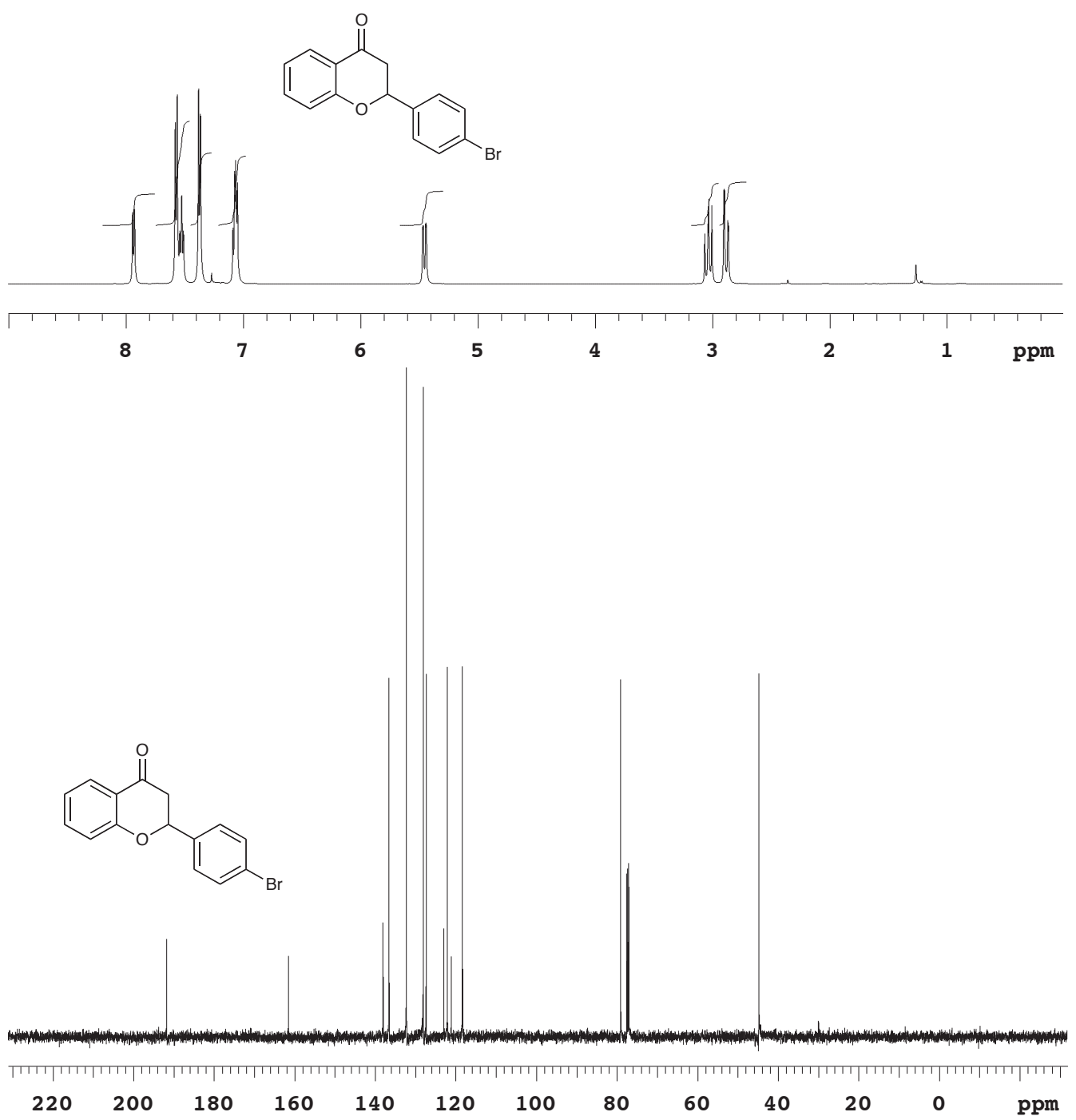



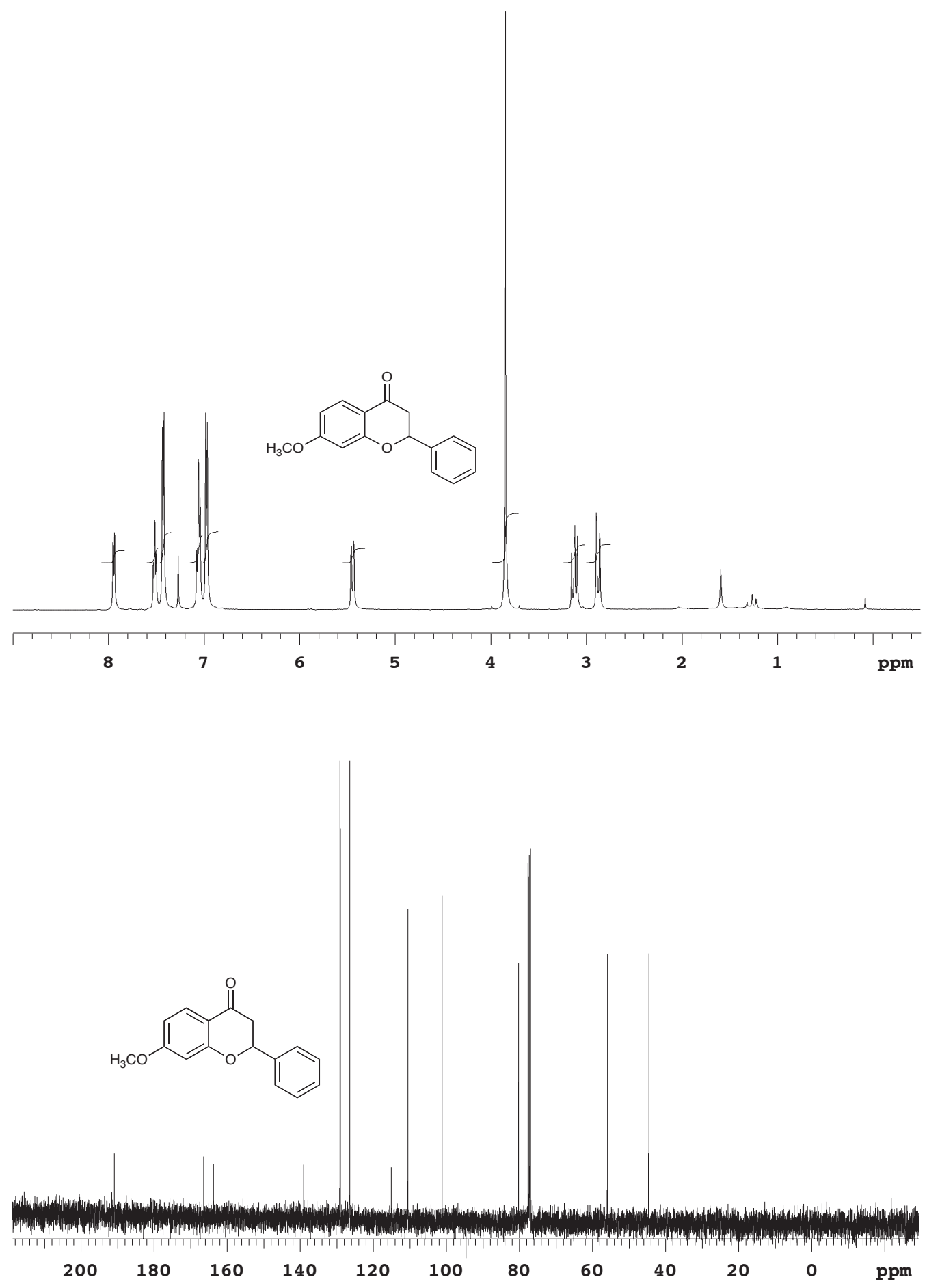

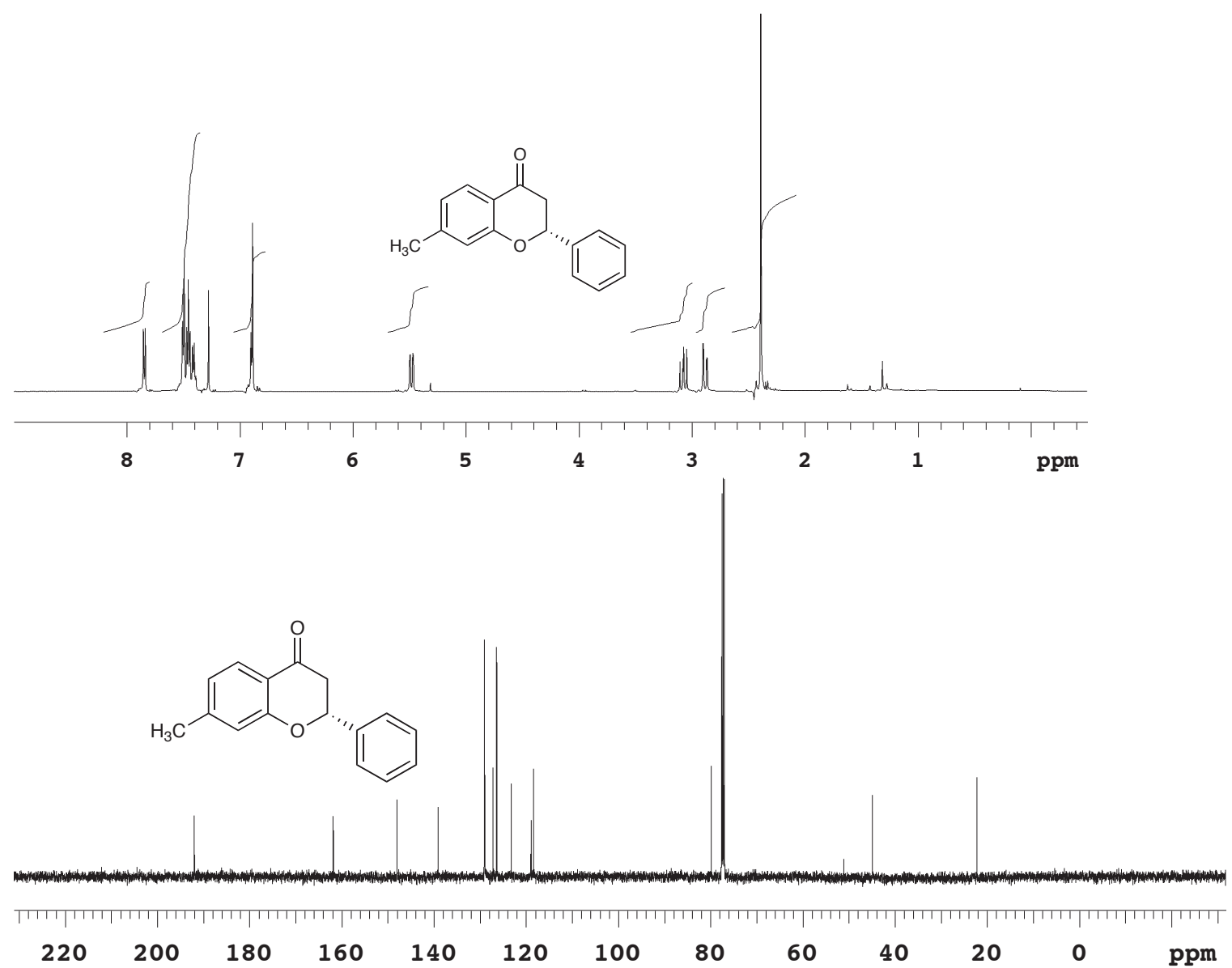


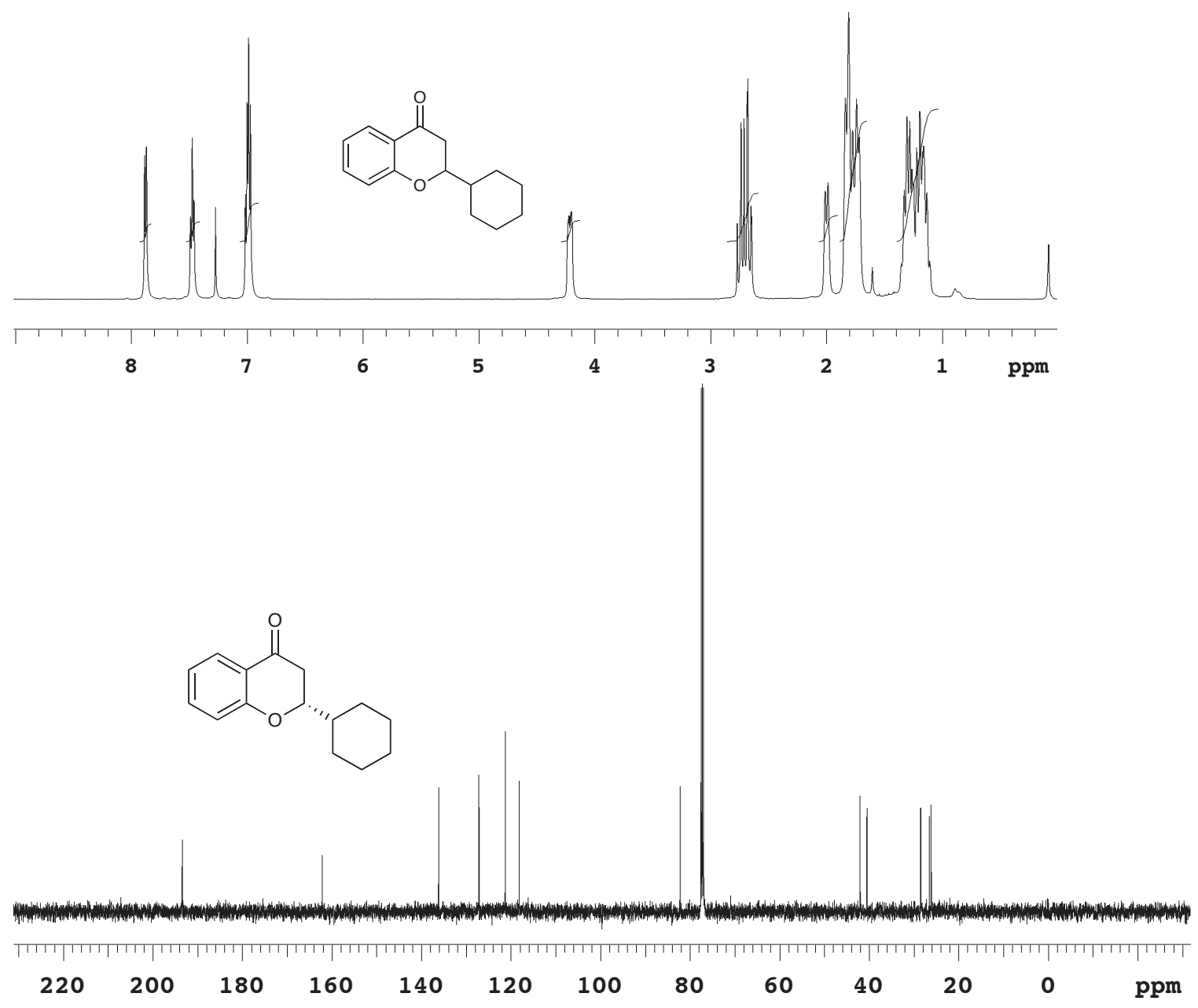




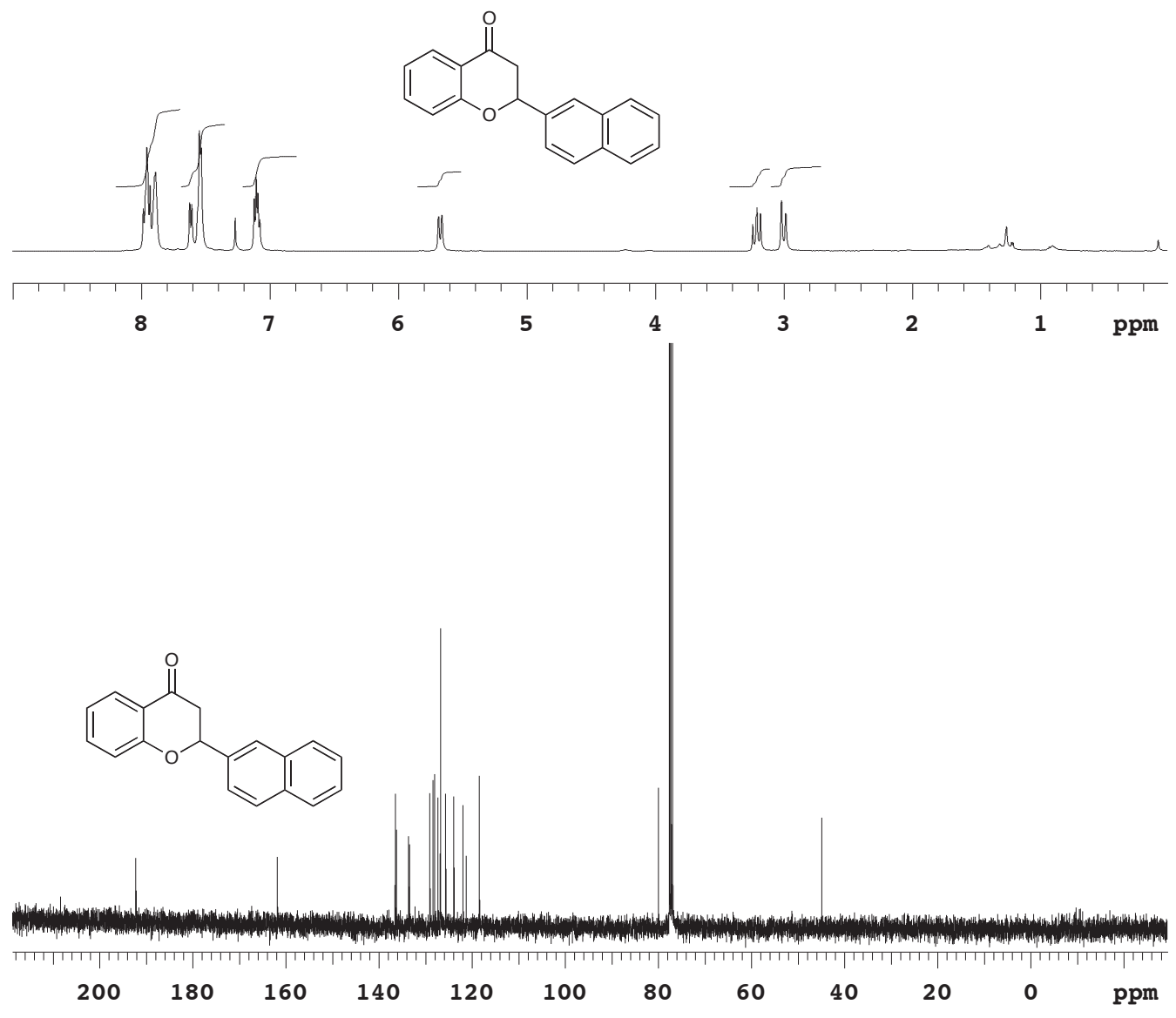



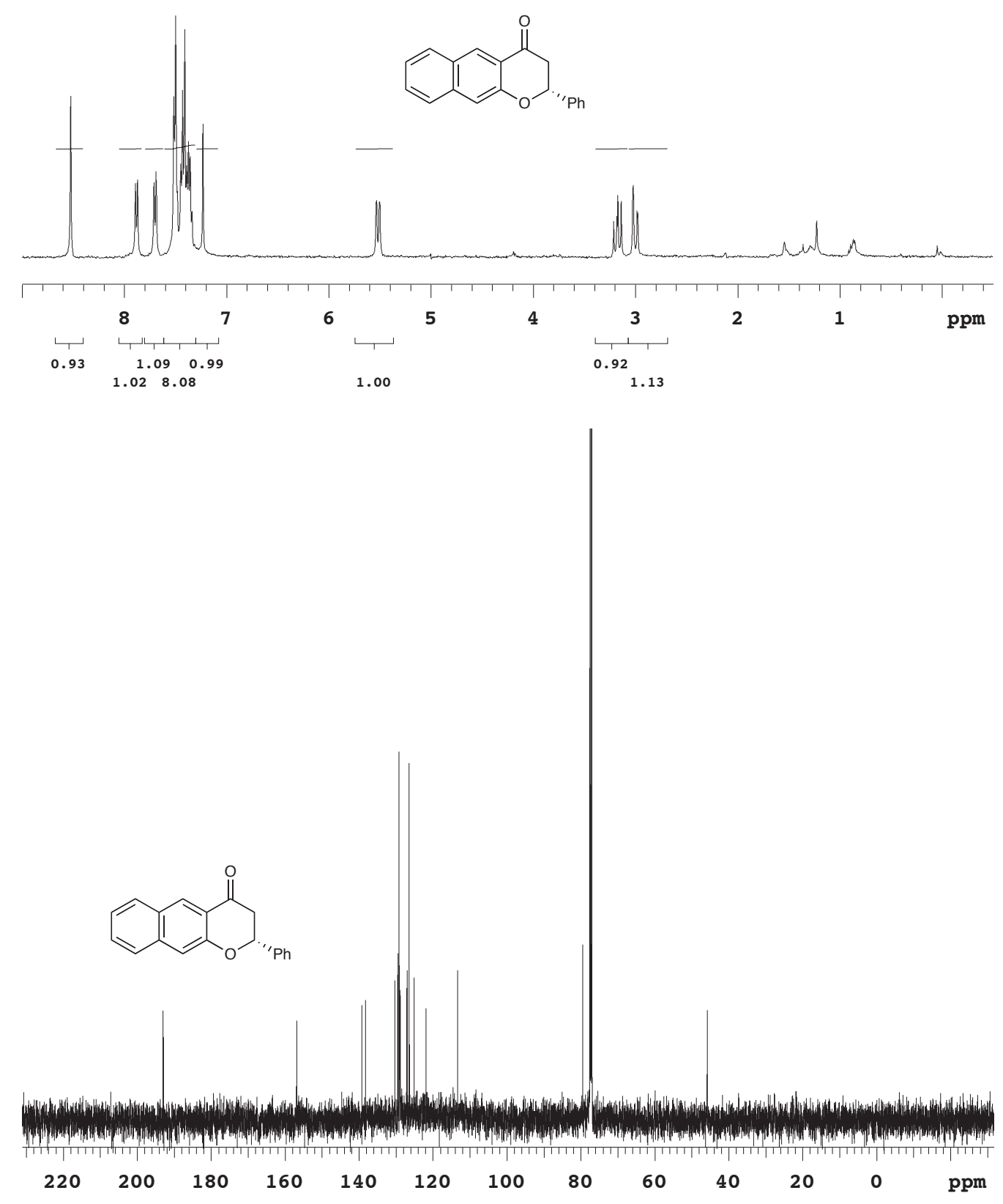

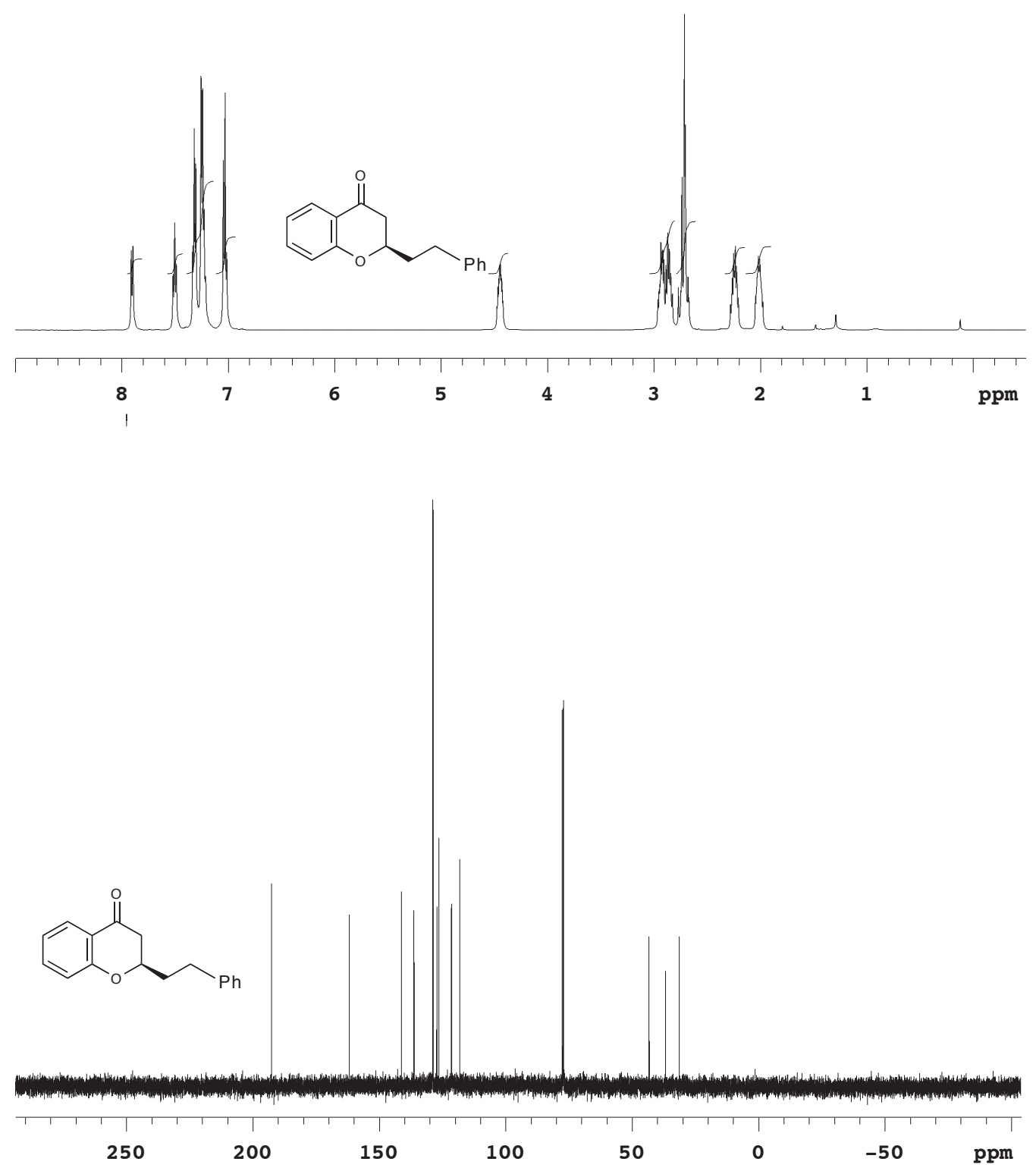


\section{HPLC Traces}

\section{Racemic flavanone (7):}

HPLC Conditions: 95:5 hexanes:isopropanol, $1.0 \mathrm{~mL} / \mathrm{min}$, OD-H Chiralcel column Data File C: $\backslash$ HPCHEM $\backslash \backslash \backslash$ DATA $\backslash M M B \backslash M M P H F O 00 . D$

PhFlav Rac

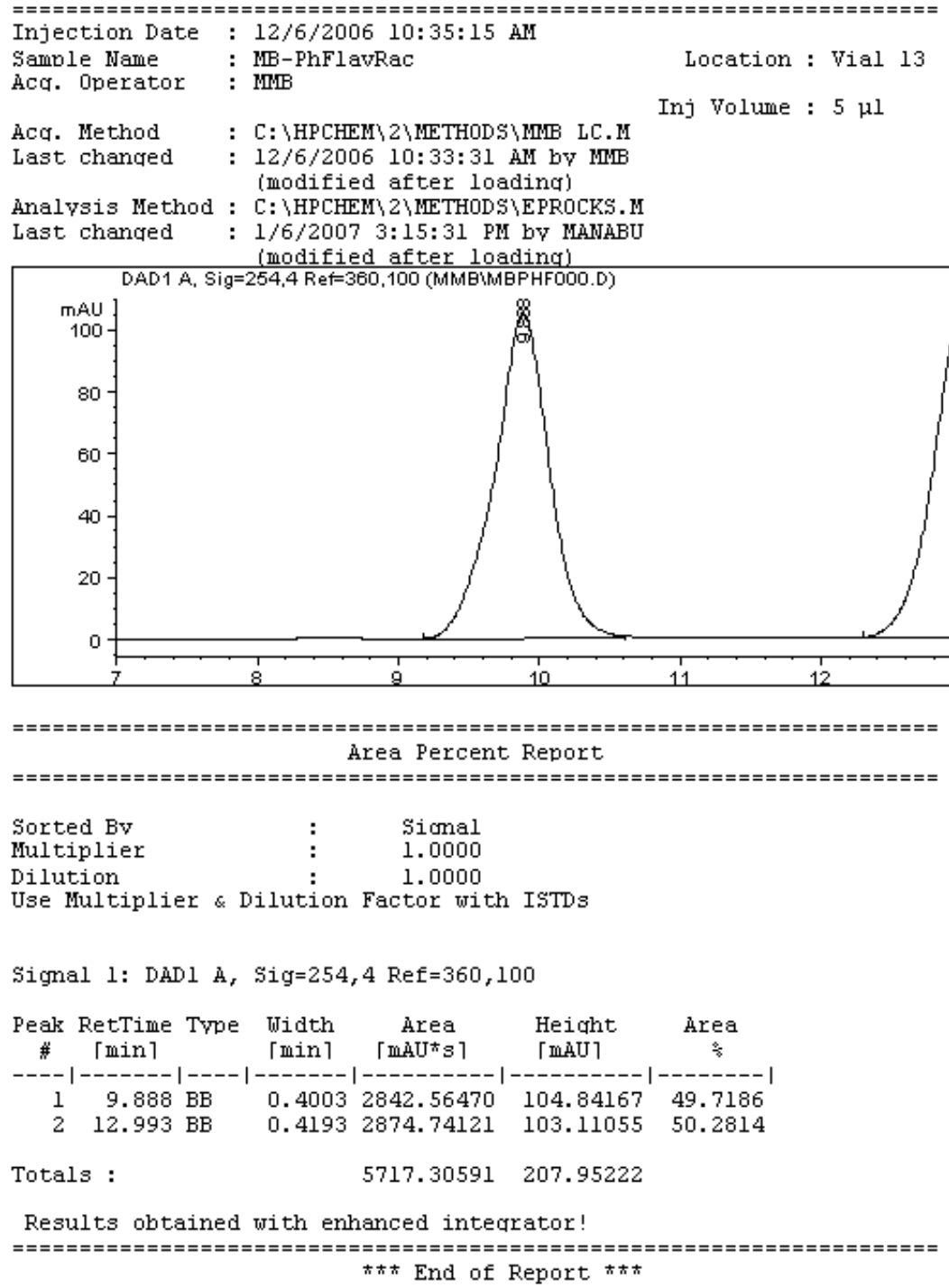

\#ネ End of Report 
Enantioenriched $(R)$-flavanone (7):

HPLC Conditions: 95:5 hexanes:isopropanol, $1.0 \mathrm{~mL} / \mathrm{min}$, OD-H Chiralcel column Data File C: \HPCHEM $2 \backslash D A T A \backslash M \mathbb{M B} \backslash \mathbb{M B} 309400 . \mathrm{D}$

$\mathrm{Ph}$

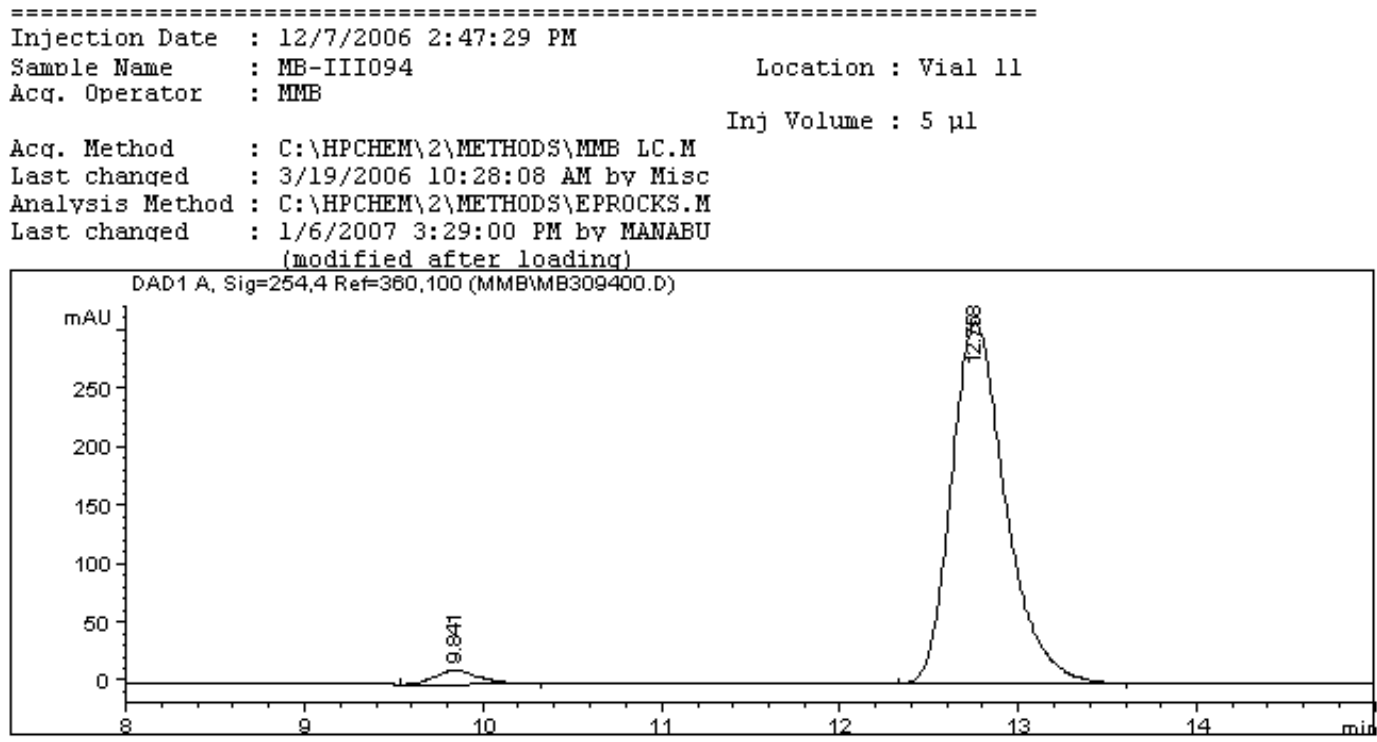

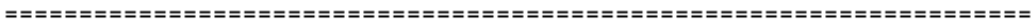

Area Percent Report

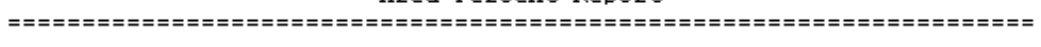

Sorted By $\quad$ : Sicmal

Multivlier : : 1.0000

Dilution : 1.0000

Use Multiplier \& Dilution Factor with IsTDs

Signal 1: DADl À, Sig $=254,4$ Ref $=360,100$

\begin{tabular}{|c|c|c|c|c|c|}
\hline $\begin{array}{c}\text { Peak } \\
\#\end{array}$ & $\begin{array}{l}\text { RetTime Trpe } \\
\text { 「min } 1\end{array}$ & $\begin{array}{l}\text { Width } \\
\text { 「min }\rceil\end{array}$ & 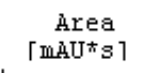 & $\begin{array}{l}\text { Height } \\
\text { 「mAU }\end{array}$ & $\underset{\substack{\text { Area } \\
\frac{\phi}{\delta}}}{ }$ \\
\hline$\frac{1}{2}$ & $\begin{array}{r}9.841 \mathrm{~PB} \\
12.758 \mathrm{BB}\end{array}$ & $\begin{array}{l}0.2670 \\
0.3216\end{array}$ & $\begin{array}{r}194.06070 \\
6521.44189\end{array}$ & $\begin{array}{r}11.15294 \\
309.75095\end{array}$ & $\begin{array}{r}2.8897 \\
97.1103\end{array}$ \\
\hline 01 & & & 6715.50259 & 320.90388 & \\
\hline
\end{tabular}

Results obtained with enhanced integrator!

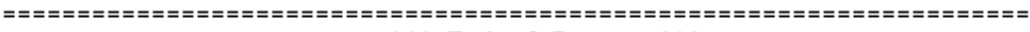

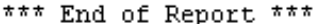




\section{Racemic 2-(4-bromophenyl)chroman-4-one (8):}

HPLC Conditions: 95:5 hexanes:isopropanol, $1.0 \mathrm{~mL} / \mathrm{min}, \mathrm{OD}-\mathrm{H}$ Chiralcel column Data File C: \HPCHEM $2 \backslash D A T A \backslash M \mathbb{M} \backslash \mathbb{M B}-4 B R F 0 . D$

4BrFlavRac

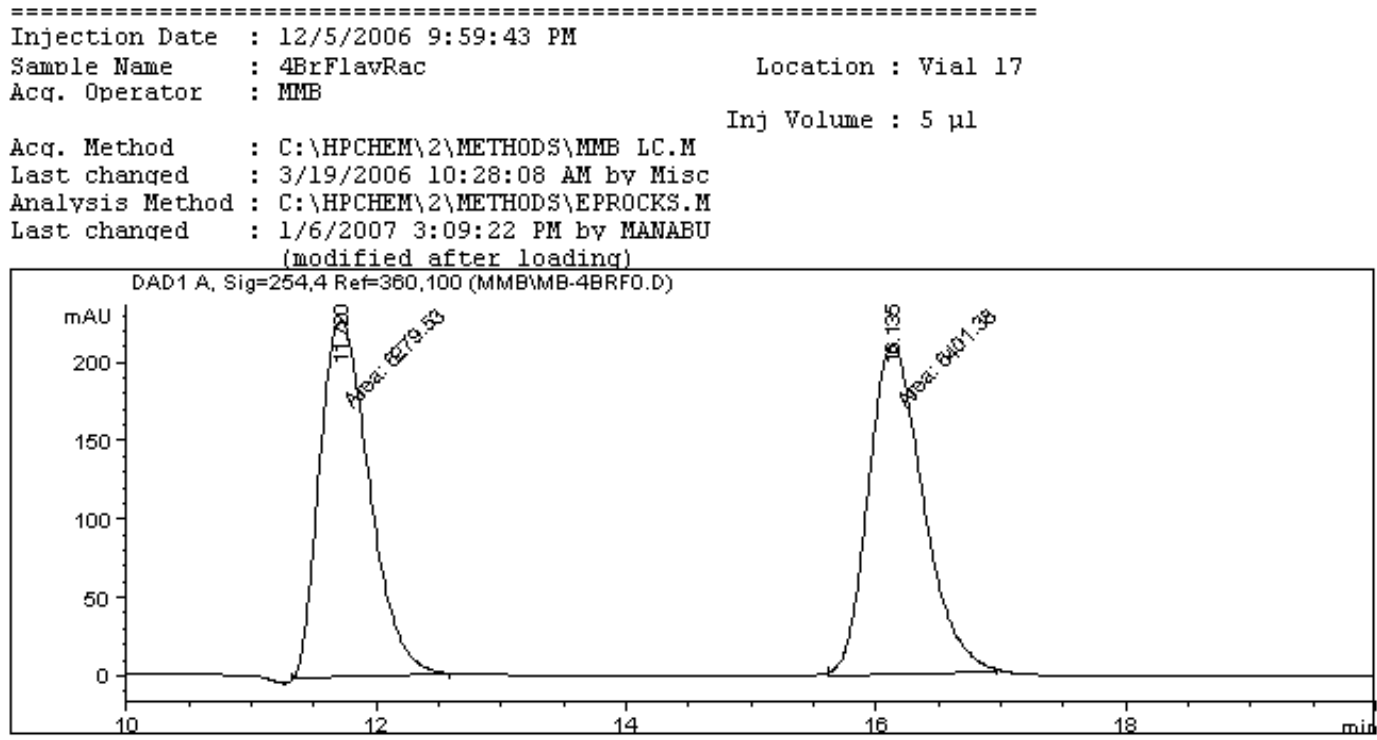

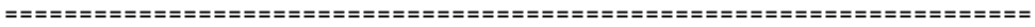

Area Percent Report

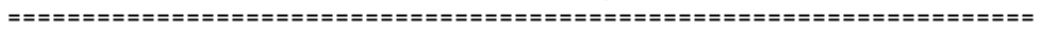

Sorted By : Sicnal

Multivlier : : 1.0000

Dilution : 1.0000

Use Multiplier \& Dilution Factor with ISTDs

Signal 1: DADl À, Sig $=254,4$ Ref $=360,100$

\begin{tabular}{|c|c|c|c|c|c|c|}
\hline $\begin{array}{c}\text { Peak } \\
\#\end{array}$ & $\begin{array}{c}\text { RetTime } \\
\text { 「min }\rceil\end{array}$ & Trpe & $\begin{array}{l}\text { Width } \\
\text { 「min }\end{array}$ & $\begin{array}{c}\text { Area } \\
\lceil\text { mAU* } \$\end{array}$ & $\begin{array}{l}\text { Height } \\
\text { 「mAU }\end{array}$ & $\underset{\substack{\text { Area } \\
\frac{\phi}{\delta}}}{ }$ \\
\hline 1 & 11.720 & $\mathrm{MM}$ & 0.4607 & 6279.52539 & 227.17545 & 195 \\
\hline 2 & 16.135 & $\mathrm{MM}$ & 0.5073 & 6401.38477 & 210.31682 & 4805 \\
\hline
\end{tabular}

Totals : $\quad 1.26809 \mathrm{e} 4 \quad 437.49226$

Results obtained with enhanced inteqrator!

柿 End of Report $\hbar \hbar \hbar$ 
Enantioenriched 2-(4-bromophenyl)chroman-4-one (8):

HPLC Conditions: 95:5 hexanes:isopropanol, $1.0 \mathrm{~mL} / \mathrm{min}$, OD-H Chiralcel column Data File C: \HPCHEM $\backslash \backslash \backslash$ DATA $\backslash M M B \backslash M \mathbb{E B} 307900 . \mathrm{D}$

$4 \mathrm{BrPh}$ flavanone

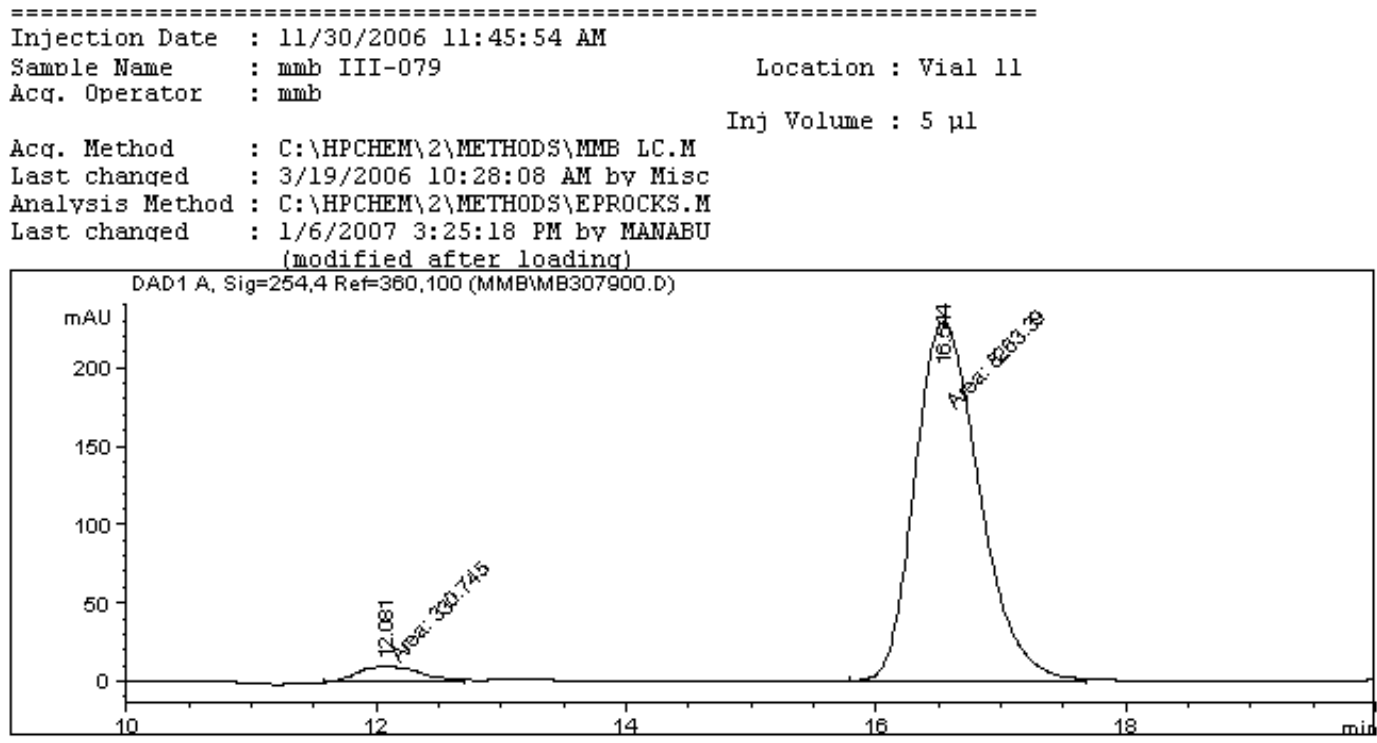

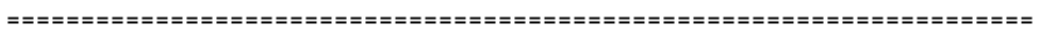

Area Percent Report

\begin{tabular}{|c|c|c|}
\hline Sorted By & : & Siqnal \\
\hline Multivlier & : & 1.0000 \\
\hline Dilution & : & 1.0000 \\
\hline
\end{tabular}

Use Multiplier \& Dilution Factor with IsTDs

Signal 1: DADl Aे, Sig=254, 4 Ref $=360,100$

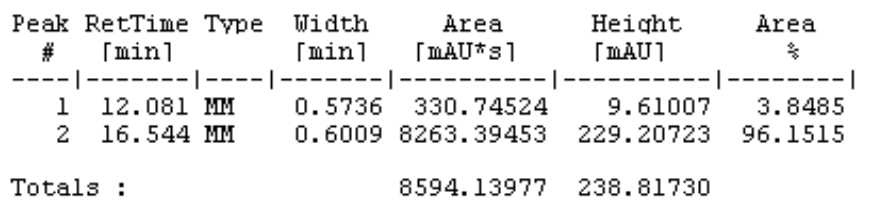

Results obtained with enhanced integrator!

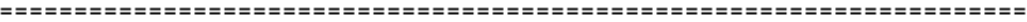

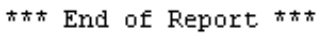




\section{Racemic 2-(2-naphthyl)chroman-4-one (9):}

HPLC Conditions: 90:10 hexanes:isopropanol, $1.0 \mathrm{~mL} / \mathrm{min}$, OD-H Chiralcel column Data File C: \HPCHEM $2 \nmid D A T A \backslash M M B \backslash M E N F L R A 1 . D$

NapFlav Rac

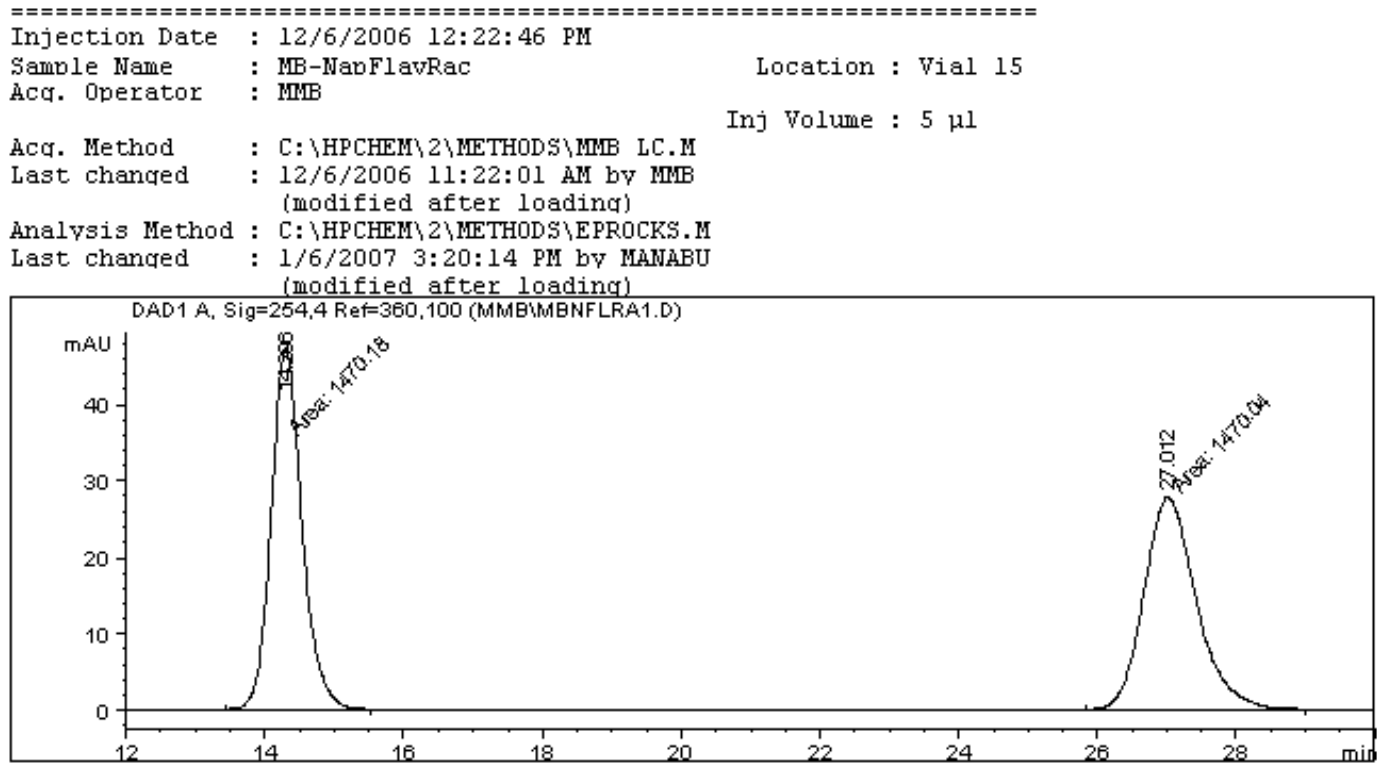

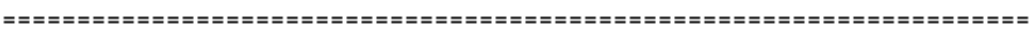
Area Percent Report

\begin{tabular}{|c|c|c|}
\hline $\begin{array}{l}\text { Sorted Bv } \\
\text { Multiplier }\end{array}$ & $\begin{array}{l}: \\
:\end{array}$ & $\begin{array}{l}\text { Sional } \\
1.0000\end{array}$ \\
\hline $\begin{array}{l}\text { Dilution } \\
\text { Use }\end{array}$ & : & 1.0000 \\
\hline
\end{tabular}

Use Multiplier \& Dilution Factor with ISTDs

Signal 1: DADl Aे, Sig=254, 4 Ref $=360,100$

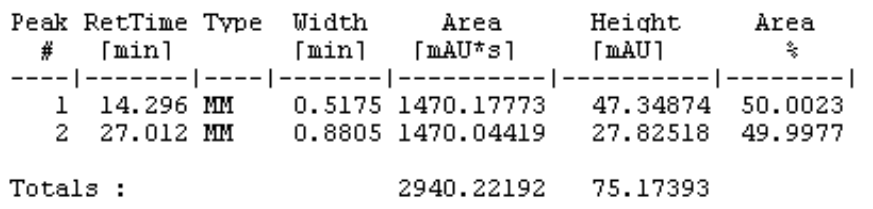

Results obtained with enhanced integrator!

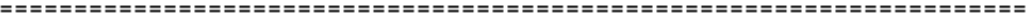
*** End of Report $* \pi$ 
Enantioenriched (R)-2-(2-naphthyl)chroman-4-one (9):

HPLC Conditions: 90:10 hexanes:isopropanol, $1.0 \mathrm{~mL} / \mathrm{min}$, OD-H Chiralcel column Data File C: $\backslash$ HPCHEM $\backslash \backslash \backslash$ DATA $\backslash M M B \backslash M E N A P 000 . D$

nap
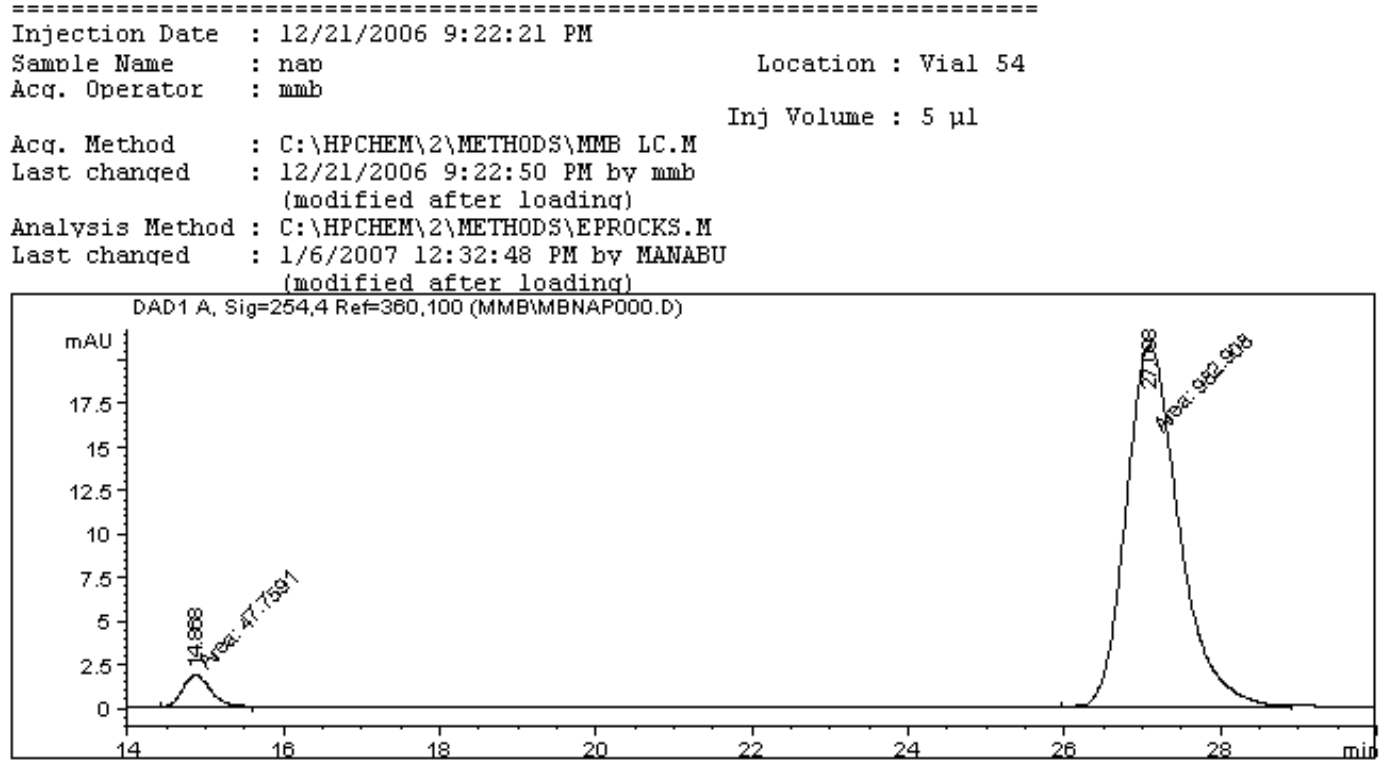

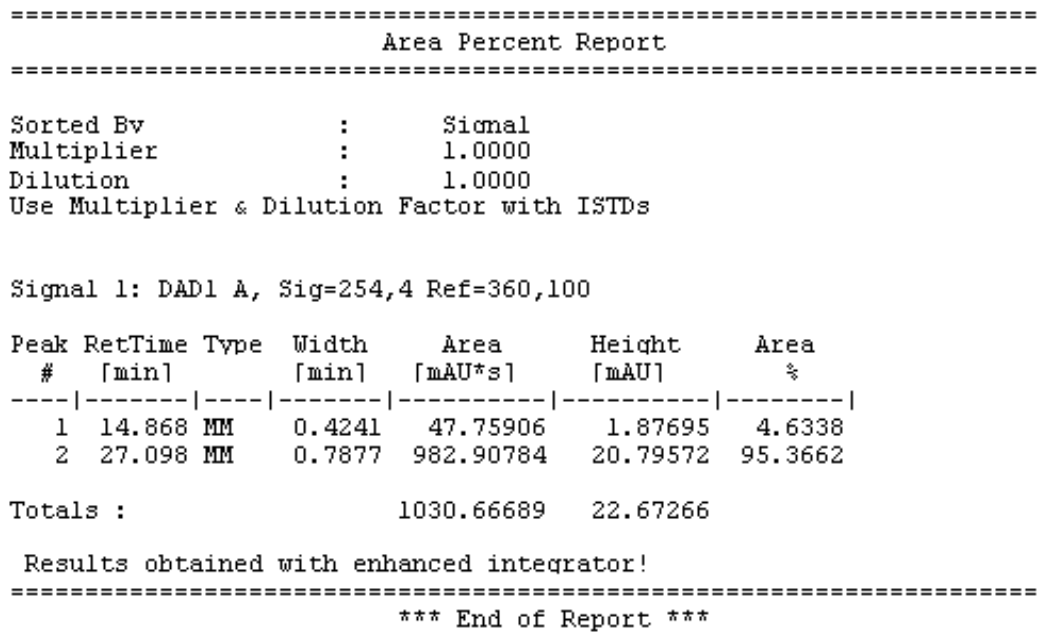

tof Report *t* 


\section{Racemic 2-p-tolylchroman-4-one (10):}

HPLC Conditions: 95:5 hexanes:isopropanol, $1.0 \mathrm{~mL} / \mathrm{min}$, OD-H Chiralcel column Data File C: $\backslash$ HPCHEM $2 \backslash D A T A M M M \backslash M B T F L R A 0 . D$

TolFlavRac
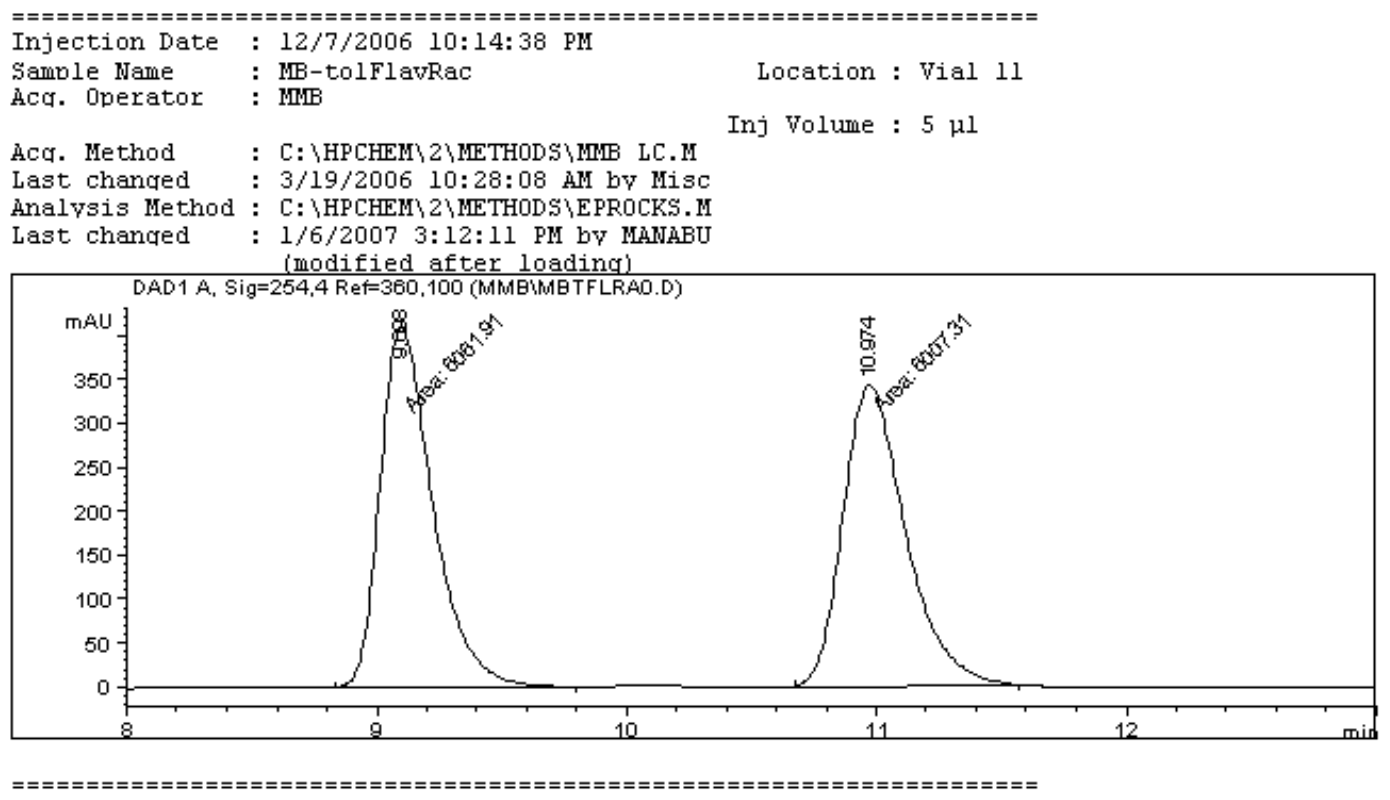

\section{Area Percent Report}

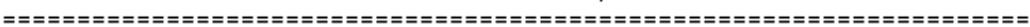

$\begin{array}{lll}\text { Sorted By } & : & \text { Sicmal } \\ \text { Multiolier } & : & 1.0000 \\ \text { Dilution } & : & 1.0000\end{array}$

Dilution 1.0000

Use Multiplier \& Dilution Factor with IsTDs

Signal 1: DADl A, Sig $=254,4$ Ref $=360,100$

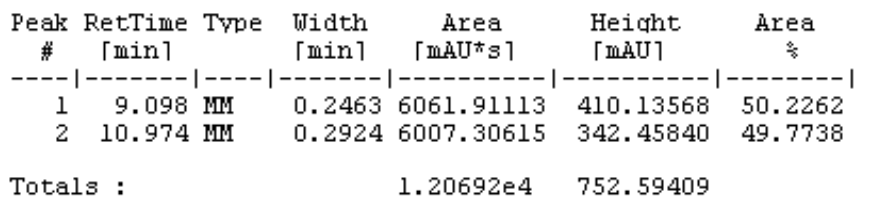

Results obtained with enhanced integrator!

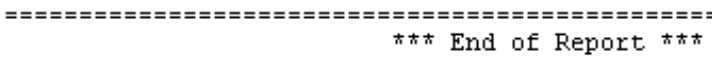


Enantioenriched (R)- 2-p-tolylchroman-4-one (10):

HPLC Conditions: 95:5 hexanes:isopropanol, $1.0 \mathrm{~mL} / \mathrm{min}$, OD-H Chiralcel column Data File C: \HPCHEM $2 \backslash D A T A \backslash M M \backslash M B 309330 . D$

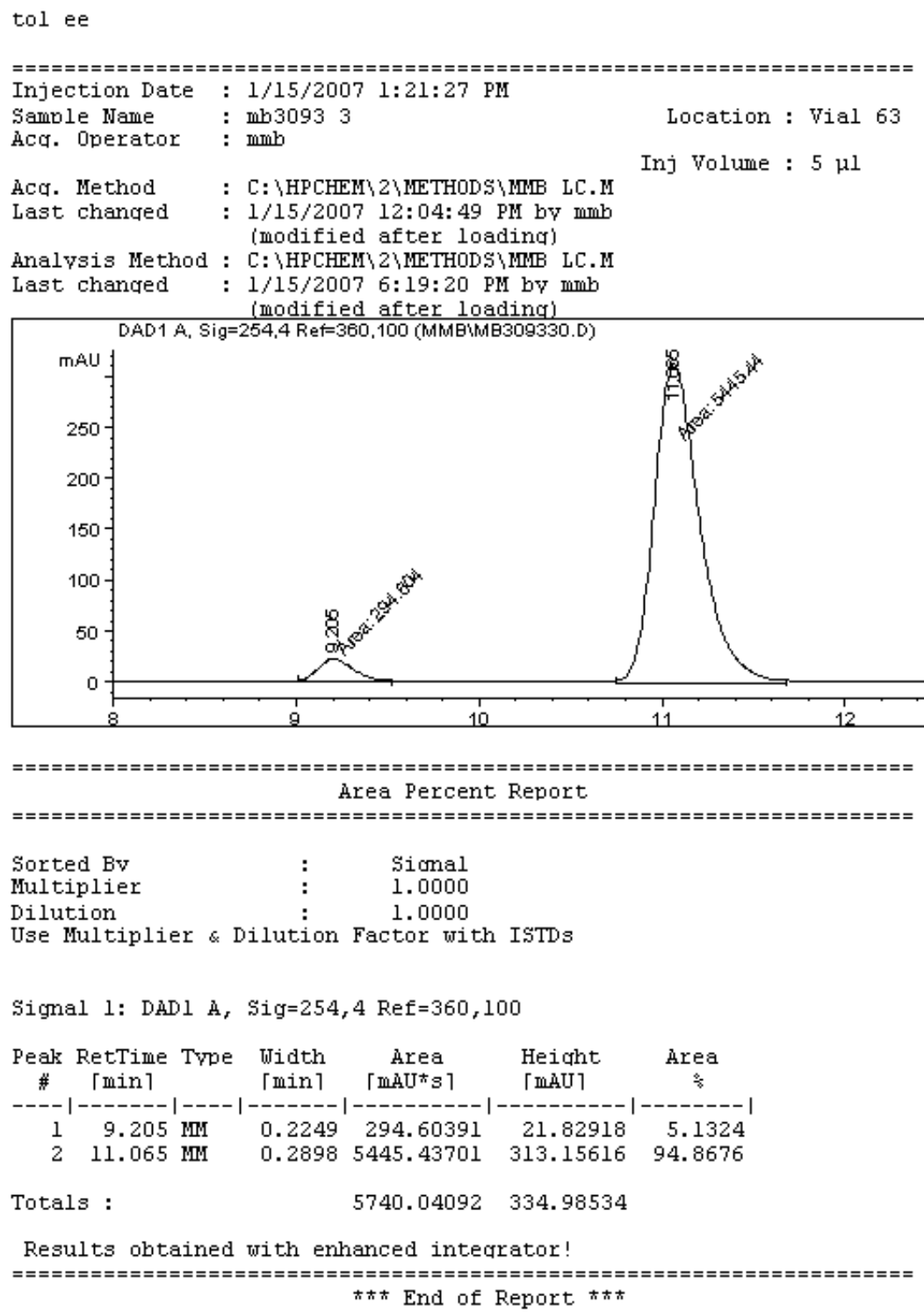

\#ネ End of Report 


\section{Racemic 2-(2-chlorophenyl)chroman-4-one (11):}

HPLC Conditions: 95:5 hexanes:isopropanol, $1.0 \mathrm{~mL} / \mathrm{min}$, OD-H Chiralcel column Data File C: \HPCHEM $2 \backslash D A T A \backslash M M \backslash M B 2 C L F 00 . D$

2 ClFlav Rac
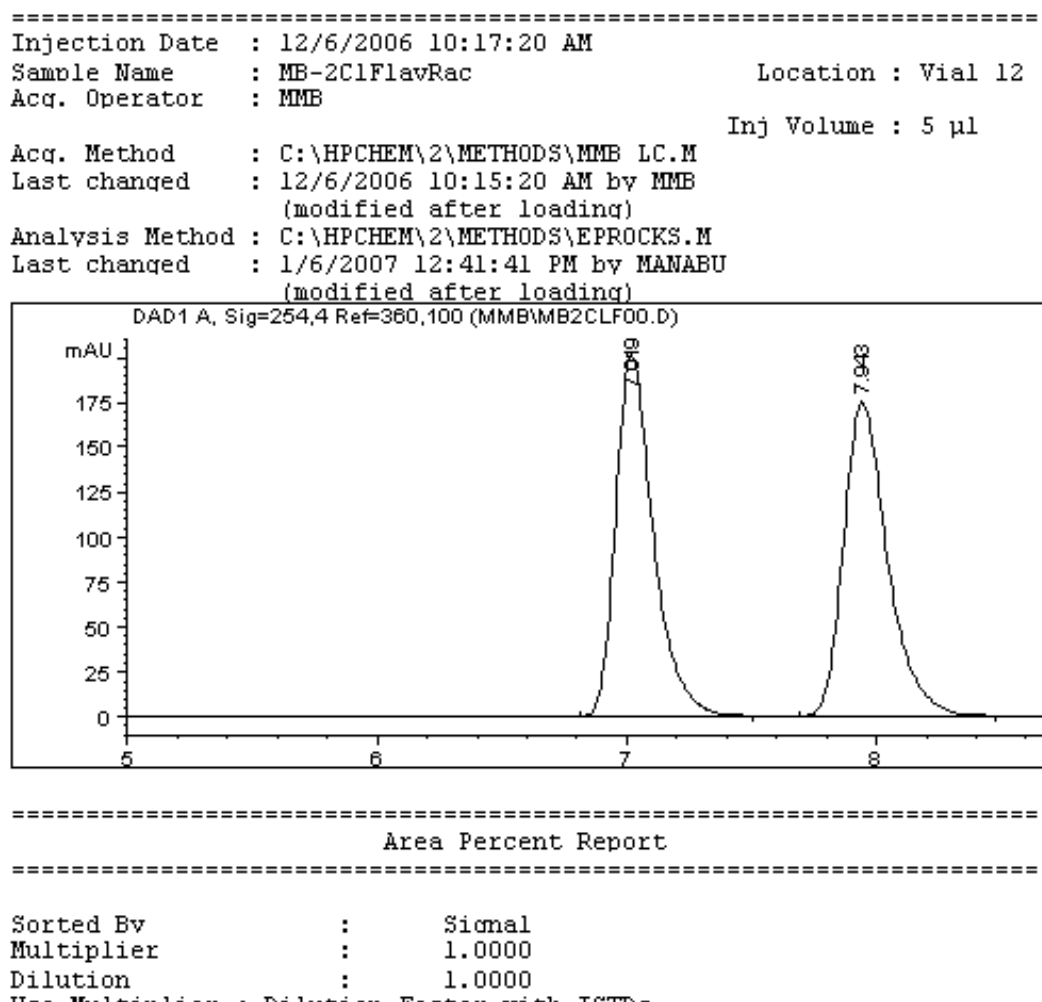

Dilution : 1.0000

Use Multiplier \& Dilution Factor with IsTDs

Signal 1: DADl À, Sig=254,4 Ref $=360,100$

\begin{tabular}{|c|c|c|c|c|c|c|}
\hline $\begin{array}{c}\text { Peak } \\
\#\end{array}$ & $\begin{array}{l}\text { RetTime } \\
\text { 「min } 1\end{array}$ & Type & $\begin{array}{l}\text { Width } \\
\text { 「min } 1\end{array}$ & $\begin{array}{c}\text { Area } \\
\left.\text { 「מAU } U^{*} s\right\rceil\end{array}$ & $\begin{array}{l}\text { Height } \\
\text { 「mid } 1\end{array}$ & $\underset{\substack{\text { Area } \\
\frac{5}{8}}}{ }$ \\
\hline & -1 & & & |---------- & ---------- & --------1 \\
\hline $\begin{array}{l}1 \\
2\end{array}$ & $\begin{array}{l}7.019 \\
7.943\end{array}$ & $\begin{array}{l}\mathrm{BB} \\
\mathrm{BB}\end{array}$ & $\begin{array}{l}0.1595 \\
0.1845\end{array}$ & $\begin{array}{l}2134.55908 \\
2137.02783\end{array}$ & $\begin{array}{l}201.62086 \\
174.95781\end{array}$ & $\begin{array}{l}49.9711 \\
50.0289\end{array}$ \\
\hline $\mathrm{t}$ & : & & & 4271.58691 & 376.57867 & \\
\hline
\end{tabular}

Results obtained with enhanced integrator! 
Enantioenriched (R)-2-(2-chlorophenyl)chroman-4-one (11):

HPLC Conditions: 95:5 hexanes:isopropanol, $1.0 \mathrm{~mL} / \mathrm{min}$, OD-H Chiralcel column Data File C: \HPCHEM $2 \backslash$ DATA $\backslash M \mathbb{B} \backslash M \mathbb{M B} 2 \mathrm{CL00.D}$

$2 \mathrm{Cl}$

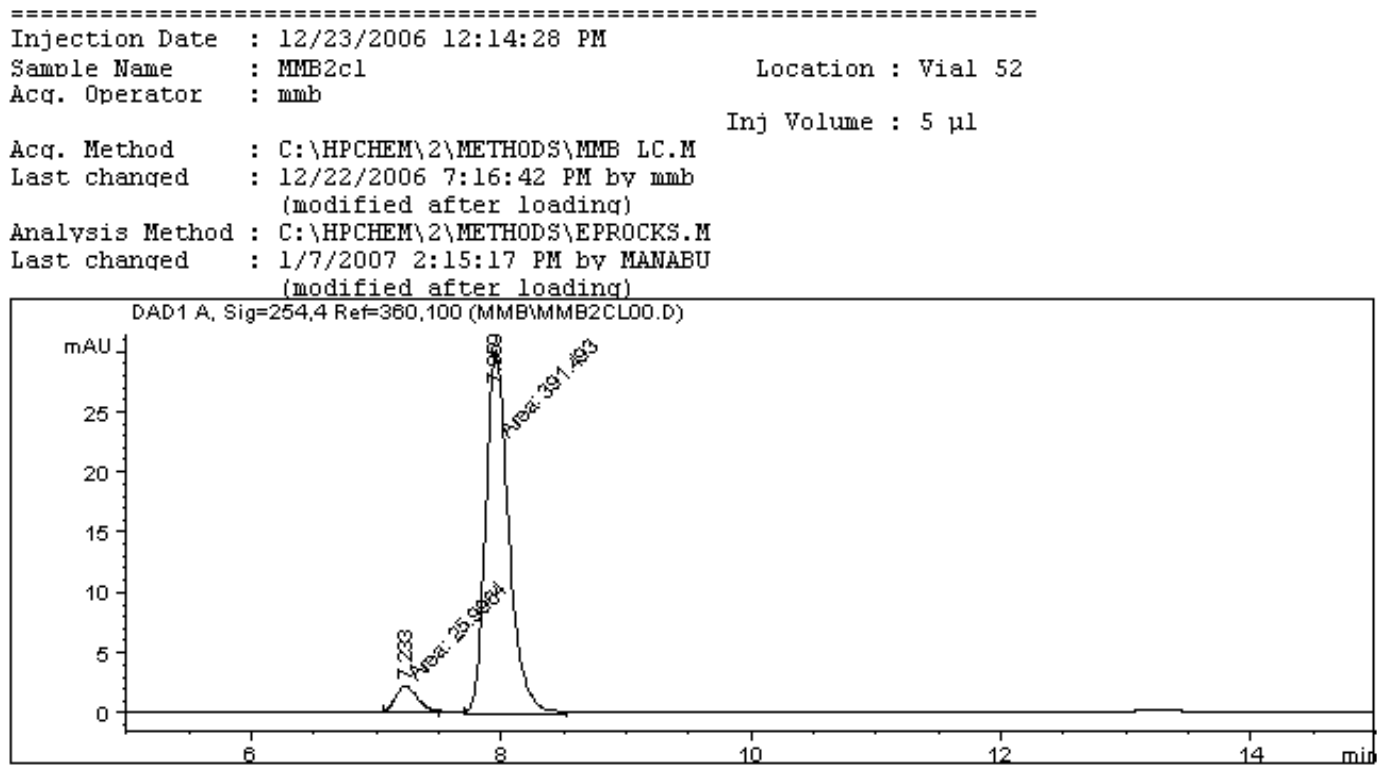

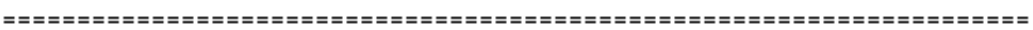

Area Percent Report

\begin{tabular}{|c|c|}
\hline Sorted Bv & : \\
\hline Multiplier & : \\
\hline
\end{tabular}

Dilution $\quad: \quad 1.0000$

Use Multiplier \& Dilution Factor with IsTDs

Signal 1: DADl A, Sig $=254,4$ Ref $=360,100$

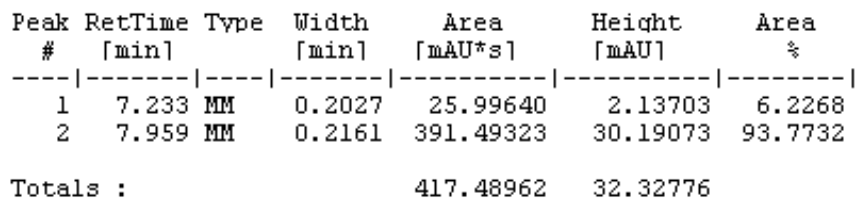

Results obtained with enhanced integrator!

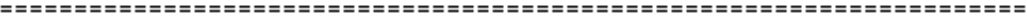

*** End of Report $\# *$ 
Racemic (2R,3S)-t-butyl 4-oxo-2-(4-methoxyphenyl)chroman-3-carboxylate (12): HPLC Conditions: 95:5 hexanes:isopropanol, $1.0 \mathrm{~mL} / \mathrm{min}$, OD-H Chiralcel column

Data File C: \HPCHEM $2 \backslash D A T A \backslash M M B \backslash 40 M E C Y R 0 . D$

40Me cy rac

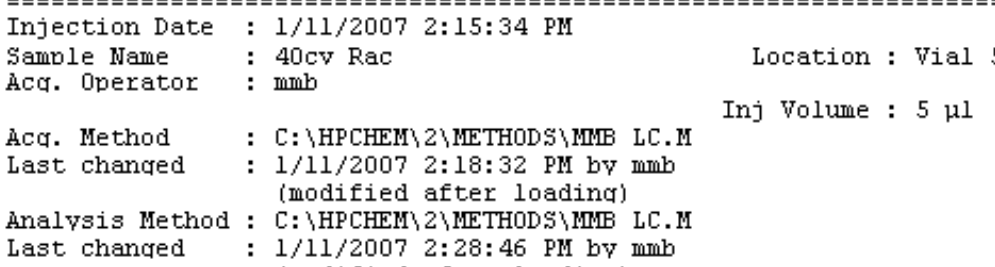

Inj Volume : $5 \mu l$

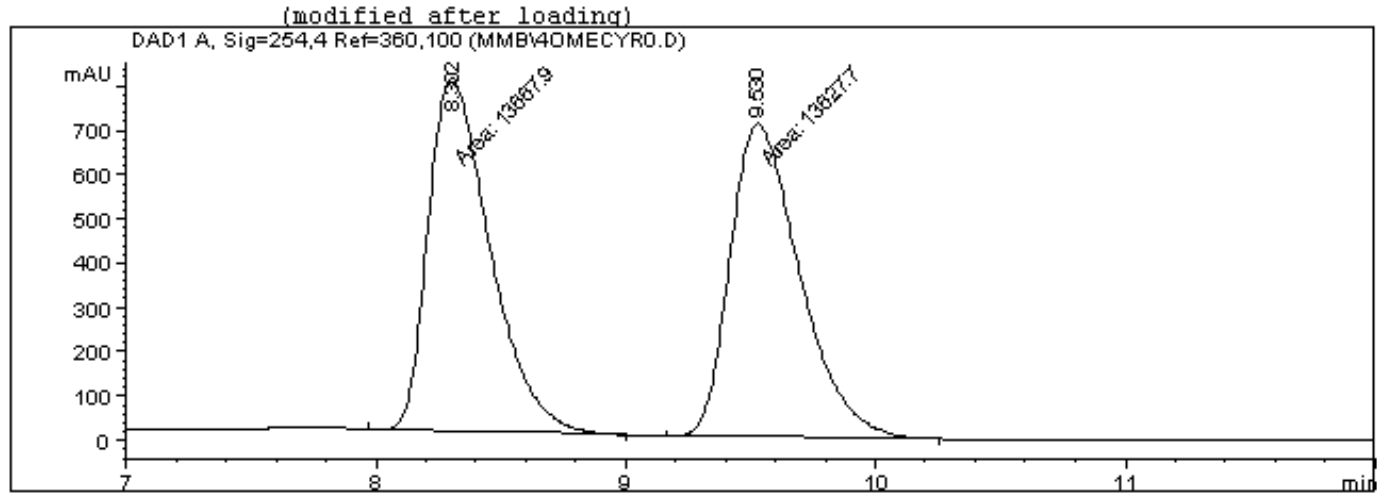

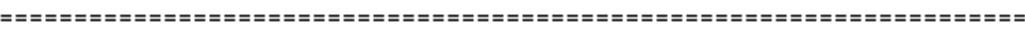

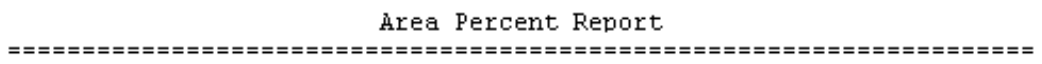

$\begin{array}{lll}\text { Sorted Bv } & : & \text { Sional } \\ \text { Multiplier } & : & 1.0000\end{array}$

Dilution $\quad 1.0000$

Use Multiplier \& Dilution Factor with IsTDs

Signal 1: DADl A, Sig=254,4 Ref $=360,100$

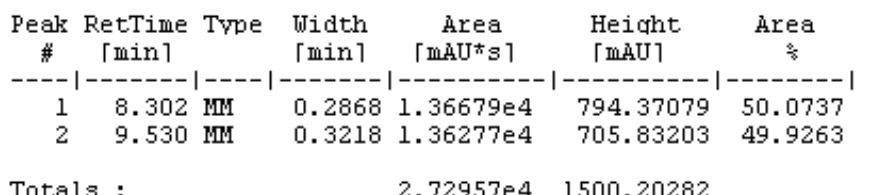

Totals : $\quad 2.72957 \mathrm{e} 4 \quad 1500.20282$

Results obtained with enhanced integrator!

*木 End of Report 


\section{Enantioenriched (2R,3S)-t-butyl 4-oxo-2-(4-methoxyphenyl)chroman-3-carboxylate} (12):

HPLC Conditions: 95:5 hexanes:isopropanol, $1.0 \mathrm{~mL} / \mathrm{min}$, OD-H Chiralcel column

Data File C: \HPCHEM $2 \backslash D A T A \backslash M \mathbb{M B} \backslash \mathbb{M B} 3032 . D$

4 ' $\mathrm{MePh}$
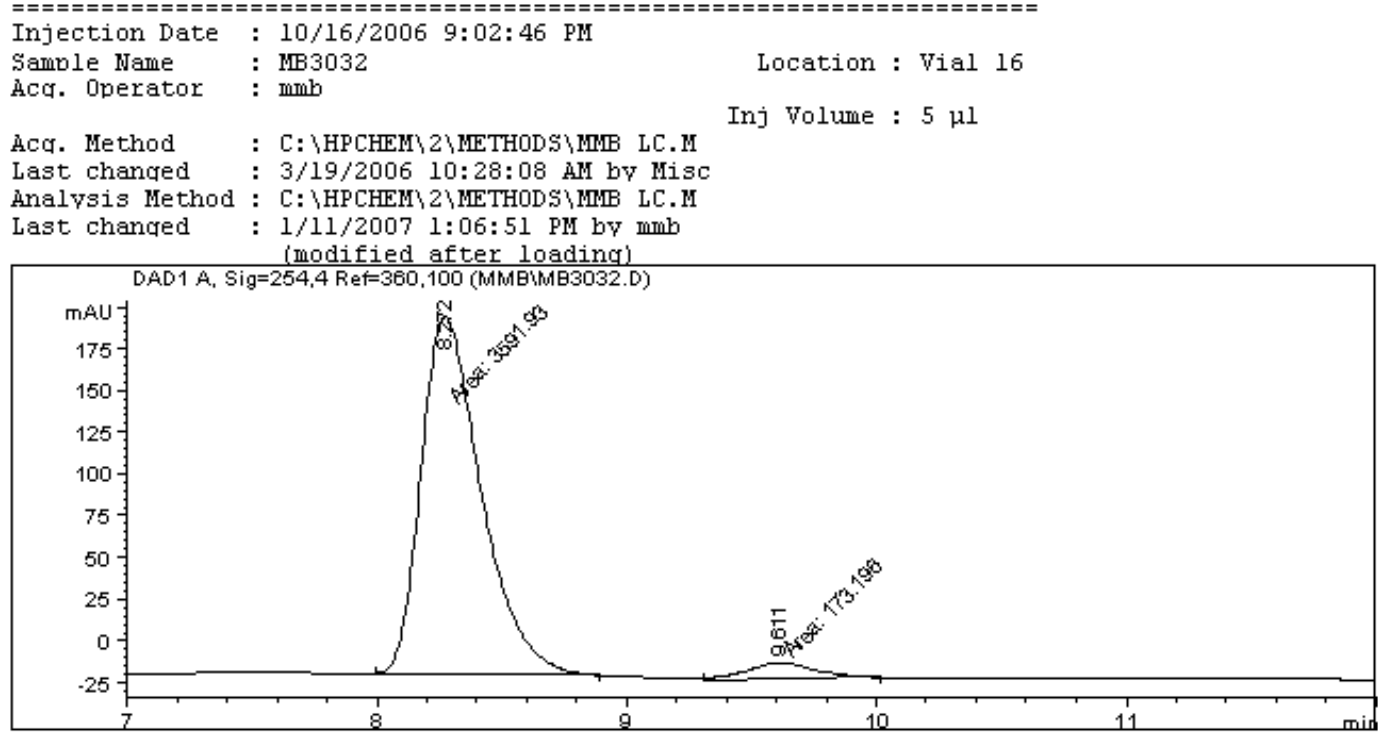

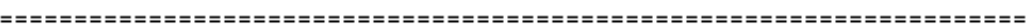
Area Percent Report

\begin{tabular}{|c|c|c|}
\hline Sorted By & : & Siqnal \\
\hline Multiolier & : & 1.0000 \\
\hline Dilution & : & 1.0000 \\
\hline
\end{tabular}

Use Multiplier \& Dilution Factor with IsTDs

Signal 1: DADl Aे, Sig=254, 4 Ref $=360,100$

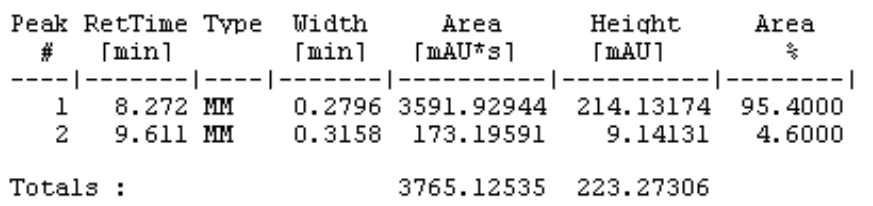

Results obtained with enhanced integrator!

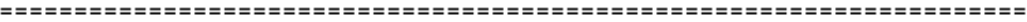
ネネ⿱ End of Report *ネ 


\section{Racemic 2-(4-methoxyphenyl)chroman-4-one:}

HPLC Conditions: 95:5 hexanes:isopropanol, $1.0 \mathrm{~mL} / \mathrm{min}$, OD-H Chiralcel column

Data File C: \HPCHEM $2 \backslash$ DATA $\backslash M M B \backslash 40 M E F L R O . D \quad$ Sample Name: $40 f l a v$ Rac

40Me flav rac
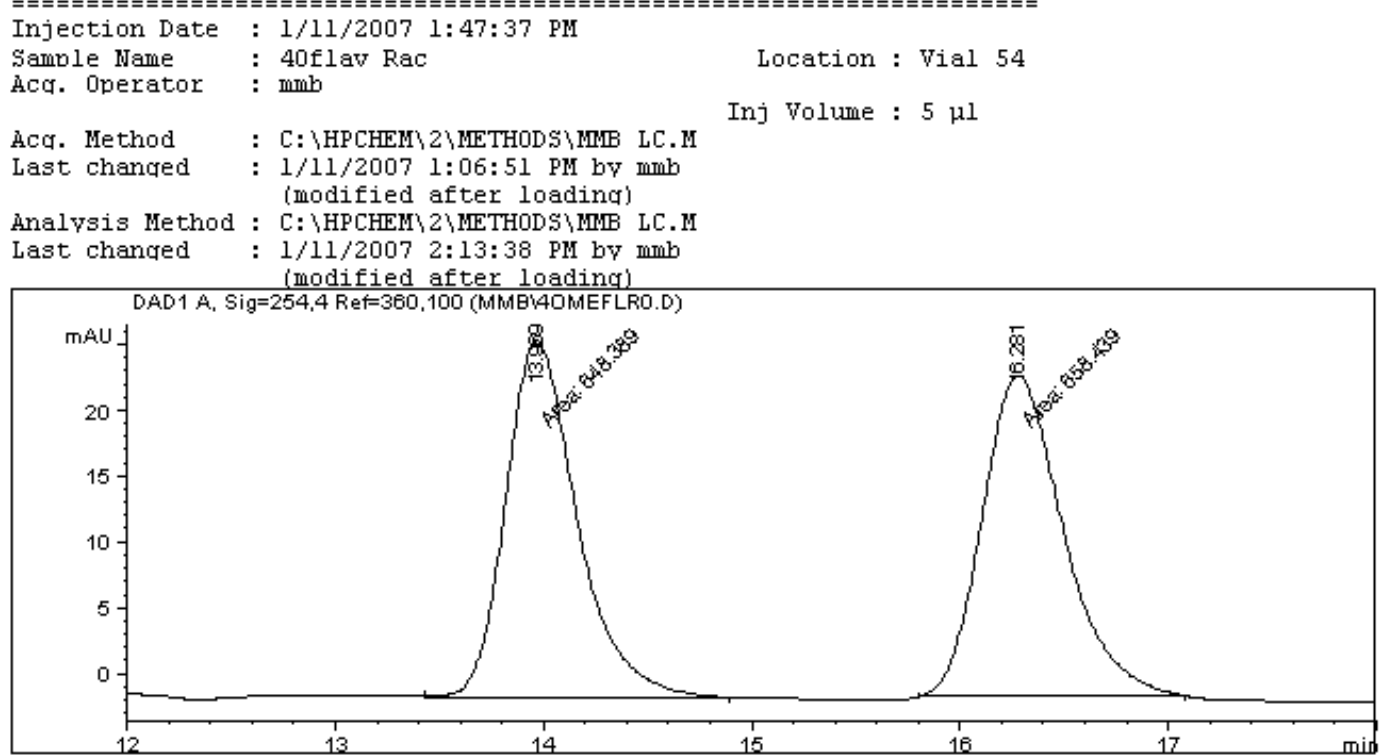

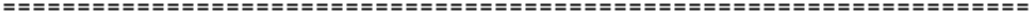

Area Percent Report

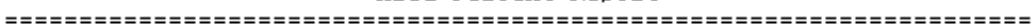

$\begin{array}{lll}\text { Sorted Bv } & : & \text { Sicmal } \\ \text { Multiplier } & : & 1.0000 \\ \text { Dilution } & : & 1.0000\end{array}$

Dilution $\quad: \quad 1.0000$

Use Multiplier \& Dilution Factor with ISTDs

Signal 1: DADl A, Sig $=254,4$ Ref $=360,100$

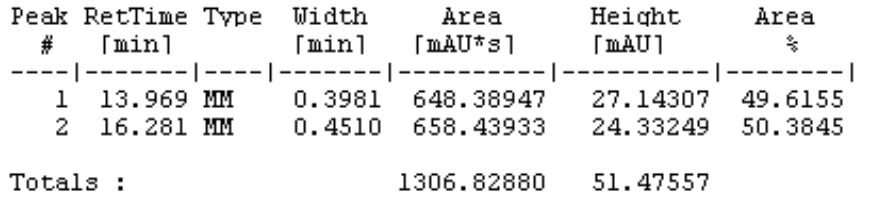

Results obtained with enhanced integrator! 


\section{Enantioenriched $(R)$-2-(4-methoxyphenyl)chroman-4-one:}

HPLC Conditions: 95:5 hexanes:isopropanol, $1.0 \mathrm{~mL} / \mathrm{min}$, OD-H Chiralcel column

Data File C: \HPCHEM\2\DATẢMMB $\backslash M M B 31280 . D$

40MeMgBr2
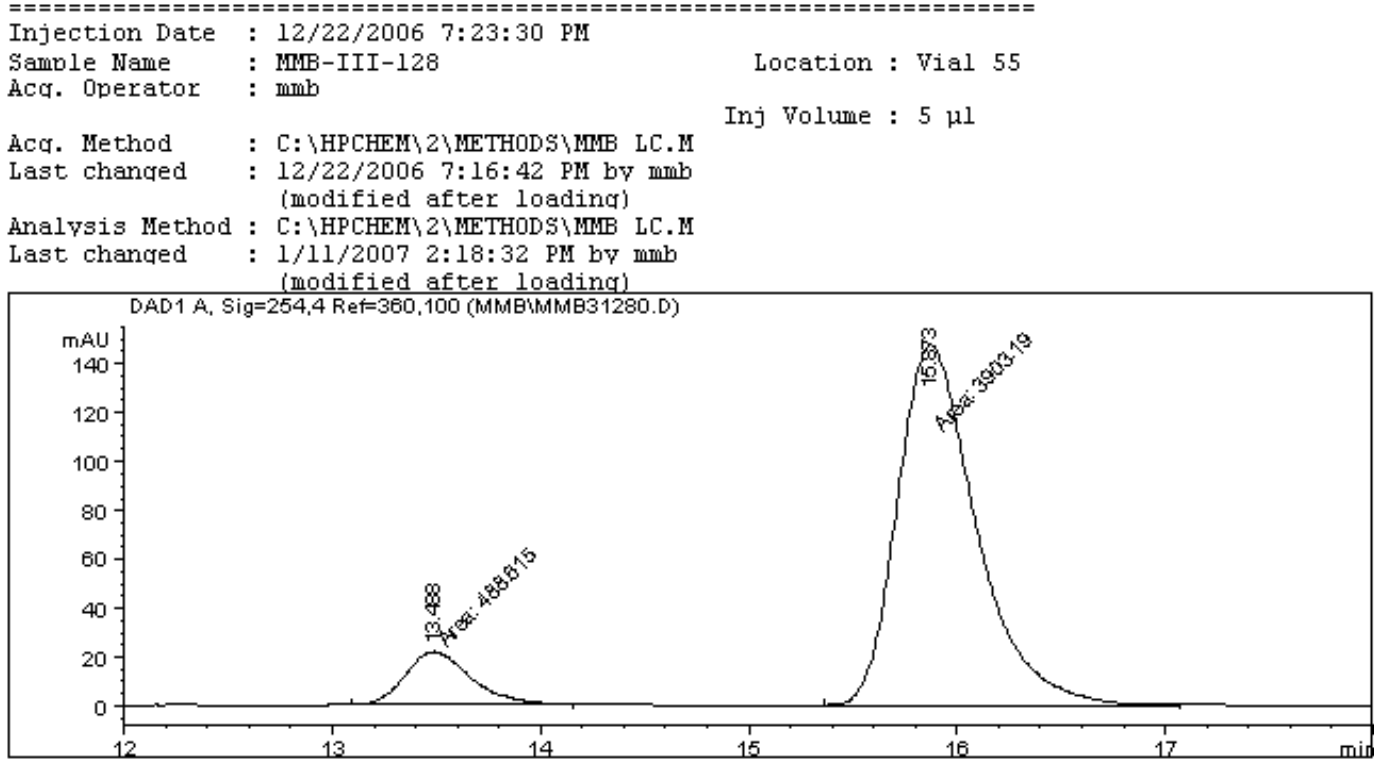

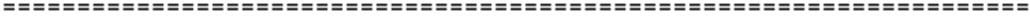

Area Percent Report

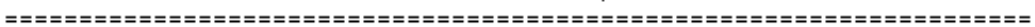

$\begin{array}{lll}\text { Sorted Bv } & : & \text { Sional } \\ \text { Multiplier } & : & 1.0000 \\ \text { Dilution } & : & 1.0000\end{array}$

Dilution $\quad: \quad 1.0000$

Use Multiplier \& Dilution Factor with ISTDs

Signal 1: DADl Aे, Sig $=254,4$ Ref $=360,100$

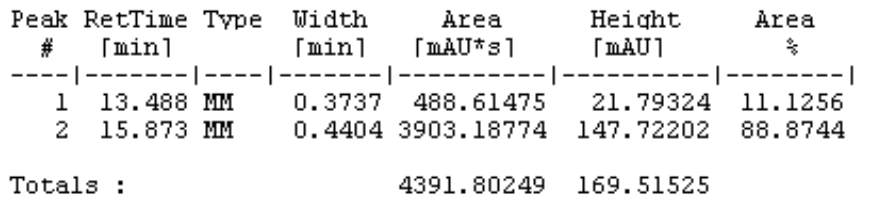

Results obtained with enhanced integrator! 


\section{Racemic 7-methoxy-2-phenylchroman-4-one (13):}

HPLC Conditions: 95:5 hexanes:isopropanol, $1.0 \mathrm{~mL} / \mathrm{min}$, OD-H Chiralcel column

Data File C: \HPCHEM\2\DATA \MB \MB70MER0.D Sample Name: MB-70MeFlavRac

70MeFlavRac
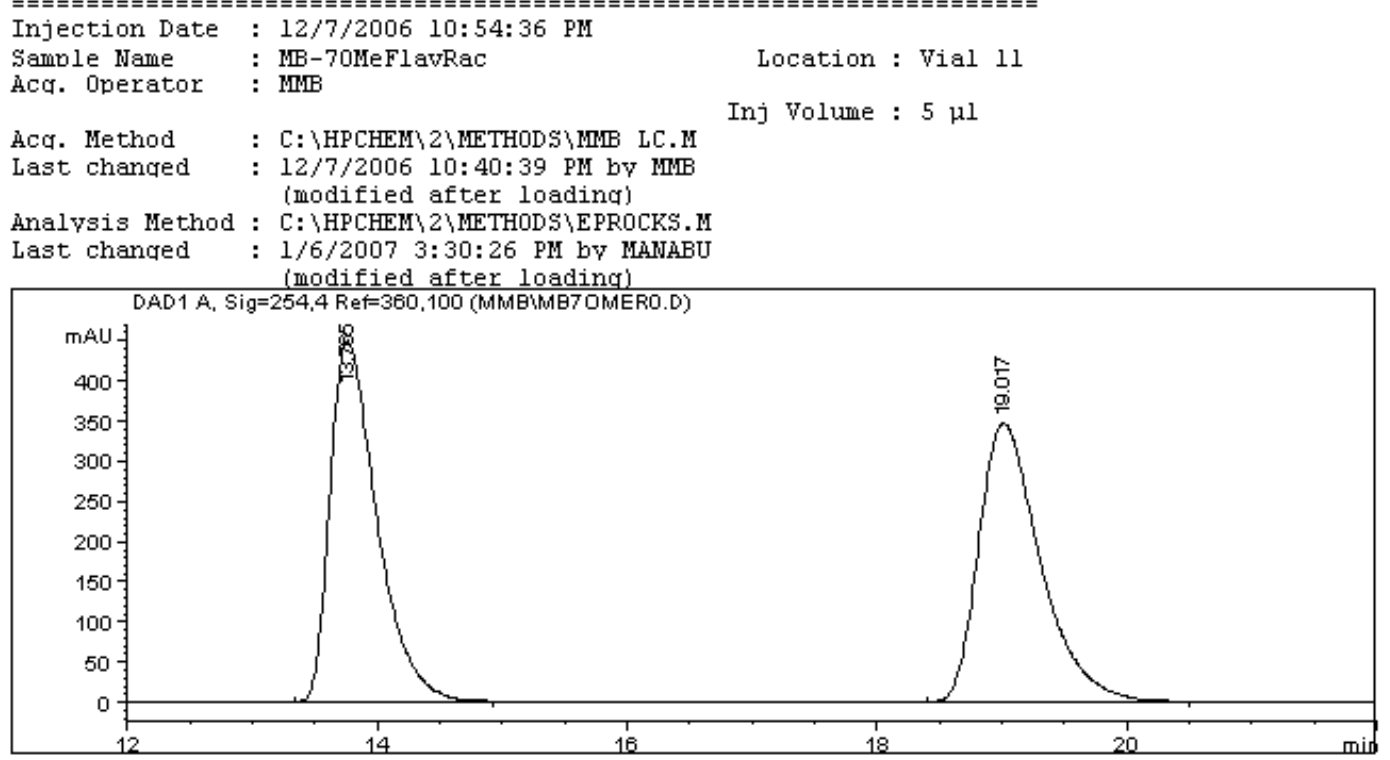

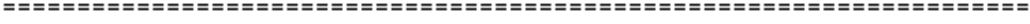

Area Percent Report

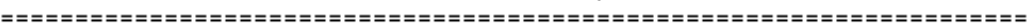

$\begin{array}{lll}\text { Sorted Bv } & : & \text { Sional } \\ \text { Multiplier } & : & 1.0000 \\ \text { Dilution } & : & 1.0000\end{array}$

Dilution $\quad: \quad 1.0000$

Use Multiplier \& Dilution Factor with ISTDs

Signal 1: DADl Aे, Sig=254, 4 Ref $=360,100$

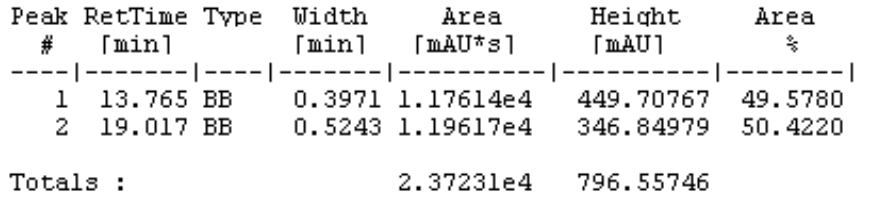

Results obtained with enhanced integrator!

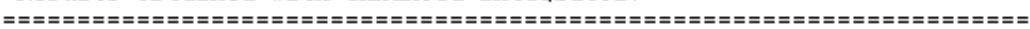

ホネ End of Report 
Enantioenriched (R)-7-methoxy-2-phenylchroman-4-one (13): HPLC Conditions: 95:5 hexanes:isopropanol, $1.0 \mathrm{~mL} / \mathrm{min}$, OD-H Chiralcel column Data File C: $\backslash$ HPCHEM $\backslash \backslash \backslash$ DATA $\backslash M M \mathbb{B} \backslash M \mathbb{M B} 3-8300 . \mathrm{D}$

$70 \mathrm{Me}$
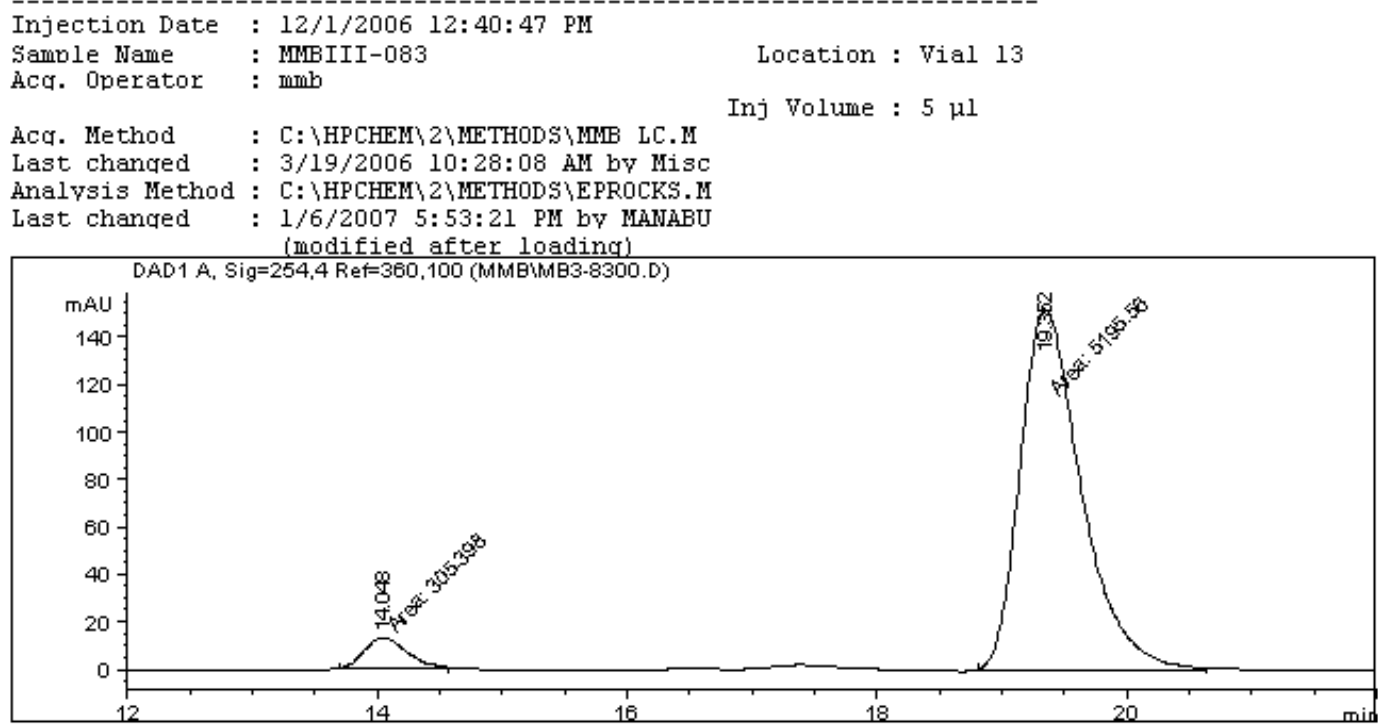

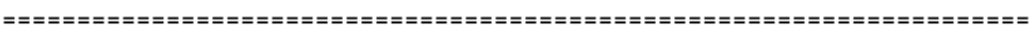

Area Percent Report

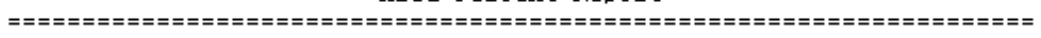

Sorted By $\quad$ : $\quad$ Siqnal

Multivlier $\quad: \quad 1.0000$

Dilution

Use Multiplier \& Dilution Factor with ISTDs

Signal 1: DADD À, Sig $=254,4$ Ref $=360,100$

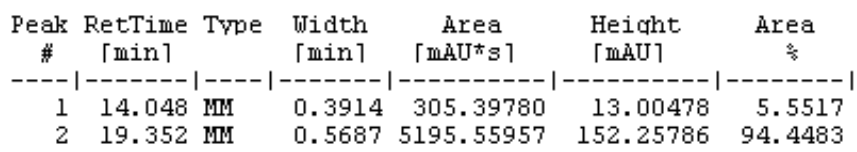

Totals :

$5500.95737 \quad 165.26264$

Results obtained with enhanced inteqrator!

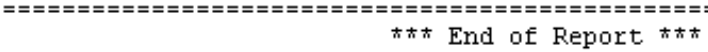




\section{Racemic 7-methoxy-2-phenylchroman-4-one (14):}

HPLC Conditions: 95:5 hexanes:isopropanol, $1.0 \mathrm{~mL} / \mathrm{min}$, OD-H Chiralcel column Data File C: \HPCHEM $2 \backslash$ DATẢ $\backslash M M \backslash 7 M E F L R A l . D$

7 Me flav rac

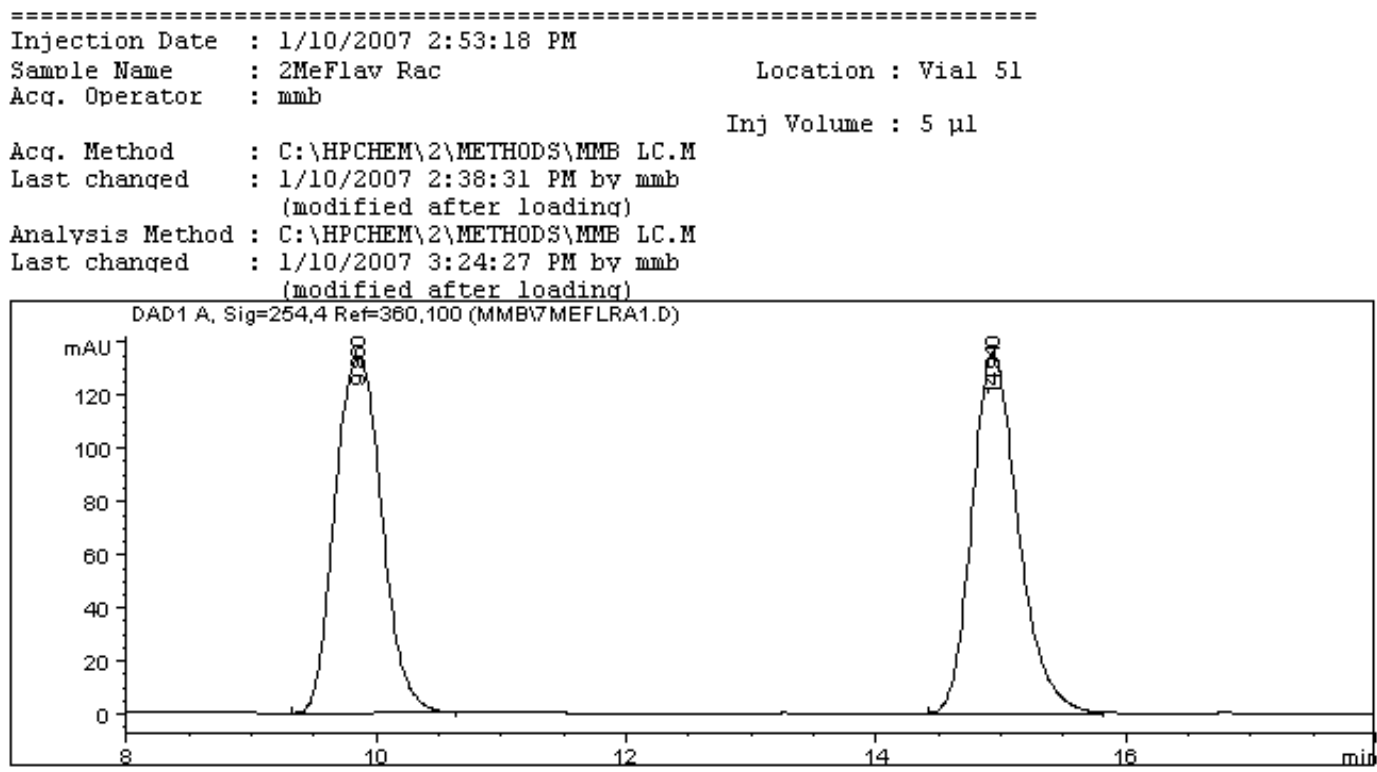

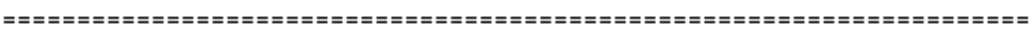

Area Percent Report

\begin{tabular}{|c|c|c|}
\hline $\begin{array}{l}\text { Sorted Bv } \\
\text { Multiplier }\end{array}$ & $\begin{array}{l}: \\
\vdots\end{array}$ & $\begin{array}{l}\text { Sional } \\
1.0000\end{array}$ \\
\hline $\begin{array}{l}\text { Dilution } \\
\text { Use Multipl }\end{array}$ & : & $\begin{array}{l}1.0000 \\
t \operatorname{tor}\end{array}$ \\
\hline
\end{tabular}

Use Multiplier \& Dilution Factor with ISTDs

Signal 1: DADl Aे, Sig=254, 4 Ref $=360,100$

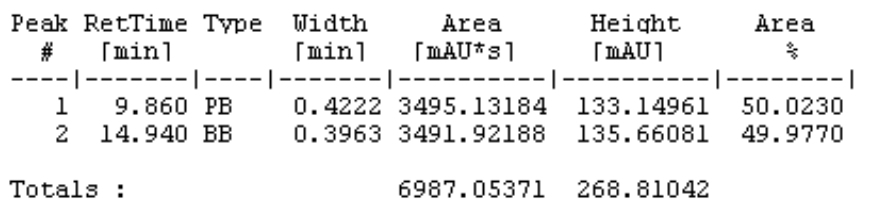

Results obtained with enhanced integrator!

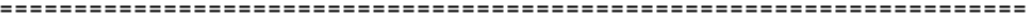

ネネー End of Report *ネ 


\section{Enantioenriched $(\boldsymbol{R})$-7-methyl-2-phenylchroman-4-one (14):}

HPLC Conditions: 95:5 hexanes:isopropanol, $1.0 \mathrm{~mL} / \mathrm{min}$, OD-H Chiralcel column Data File C: \HPCHEM $2 \backslash D A T A \backslash M M \backslash M B 313430 . D$

7 Me ee

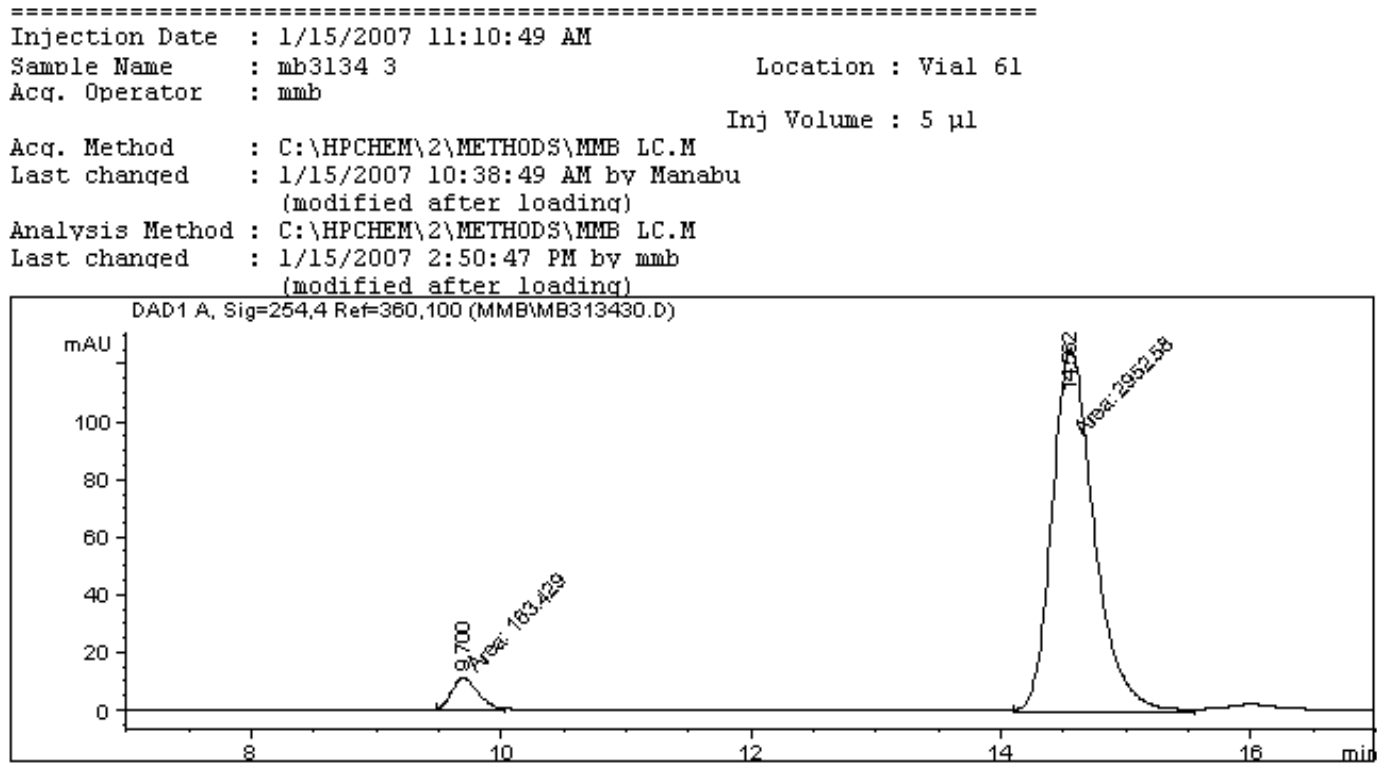

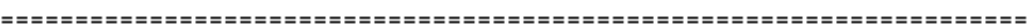

Area Percent Report

\begin{tabular}{|c|c|}
\hline Sorted Bv & : \\
\hline Multiplier & : \\
\hline
\end{tabular}

Dilution : 1.0000

Use Multiplier \& Dilution Factor with IsTDs

Signal 1: DADl Aे, Sig=254, 4 Ref $=360,100$

\begin{tabular}{|c|c|c|c|c|c|c|}
\hline $\begin{array}{c}\text { Peak } \\
\#\end{array}$ & $\begin{array}{c}\text { RetTime } \\
\text { 「min } 1\end{array}$ & Trpe & $\begin{array}{l}\text { Width } \\
\text { 「min }\rceil\end{array}$ & $\begin{array}{c}\text { Area } \\
\text { 「mAUt's } 1\end{array}$ & $\begin{array}{l}\text { Height } \\
\text { 「mAU }\rceil\end{array}$ & Area \\
\hline 2 & $\begin{array}{r}9.700 \\
14.562\end{array}$ & MM & $\begin{array}{l}0.2465 \\
0.3903\end{array}$ & $\begin{array}{r}163.42935 \\
2952.58057\end{array}$ & $\begin{array}{r}11.04830 \\
126.08385\end{array}$ & $\begin{array}{r}5.2448 \\
94.7552\end{array}$ \\
\hline
\end{tabular}

Totals : $\quad 3116.00992 \quad 137.13216$

Results obtained with enhanced integrator!

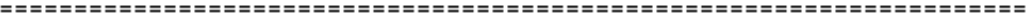

\#ネ* End of Report ה木 


\section{Racemic 6,7-benzo-2-phenylchroman-4-one (15):}

HPLC Conditions: 90:10 hexanes:isopropanol, $1.0 \mathrm{~mL} / \mathrm{min}$, OD-H Chiralcel column

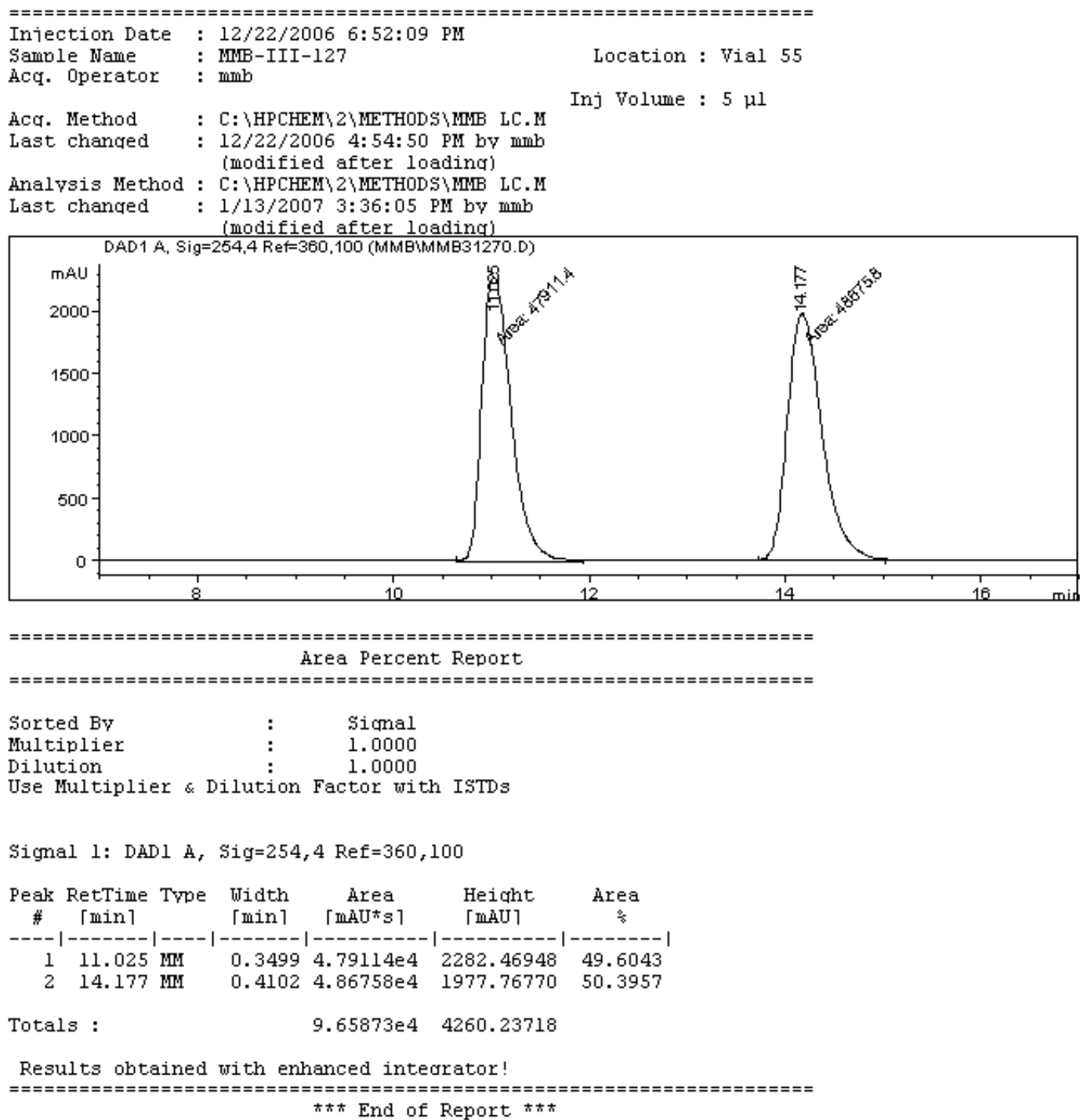


Enantioenriched $(\boldsymbol{R})$-6,7-benzo-2-phenylchroman-4-one (15):

HPLC Conditions: 90:10 hexanes:isopropanol, $1.0 \mathrm{~mL} / \mathrm{min}$, OD-H Chiralcel column

Data File C: \HPCHEM $2 \backslash D A T A \backslash M M B \backslash M B 313020 . D$

2,3 nap ee dilute

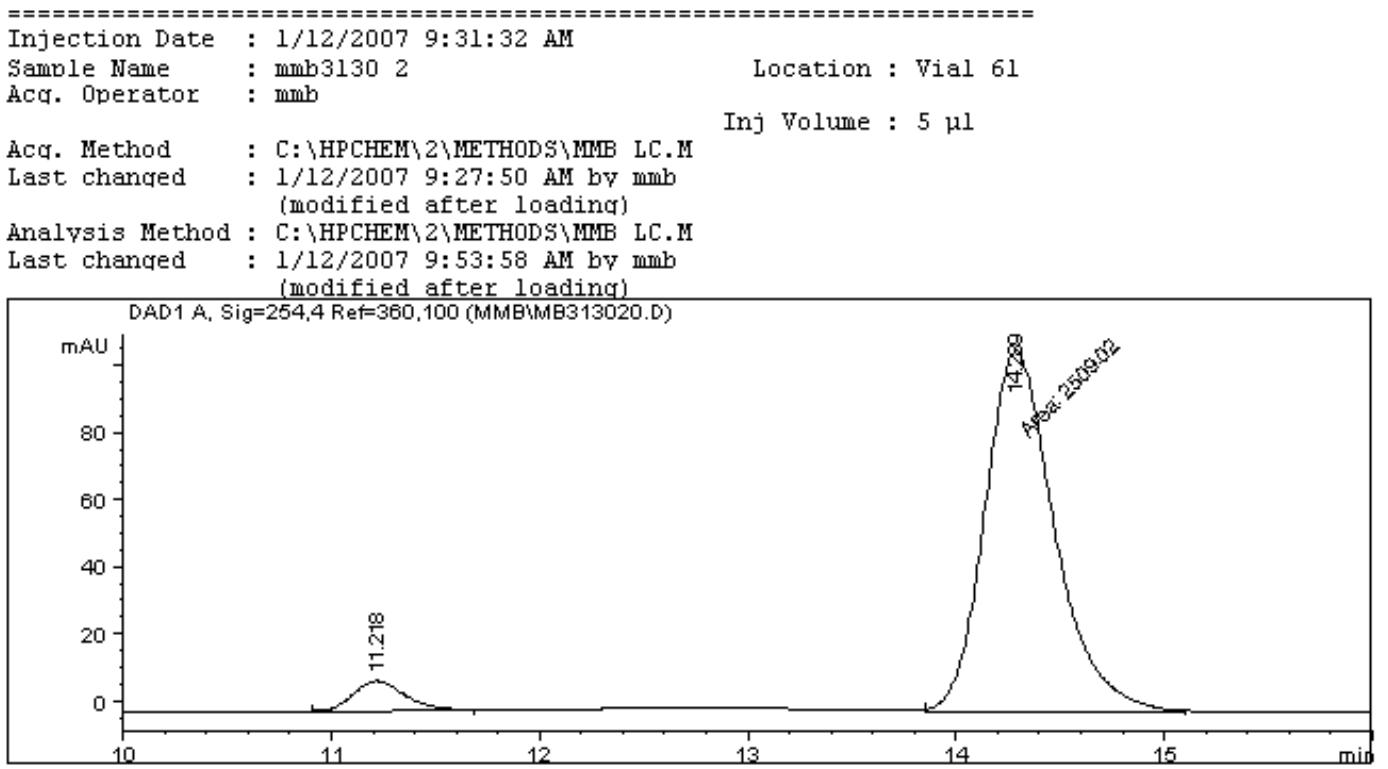

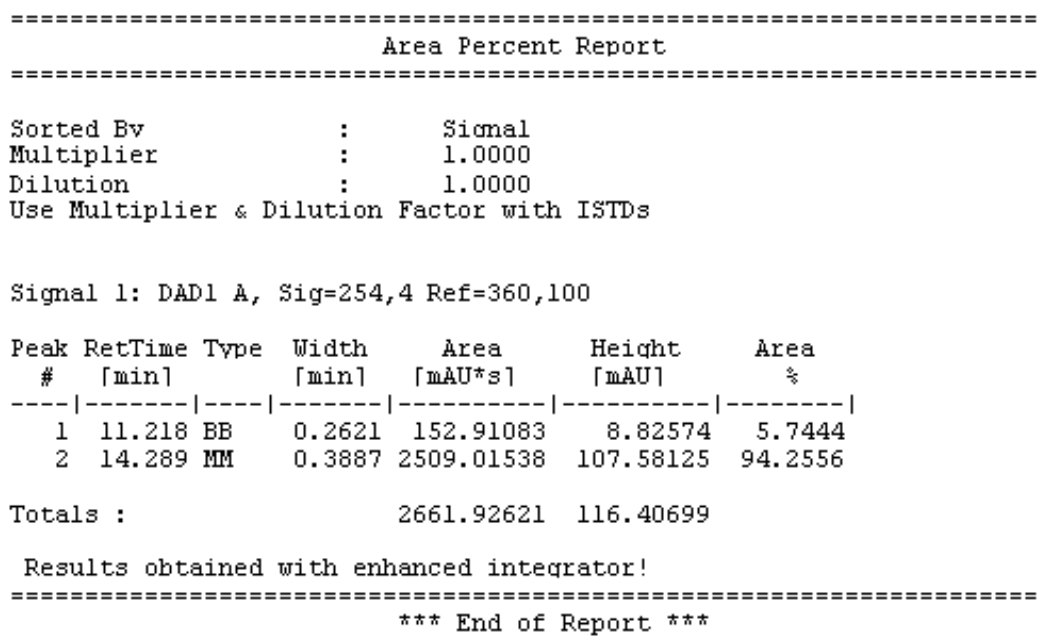

End of Report $* * \pi$ 


\section{Racemic 2-(cyclohexyl)chroman-4-one (16):}

HPLC Conditions: 99:1 hexanes:isopropanol, $0.3 \mathrm{~mL} / \mathrm{min}$, OD-H Chiralcel column Data File C: \HPCHEM\2\DATA \MM \CYHEXRAO.D

cyclohexylrac flav

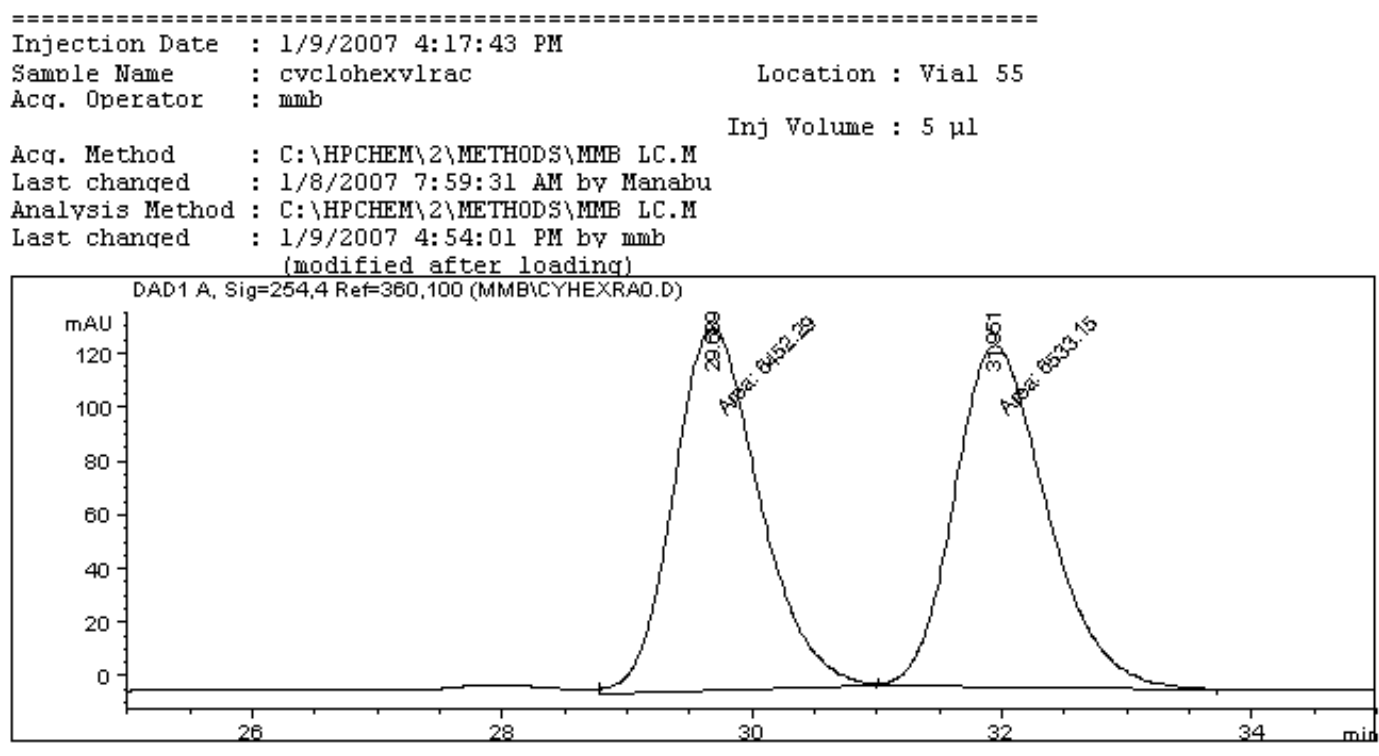

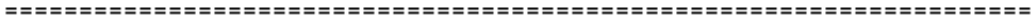

hrea Percent Report

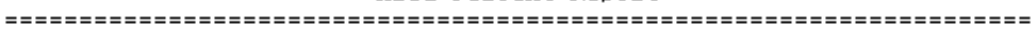

$\begin{array}{lll}\text { Sorted By } & : & \text { Siqnal } \\ \text { Multiblier } & \vdots & 1.0000 \\ \text { Dilution } & : & 1.0000\end{array}$

Use Multiplier \& Dilution Factor with ISTDs

Signal 1: DADl A, Sig=254, 4 Ref $=360,100$

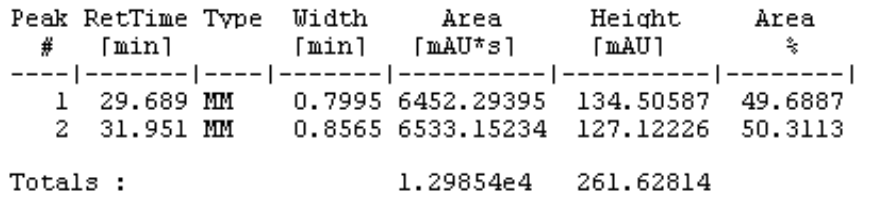

Results obtained with enhanced inteqrator!

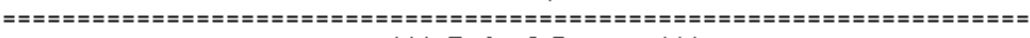

ネネネ End of Report 
Enantioenriched $(\boldsymbol{R})$-2-(cyclohexyl)chroman-4-one (16):

HPLC Conditions: 99:1 hexanes:isopropanol, $0.3 \mathrm{~mL} / \mathrm{min}$, OD-H Chiralcel column Data File C: $\backslash$ HPCHEM $\backslash \backslash$ \ATA $\backslash M M B \backslash C Y H E X E E O . D$

cyclohexylee flav

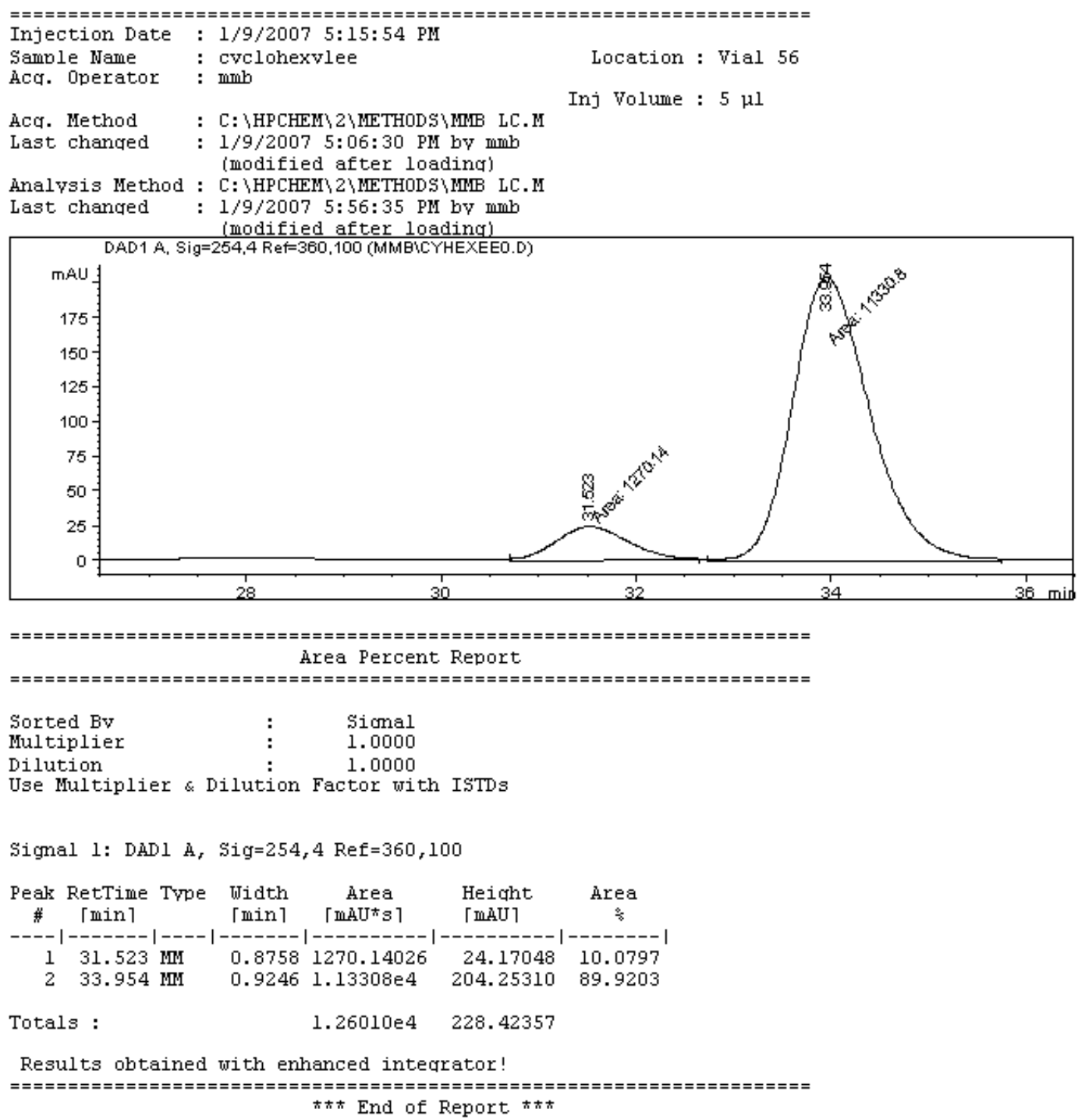




\title{
Racemic 2-Phenethylchroman-4-one (20):
}

HPLC Conditions: 95:5 hexanes:isopropanol, $1.0 \mathrm{~mL} / \mathrm{min}$, OD-H Chiralcel column

\author{
Data File C: $\backslash$ HPCHEM $\backslash 2 \backslash D A T A M \backslash M M \backslash M M E I-251 . D$
}

MMBI-250- racemic hYdrocinnamaldehYde- -C02

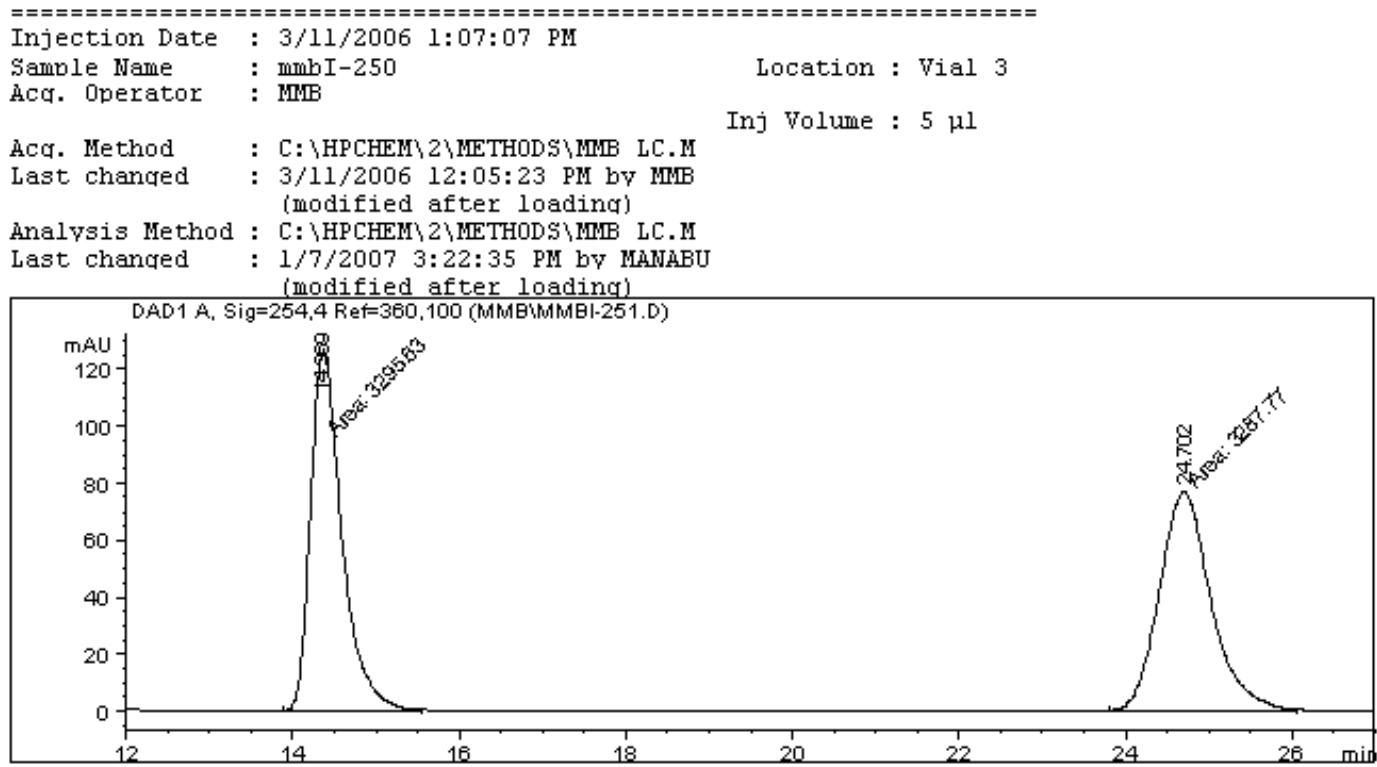

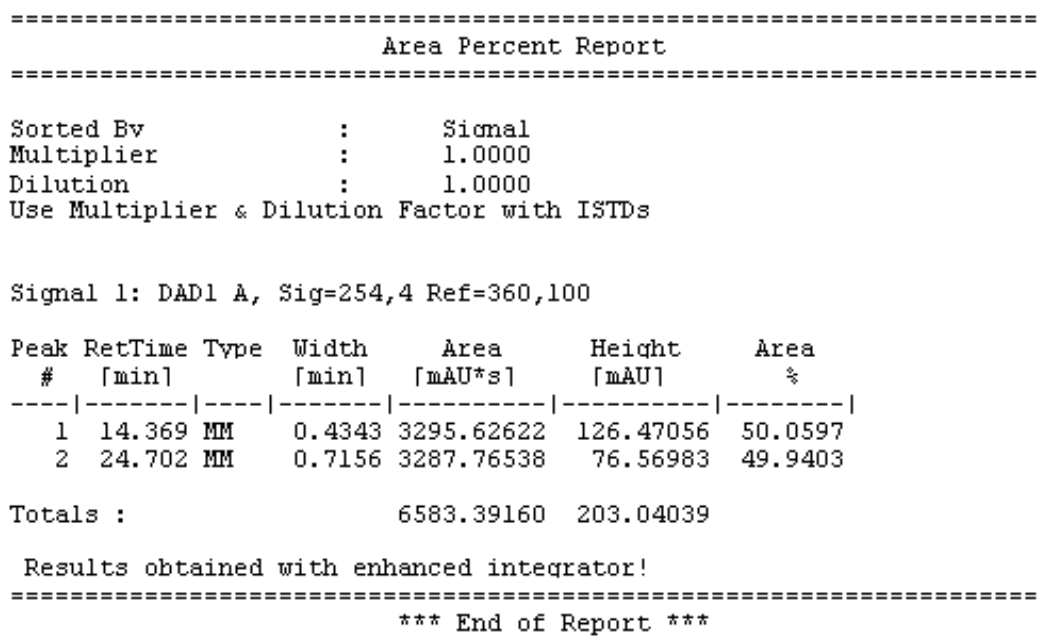


Enantioenriched $(\boldsymbol{R})$-2-Phenethylchroman-4-one (20):

HPLC Conditions: 95:5 hexanes:isopropanol, $1.0 \mathrm{~mL} / \mathrm{min}$, OD-H Chiralcel column Data File C: $\backslash$ HPCHEM $\backslash 2 \backslash D A T A M \backslash M B \backslash M M E I 2480 . D$

MMBI-248- Na2S04 hydrocinnamaldehyde- -C02

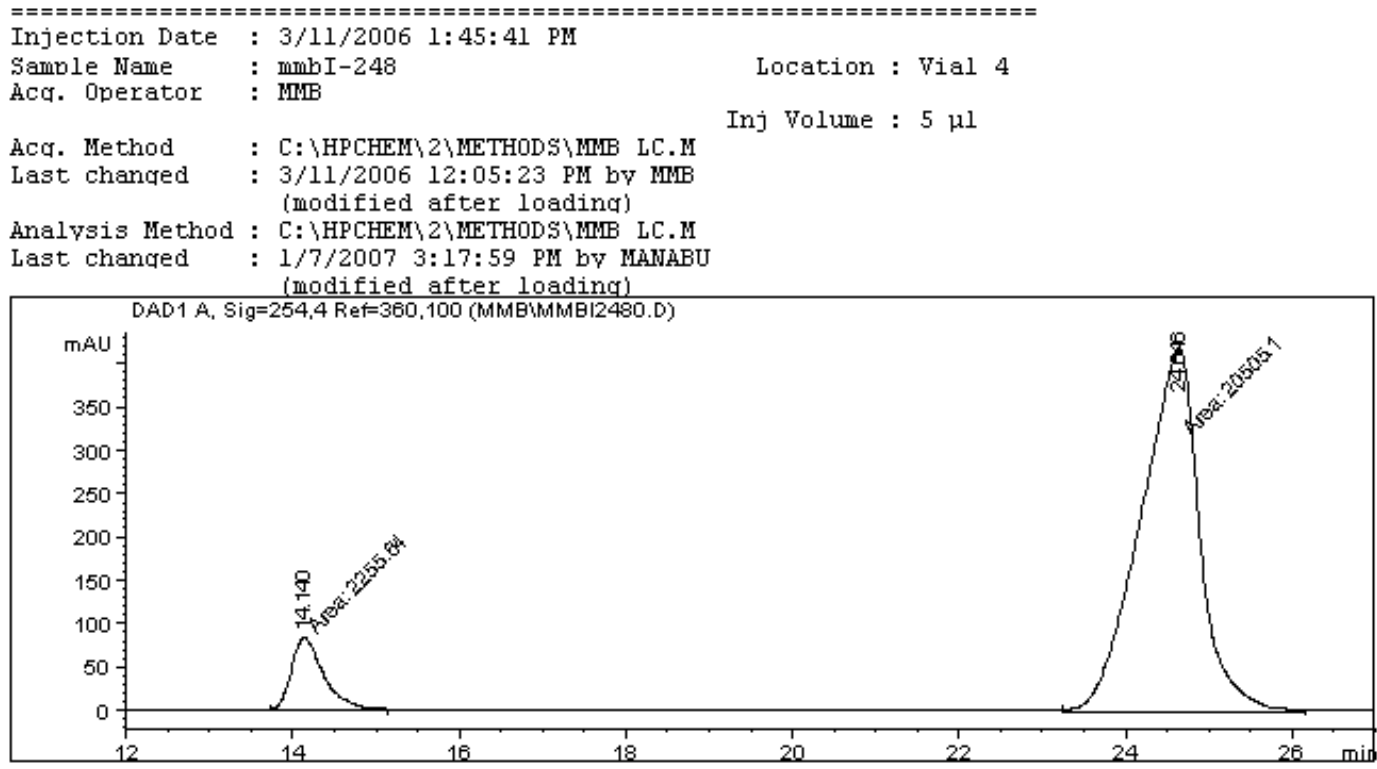

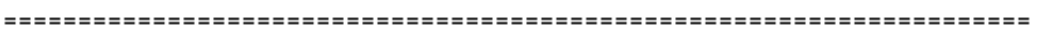

Area Percent Report

\begin{tabular}{|c|c|}
\hline Sorted Bv & : \\
\hline Multiplier & : \\
\hline Dilution & : \\
\hline
\end{tabular}

Use Multiplier \& Dilution Factor with ISTDs

Signal 1: DADl Aे, Sig=254, 4 Ref $=360,100$

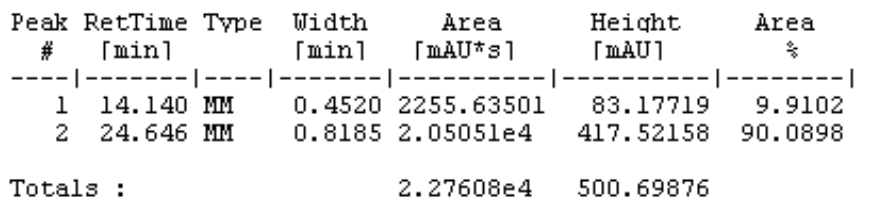

Results obtained with enhanced integrator!

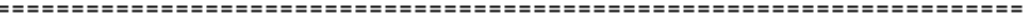

*ネ* End of Report 
X-ray Crystal Data for (E)-tert-butyl 3-(4-bromophenyl)-2-(2hydroxyphenylcarbonyl)prop-2-enoate:

X-ray diffraction was performed at $-120{ }^{\circ} \mathrm{C}$ and raw frame data were processed using SAINT. Molecular structure was solved using direct methods and refined by F2 by fullmatrix least-squares techniques. The GOF $=1.077$ for 233 variables refined to $\mathrm{R} 1=$ 0.0408 for 4435 reflections with $\mathrm{I}>2 \sigma(\mathrm{I})$. Further information is contained in the CIF file.

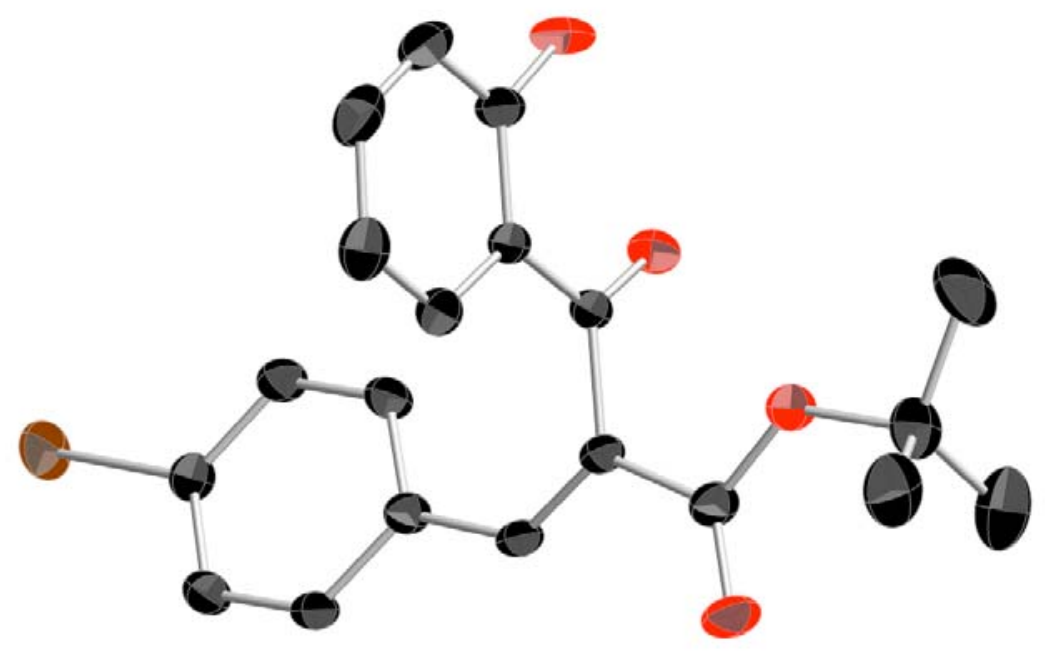

